Discrete Comput Geom 26:429-461 (2001)

DOI: $10.1007 / \mathrm{s} 00454-001-0008-0$

\title{
Equivelar Polyhedra with Few Vertices
}

\author{
B. Datta and N. Nilakantan \\ Department of Mathematics, Indian Institute of Science, \\ Bangalore 560 012, India \\ \{dattab,nandini\}@math.iisc.ernet.in
}

\begin{abstract}
We know that the polyhedra corresponding to the Platonic solids are equivelar. In this article we have classified completely all the simplicial equivelar polyhedra on $\leq 11$ vertices. There are exactly 27 such polyhedra. For each $n \geq-4$, we have classified all the $(p, q)$ such that there exists an equivelar polyhedron of type $\{p, q\}$ and of Euler characteristic $n$. We have also constructed five types of equivelar polyhedra of Euler characteristic $-2 m$, for each $m \geq 2$.
\end{abstract}

\section{Introduction}

A finite collection $K$ of cycles, edges and vertices of a complete graph is called a complex (of dimension 2) if (i) each edge of a cycle in $K$ is in $K$, (ii) each vertex of each edge in $K$ is in $K$ and (iii) any two cycles have at most one common edge. The cycles, edges and vertices in a complex are called the faces, edges and vertices in that complex, respectively. We denote a face $u_{1} \cdots u_{m} u_{1}$ by $u_{1} \cdots u_{m}$ also.

For a complex $K$, the edge graph $\mathrm{EG}(K)$ of $K$ is the graph whose vertices and edges are the vertices and edges of $K$, respectively. $\operatorname{EG}(K)$, is also called the 1-skeleton of $K$. The graph theoretic complement of $\operatorname{EG}(K)$ is called the non-edge graph of $K$ and is denoted by $\operatorname{NEG}(K)$. So, $e$ is an edge in $\operatorname{NEG}(K)$ if and only if $e$ is not an edge in $K$. See [2] for the graph-theoretic terms used in this paper.

If $K$ is a complex, then we associate another graph $\Lambda(K)$ with $K$ as follows. The vertices of $\Lambda(K)$ are the faces in $K$ and for faces $F_{1}, F_{2} \in K, F_{1} F_{2}$ is an edge in $\Lambda(K)$ whenever $F_{1}$ and $F_{2}$ have a common edge. For a vertex $u$ in $K$ let $\mathcal{F}_{u}$ be the set of faces containing $u$. A complex $K$ is called an abstract polyhedron (or simply a polyhedron) (of dimension 2) if (iv) for each vertex $v$ there is a face $F$ containing $v$, (v) each edge is in exactly two faces, (vi) the induced subgraph $L(u)=\Lambda(K)\left[\mathcal{F}_{u}\right]$ is a cycle for each vertex 
$u$ in $K$ and (vii) the graph $\Lambda(K)$ is connected. Since all the polyhedra considered in this paper are two-dimensional, we drop the qualification "two-dimensional". Clearly, the faces of a polyhedron determine the polyhedron. Because of this we identify a polyhedron with the set of faces in it.

A complex may be thought of as a prescription for the construction of a topological space by pasting together plane polygons. The topological space thus obtained from a complex $K$ is called the geometric carrier of $K$ and is denoted by $|K|$. It is easy to see that the geometric carrier of a polyhedron is a connected two-dimensional manifold.

Two complexes $K$ and $L$ are called isomorphic (denoted by $K \cong L$ ) if there exists a bijective map $\varphi$ from the vertex-set of $K$ to the vertex-set of $L$ such that $v_{1} \cdots v_{k}$ is a face in $K$ if and only if $\varphi\left(v_{1}\right) \cdots \varphi\left(v_{k}\right)$ is a face in $L$. We identify two complexes if they are isomorphic.

If $u v$ is an edge in a complex $K$, then we say $u$ and $v$ are adjacent in $K$. For a vertex $v$ in a complex $K$, the number of edges through $v$ is called the degree of $v$ in $K$. If $f_{0}(K), f_{1}(K)$ and $f_{2}(K)$ are the number of vertices, edges and faces, respectively, of a polyhedron $K$, then the number $\chi(K):=f_{0}(K)-f_{1}(K)+f_{2}(K)$ is called the Euler characteristic of $K$.

A polyhedron $K$ is called equivelar of type $\{p, q\}$ (or $\{p, q\}$-equivelar) if each face is a $p$-gon (i.e., $\Lambda(K)$ is a $p$-regular graph) and the degree of each vertex is $q$ (see [4]). A polyhedron is called equivelar if it is equivelar of type $\{p, q\}$ for some $p$ and $q$.

A complex is called simplicial if each face consists of three vertices. If $u$ is a vertex of a simplicial complex $K$, then the link of $u$ in $K$ (denoted by $\operatorname{Lk}_{K}(u)$ ) is the graph whose vertices are those vertices of $K$ which are adjacent to $u$ and whose edges are those edges $v w$ in $K$ such that $u v w$ is a face in $K$. A simplicial complex with properties (iv)-(vi) is called a combinatorial 2-manifold. Observe that in this case the link of any vertex is a cycle. So, a connected combinatorial 2-manifold in which the degree of each vertex is the same is a simplicial equivelar polyhedron and hence is called an equivelar combinatorial 2-manifold.

In [18]-[20] McMullen et al. considered equivelar polyhedra with geometric carriers in $\mathbb{R}^{3}$ (and hence orientable). We consider both the orientable and non-orientable cases.

Example 1. Some equivelar polyhedra:

$$
\begin{aligned}
S_{4}^{2}= & \{a b c, a b d, a c d, b c d\}, \\
O= & \left\{a_{i} b_{j} c_{k}: 1 \leq i, j, k \leq 2\right\}, \\
C= & \left\{a_{1} b_{2} c_{1} d_{2}, a_{1} b_{2} d_{1} c_{2}, a_{1} c_{2} b_{1} d_{2}, a_{2} b_{1} c_{2} d_{1}, a_{2} b_{1} d_{2} c_{1}, a_{2} c_{1} b_{2} d_{1}\right\}, \\
I= & \left\{u u_{i} u_{i+1}, u_{i} u_{i+1} v_{i+3}, v_{i} v_{i+1} u_{i+3}, v v_{i} v_{i+1}: 1 \leq i \leq 5\right\}, \\
D= & \left\{v_{1} v_{2} v_{3} v_{4} v_{5}, v_{i} v_{i+1} u_{i+1} v_{i, i+1} u_{i}, v_{i, i+1} u_{i+1} v_{i+1, i+2} u_{i+1, i+2} u_{i, i+1},\right. \\
& \left.u_{12} u_{23} u_{34} u_{45} u_{51}: 1 \leq i \leq 5\right\}, \\
\mathbb{R} P_{6}^{2}= & \left\{u u_{i} u_{i+1}, u_{i} u_{i+1} u_{i+3}: 1 \leq i \leq 5\right\}, \\
R= & \left\{u_{1,2} u_{2,3} u_{3,4} u_{4,5} u_{5,1}, u_{i, i+1} u_{i, i+1, i+3} u_{i+2, i+3, i} u_{i+5, i, i+2} u_{i+5, i}: 1 \leq i \leq 5\right\}, \\
M_{1}= & \left\{u_{1+p} u_{4+p} u_{7+p}, u_{i+3 p} u_{j+3 p} u_{k+3 p}:(i, j, k)\right. \\
& \in\{(1,2,5),(1,3,5),(1,3,4),(1,8,9),(1,6,8),(1,2,6),(2,3,6)\}, \\
& 0 \leq p \leq 2\},
\end{aligned}
$$




$$
N_{1}=\left\{u u_{i} u_{i+1}, u_{i} u_{i+1} u_{i+4}, u_{i} u_{i+2} u_{i+4}, u_{i} u_{i+3} u_{i+6}: 1 \leq i \leq 9\right\} .
$$

(Additions in the subscripts are modulo 5 in $I, D, \mathbb{R} P_{6}^{2}, R$ and are modulo 9 in $M_{1}, N_{1}$.)

Here $S_{4}^{2}$ is equivelar of type $\{3,3\}, O$ is equivelar of type $\{3,4\}, C$ is equivelar of type $\{4,3\}, I$ and $\mathbb{R} P_{6}^{2}$ are equivelar of type $\{3,5\}, D$ and $R$ are equivelar of type $\{5,3\}, M_{1}$ is equivelar of type $\{3,8\}$ and $N_{1}$ is equivelar of type $\{3,9\}$.

The geometric carrier of each of $S_{4}^{2}, O, C, I$ and $D$ is the 2-sphere and they correspond to the Platonic solids [5], [6], [12], [8], [24], namely, tetrahedron, octahedron, cube, icosahedron and dodecahedron, respectively. The polyhedron $\mathbb{R} P_{6}^{2}[1]$ is called the hemiicosahedron and the polyhedron $R$ is called the hemi-dodecahedron. The geometric carrier of each of $\mathbb{R} P_{6}^{2}$ and $R$ is the real projective plane. The geometric carrier of $M_{1}$ is the non-orientable surface of Euler characteristic -3 . The geometric carrier of $N_{1}$ is the non-orientable surface of Euler characteristic -5 .

Let $K$ be a polyhedron with faces $F_{1}, \ldots, F_{m}$. Consider a complex $\widetilde{K}$ with vertex-set $\left\{w_{1}, \ldots, w_{m}\right\}$ as: $w_{i_{1}} \cdots w_{i_{k}}$ is a face in $\widetilde{K}$ if and only if there exists a vertex $u$ in $K$ such that $F_{i_{1}} \cdots F_{i_{k}} F_{i_{1}}$ is the cycle $L(u)$ defined above. Then $\widetilde{K}$ is a polyhedron. $\widetilde{K}$ is called the dual of $K$. It is easy to show that the dual of $\widetilde{K}$ is isomorphic to $K$ and $\chi(\widetilde{K})=\chi(K)$. It is also not difficult to see that $\widetilde{S}_{4}^{2} \cong S_{4}^{2}, \widetilde{C} \cong O, \widetilde{I} \cong D$ and $\widetilde{\mathbb{R} P}_{6}^{2} \cong R$. Observe that the graph $\Lambda(K)$ is isomorphic to $\mathrm{EG}(\widetilde{K})$. Because of this, for a polyhedron $K, \Lambda(K)$ is called the dual 1-skeleton of $K$.

A pattern on a connected surface $M$ is a non-empty, connected locally finite graph $\Gamma$ contained in $M$, such that each component of $M \backslash \Gamma$ is simply connected and has compact closure. The closure of a component of $M \backslash \Gamma$ is called a face of $\Gamma$. A pattern decomposes the surface into faces. Such a decomposition is called a map (see [7], [15] and [16]). A pattern $\Gamma$ is called non-singular if each edge of $\Gamma$ lies in two faces. A pattern $\Gamma$ is called equivelar of type $\{p, q\}$ (or a $\{p, q\}$-pattern) if each face contains $p$ (counted with multiplicity) edges and each vertex has degree $q$.

Let $\Phi(n)=\{(p, q)$ : there exists a $\{p, q\}$-equivelar polyhedron of Euler characteristic $n\}$ and $\Sigma(n)$ denote the set of all equivelar polyhedra of Euler characteristics $n$. Clearly, $\Phi(m)=\emptyset$ for $m \geq 3$. It is known (e.g., see [17]) that if $(p, q) \in \Phi(n)$ for some $n<0$, then $(p, q) \in \Phi(n)$ for infinitely many negative $n$. Here we prove:

Theorem 1. If $\Phi(n)$ and $\Sigma(n)$ are as above, then

(i) $\Phi(n) \cap \Phi(-m)=\emptyset$ for all $n \geq 0$ and $m \geq 1$,

(ii) $\Phi(2)=\{(3,3),(3,4),(4,3),(3,5),(5,3)\}$,

(iii) $\Phi(1)=\{(3,5),(5,3)\}$,

(iv) $\Phi(0)=\{(3,6),(6,3),(4,4)\}$,

(v) $\Phi(-1)=\emptyset$,

(vi) $\Phi(-2)=\{(3,7),(7,3),(4,5),(5,4)\}$,

(vii) $\Phi(-3)=\{(3,7),(7,3),(3,8),(8,3),(4,5),(5,4),(5,5)\}$,

(viii) $\Phi(-4)=\{(3,7),(7,3),(3,8),(8,3),(4,5),(5,4),(4,6),(6,4),(5,5)\} \subseteq$ $\Phi(-2 m)$, for all $m \geq 3$,

(ix) $(3,3 k-1) \in \Phi(-k(3 k-7) / 2),(3,3 k) \in \Phi(1-k(3 k-5) / 2)$, for all $k \geq 3$, 
(x) $\Phi(n)$ is a finite set for each integer $n$ and

(xi) for each $n \geq 7$, there exists an $n$-vertex $\{3,6\}$-equivelar polyhedron in $\Sigma(0)$.

Corollary 2. For each $n \neq 0$, there exist only finitely many equivelar polyhedra of Euler characteristic $n$.

In [11] (also see in [7]) Edmonds et al. proved the existence and uniqueness of a $\{p, q\}$-pattern on surfaces. Clearly, a $\{p, q\}$-equivelar polyhedron $K$ gives a non-singular $\{p, q\}$-pattern on $|K|$ with the property that any two faces have at most one common edge. So, the existence of a $\{p, q\}$-equivelar polyhedron implies the existence of a $\{p, q\}$ pattern but not conversely. For example, by Theorem 2.4(i) of [11], a \{3,7\}-pattern and a $\{4,6\}$-pattern exist on a non-orientable surface of Euler characteristic -1 but, from Theorem 1(v) above, an equivelar polyhedron of Euler characteristic -1 does not exist. Also, by the same theorem in [11], a $\{5,6\}$-pattern exists on an orientable surface of Euler characteristic -4 but, from Theorem 1 (viii) above a $\{5,6\}$-equivelar polyhedron of Euler characteristic -4 does not exist. There are five choices of $(p, q)$ for $\{p, q\}$-equivelar polyhedra of Euler characteristic 2, where as there are infinitely many choices of $(p, q)$ for $\{p, q\}$-patterns (e.g., $\{p, 2\}$-patterns exist for all $p \geq 3$ ) on the 2-sphere (see [11] and [7]). However, in each case unique pattern exists (see Classification in [11]). Similarly for Euler characteristic 1. From these (Classification in [11]) and Theorem 1(iii) and (iv) above we get:

Corollary 3. If the Euler characteristic of an equivelar polyhedron is positive, then the polyhedron is $S_{4}^{2}, C, O, I, D, \mathbb{R} P_{6}^{2}$ or $R$ defined in Example 1.

Corollary 2 says that $\Sigma(n)$ is a finite set for $n \neq 0$ and Theorem 1(xi) shows that $\Sigma(0)$ is an infinite set. If the Euler characteristic is $\leq 0$, then (unlike when the Euler characteristic is $>0$ ) it is in general difficult to classify all the non-singular $\{p, q\}$ patterns. In particular, it is very difficult to classify all the $\{p, q\}$-equivelar polyhedra of a given non-positive Euler characteristic. Even for a negative Euler characteristic, there can exist more than one $\{p, q\}$-equivelar polyhedra of the same Euler characteristic (e.g., $N_{1}, \ldots, N_{14}$ in Examples 1 and 8). For simplicial polyhedra on few vertices we have:

Theorem 4. Let $K$ be an n-vertex simplicial equivelar polyhedron of Euler characteristic 0 . If $n \leq 11$, then $K$ is isomorphic to $T_{7}, \ldots, T_{11}, A_{3,3}, B_{3,3}$ or $Q$ defined below.

Theorem 5. If $M_{1}$ and $M_{2}$ are as in Examples 1 and 7 , then $M_{1} ¥ M_{2}$ and any 9-vertex neighbourly simplicial equivelar polyhedron is isomorphic to $M_{1}$ or $M_{2}$.

Theorem 6. Let $N_{1}, \ldots, N_{14}$ be as in Examples 1 and 8. We have the following:

(i) $N_{i}$ is not isomorphic to $N_{j}$ for $1 \leq i \neq j \leq 14$.

(ii) If $M$ is a 10-vertex neighbourly simplicial equivelar polyhedron, then $M$ is isomorphic to $N_{i}$ for some $i \in\{1, \ldots, 14\}$. 
Theorem 7. There are exactly 27 (up to isomorphism) simplicial equivelar polyhedra on $\leq 11$ vertices, namely, $S_{4}^{2}, O, \mathbb{R} P_{6}^{2}, A_{3,3}, B_{3,3}, T_{7}, \ldots, T_{11}, M_{1}, M_{2}, N_{1}, \ldots, N_{14}$ and $Q$, defined in Examples 1-8.

Remark 1. Observe that $M_{1}$ and $M_{2}$ are non-isomorphic but they have the same 1skeleton. Similarly, $N_{1}, \ldots, N_{14}$ have the same 1-skeleton but they are pairwise nonisomorphic combinatorial 2-manifolds.

Remark 2. Corollary 3 is classically known. We have added it here as an immediate consequence of Theorem 1. Corollary 2 is also known (e.g., see [25]). We have added it for the sake of completeness.

Remark 3. In this article we consider polyhedra from a combinatorial point of view. For some polyhedra (e.g., $L_{2}, L_{3}, Q, G, D_{n}$ 's, $H_{n}$ 's, . .) we have given their geometric realizations in Section 2. A polyhedron $K$ is also called a polyhedral 2-manifold (see [4]).

Remark 4. Property (vii), in the definition of a polyhedron, implies that the geometric carrier of a polyhedron is connected. A complex with properties (iv)-(vi) is said to be a weak polyhedron. Similarly, we can define $\{p, q\}$-equivelar and equivelar weak polyhedra. Clearly, an equivelar weak polyhedron is the disjoint union of equivelar polyhedra whose Euler characteristic is the sum of the Euler characteristics of the components. So, it is sufficient to consider only equivelar polyhedra.

\section{Examples}

In this section we construct infinitely many equivelar polyhedra. Some of them have already been mentioned in the previous section. We use others in the next section. Recall that we identify a polyhedron with the set of faces in it. At the end of this section we give the geometric realizations of some of the polyhedra.

Example 2. Some equivelar polyhedra of Euler characteristic 0:

$$
\begin{aligned}
A_{m, n}= & \left\{u_{i, j} u_{i+1, j} u_{i+1, j+1}, u_{i, j} u_{i, j+1} u_{i+1, j+1}: 1 \leq i \leq m, 1 \leq j \leq n\right\}, \quad m, n \geq 3 . \\
B_{m, n}= & \left\{u_{i, j} u_{i+1, j} u_{i+1, j+1}, u_{i, j} u_{i, j+1} u_{i+1, j+1}: 1 \leq i \leq m-1,1 \leq j \leq n\right\} \\
& \cup\left\{u_{m, j} u_{1, n+2-j} u_{1, n+1-j}, u_{m, j} u_{m, j+1} u_{1, n+1-j}: 1 \leq j \leq n\right\}, \quad m, n \geq 3 . \\
C_{m, n}= & \left\{u_{i, j} u_{i+1, j} u_{i+1, j+1} u_{i, j+1}: 1 \leq i \leq m, 1 \leq j \leq n\right\}, \quad m, n \geq 3 .
\end{aligned}
$$

(Additions in the first and second subscripts are modulo $m$ and $n$, respectively.)

$$
\begin{aligned}
& T_{n}=\left\{u_{i} u_{i+1} u_{i+3}, u_{i} u_{i+2} u_{i+3}: 1 \leq i \leq n\right\}, \quad n \geq 7 . \\
& \quad(\text { Additions in the subscripts are modulo } n .) \\
& Q=\{012,023,034,045,056,016,127,136,138,178,236,269,279, \\
&348,457,479,489,569,578,589\} .
\end{aligned}
$$


$A_{m, n}, B_{m, n}, T_{n}$ and $Q$ are equivelar of type $\{3,6\}$ and $C_{m, n}$ is equivelar of type $\{4,4\}$. The geometric carriers of $A_{m, n}, C_{m, n}$ and $T_{n}$ are the torus and the geometric carriers of $B_{m, n}$ and $Q$ are the Klein bottle.

Example 3. Some equivelar polyhedra of type $\{4,5\}$ :

$$
\begin{aligned}
& D_{n}= {\left[\left\{a_{i, j, k} a_{i, j+1, k} a_{i, j+1, k+1} a_{i, j, k+1}: 1 \leq j, k \leq 4,1 \leq i \leq n\right\} \backslash\right.} \\
&\left\{a_{i, j, k} a_{i, j+1, k} a_{i, j+1, k+1} a_{i, j, k+1}:(j, k)\right. \\
& \in\{(1,1),(1,3),(3,1),(3,3)\}, 1 \leq i \leq n\}] \\
& \cup\left\{a_{i, 3, k} a_{i, 3, k+1} a_{i+1,2, k+1} a_{i+1,2, k}, a_{i, 3, k+1} a_{i, 4, k+1} a_{i+1,1, k+1} a_{i+1,2, k+1},\right. \\
& a_{i, 4, k+1} a_{i, 4, k} a_{i+1,1, k} a_{i+1,1, k+1}, a_{i, 4, k} a_{i, 3, k} a_{i+1,2, k} a_{i+1,1, k}: \\
&k \in\{1,3\}, 1 \leq i \leq n\}, \quad \text { for } \quad n \geq 2 . \\
& E_{n}=\left[\left\{a_{i, j, k} a_{i, j+1, k} a_{i, j+1, k+1} a_{i, j, k+1}: 1 \leq j, k \leq 4, \quad 1 \leq i \leq n\right\} \backslash\right. \\
&\left\{a_{i, j, k} a_{i, j+1, k} a_{i, j+1, k+1} a_{i, j, k+1}:(j, k)\right. \\
&\in\{(1,1),(1,3),(3,2),(3,4)\}, 1 \leq i \leq n\}] \\
& \cup\left\{a_{i, 3, k} a_{i, 3, k+1} a_{i+1,2, k} a_{i+1,2, k+3}, a_{i, 3, k+1} a_{i, 4, k+1} a_{i+1,1, k} a_{i+1,2, k},\right. \\
& a_{i, 4, k+1} a_{i, 4, k} a_{i+1,1, k+3} a_{i+1,1, k}, a_{i, 4, k} a_{i, 3, k} a_{i+1,2, k+3} a_{i+1,1, k+3}: \\
&k \in\{2,4\}, 1 \leq i \leq n\} . \quad \text { for } n \geq 1 .
\end{aligned}
$$

(Additions in the first subscripts are modulo $n$ and in the second and third subscripts are modulo 4.)

$$
\begin{aligned}
F_{1}= & \left\{a_{i} c_{i} d_{i+1} d_{i}, a_{i+1} a_{i} d_{i} b_{i+1}, a_{i} b_{i} b_{i+1} c_{i+1}, b_{i} d_{i} c_{i+1} c_{i}, a_{1} c_{1} a_{2} c_{2}, b_{1} d_{1} b_{2} d_{2}:\right. \\
& 1 \leq i \leq 2\} .
\end{aligned}
$$

(Additions in the subscripts are modulo 2.)

$$
\begin{aligned}
F_{n}= & {\left[\left\{a_{i, j} a_{i, j+1} a_{i+1, j+1} a_{i+1, j}: 1 \leq j \leq 4,1 \leq i \leq 2 n\right\} \backslash\right.} \\
& \left.\left\{a_{2 k-1, j} a_{2 k-1, j+1} a_{2 k, j+1} a_{2 k, j}: j \in\{1,3\}, 1 \leq k \leq n\right\}\right] \\
\cup & \left\{a_{2 k-1,1} a_{2 k-1,2} a_{2 k+2,4} a_{2 k+2,3}, a_{2 k-1,2} a_{2 k, 2} a_{2 k+1,4} a_{2 k+2,4},\right. \\
& a_{2 k, 2} a_{2 k, 1} a_{2 k+1,3} a_{2 k+1,4}, a_{2 k, 1} a_{2 k-1,1} a_{2 k+2,3} a_{2 k+1,3}: \\
& 1 \leq k \leq n\}, \quad \text { for } \quad n \geq 2 . \\
G_{n}=[ & \left\{a_{i, j} a_{i, j+1} a_{i+1, j+1} a_{i+1, j}: 1 \leq j \leq 4,1 \leq i \leq 2 n\right\} \backslash \\
& \left.\left\{a_{2 k-1, j} a_{2 k-1, j+1} a_{2 k, j+1} a_{2 k, j}: j \in\{1,3\}, 1 \leq k \leq n\right\}\right] \\
\cup & \left\{a_{2 k-1,1} a_{2 k-1,2} a_{2 k+1,4} a_{2 k+1,3}, a_{2 k-1,2} a_{2 k, 2} a_{2 k+2,4} a_{2 k+1,4},\right. \\
& a_{2 k, 2} a_{2 k, 1} a_{2 k+2,3} a_{2 k+2,4}, a_{2 k, 1} a_{2 k-1,1} a_{2 k+1,3} a_{2 k+2,3}: \\
& 1 \leq k \leq n\}, \quad \text { for } n \geq 2 .
\end{aligned}
$$

(Additions in the first and second subscripts are modulo $2 n$ and 4 , respectively.)

$G=\{a b c d$, adef, bagh, cbij, dckl, feij, hgkl, aljg, alhf, bkeh, bkgi, ciek, cigj, dfhe, $d f j l\}$. 
$\chi\left(D_{n}\right)=16 n-40 n+20 n=-4 n$ and the geometric carrier of $D_{n}$ is the orientable surface of genus $2 n+1$ for all $n \geq 2$. $\chi\left(E_{n}\right)=-4 n$ and the geometric carrier of $E_{n}$ is the orientable surface of genus $2 n+1$ for all $n \geq 1$. $\chi\left(F_{n}\right)=8 n-20 n+10 n=-2 n$ and the geometric carrier of $F_{n}$ is the orientable surface of genus $n+1$ for all $n \geq 1$. $\chi\left(G_{n}\right)=-2 n$ and the geometric carrier of $G_{n}$ is a non-orientable surface for all $n \geq 2$. The geometric carrier of $G$ is the non-orientable surface of Euler characteristic -3 .

Example 4. Some equivelar polyhedra of type $\{3,7\}$ :

$$
\begin{aligned}
& H_{n}=\left\{u_{i, 1} v_{i, 1} u_{i, 2}, u_{i, 2} v_{i, 1} v_{i, 2}, u_{i, 2} v_{i, 2} v_{i, 3}, u_{i, 2} v_{i, 3} u_{i, 3}, u_{i, 4} v_{i, 4} u_{i, 1}, u_{i, 1} v_{i, 4} v_{i, 1}\right. \text {, } \\
& v_{i, 2} w_{i, 2} w_{i, 3}, v_{i, 2} w_{i, 3} v_{i, 3}, v_{i, 3} w_{i, 3} v_{i, 4}, v_{i, 4} w_{i, 3} w_{i, 4}, v_{i, 4} w_{i, 4} v_{i, 1}, v_{i, 1} w_{i, 4} w_{i, 1} \text {, } \\
& w_{i, 1} u_{i, 1} w_{i, 2}, w_{i, 2} u_{i, 1} u_{i, 2}, w_{i, 2} u_{i, 2} w_{i, 3}, w_{i, 3} u_{i, 2} u_{i, 3}, w_{i, 3} u_{i, 3} w_{i, 4}, w_{i, 4} u_{i, 3} u_{i, 4}, \\
& \left.w_{i, 4} u_{i, 4} u_{i, 1}, w_{i, 4} u_{i, 1} w_{i, 1}\right\} \\
& \cup\left\{u_{i+1,3} v_{i+1,3} w_{i, 2}, u_{i+1,3} w_{i, 2} v_{i, 2}, u_{i+1,3} u_{i+1,4} v_{i, 2}, u_{i+1,4} v_{i, 2} v_{i, 1},\right. \\
& u_{i+1,4} v_{i+1,4} w_{i, 1}, u_{i+1,4} v_{i, 1} w_{i, 1}, v_{i+1,4} v_{i+1,3} w_{i, 1}, v_{i+1,3} w_{i, 1} w_{i, 2} \text { : } \\
& 1 \leq i \leq n\}, \quad \text { for } n \geq 1 \text {. }
\end{aligned}
$$

(Additions in the subscripts are modulo $n$.)

$H=\left\{a_{1} b_{4} b_{1}, a_{1} b_{1} b_{3}, a_{1} b_{3} a_{2}, a_{1} a_{2} c_{2}, a_{1} c_{2} c_{1}, a_{2} b_{3} b_{2}, a_{2} b_{2} c_{5}, a_{2} c_{5} c_{4}, a_{2} c_{4} a_{3}, a_{2} a_{3} c_{2}\right.$, $a_{3} c_{4} b_{5}, a_{3} b_{5} b_{6}, a_{3} b_{6} a_{4}, a_{3} a_{4} c_{3}, a_{3} c_{3} c_{2}, a_{4} b_{6} b_{4}, a_{4} b_{4} b_{1}, a_{4} b_{1} a_{5}, a_{4} a_{5} c_{1}, a_{4} c_{1} c_{3}$, $a_{5} b_{1} b_{2}, a_{5} b_{2} c_{5}, a_{5} c_{5} c_{6}, a_{5} c_{6} a_{6}, a_{5} a_{6} c_{1}, a_{6} c_{6} c_{4}, a_{6} c_{4} b_{5}, a_{6} b_{5} b_{4}, a_{6} b_{4} a_{1}, a_{6} a_{1} c_{1}$, $b_{1} b_{2} b_{6}, b_{1} b_{3} b_{6}, b_{2} b_{3} b_{5}, b_{2} b_{5} b_{6}, b_{3} b_{4} b_{5}, b_{3} b_{4} b_{6}, c_{1} c_{2} c_{6}, c_{1} c_{3} c_{6}, c_{2} c_{3} c_{5}, c_{2} c_{5} c_{6}$, $\left.c_{3} c_{4} c_{5}, c_{3} c_{4} c_{6}\right\}$.

The geometric carrier of $H$ is the non-orientable surface of Euler characteristic -3 . The geometric carrier of $H_{n}$ is an orientable surface and $\chi\left(H_{n}\right)=12 n-42 n+28 n=-2 n$ for all $n \geq 1$.

Example 5. Some sequences of equivelar polyhedra of type $\{3,8\}$ :

$$
\begin{aligned}
& J_{n}=\left\{u_{i, j} u_{i+1, j} u_{i+1, j+1}, u_{i, j} u_{i, j+1} u_{i+1, j+1}: 1 \leq i \leq 2 n, 1 \leq j \leq 3\right\} \backslash \\
& \left\{u_{2 k-1, j} u_{2 k-1, j+1} u_{2 k, j+1}: 1 \leq k \leq n, j \in\{1,3\}\right\} \\
& \cup\left\{u_{2 k-1,1} u_{2 k+1,3} u_{2 k-1,2}, u_{2 k-1,2} u_{2 k+1,3} u_{2 k+2,3}, u_{2 k-1,2} u_{2 k+2,3} u_{2 k, 2},\right. \\
& u_{2 k, 2} u_{2 k+2,3} u_{2 k+2,1}, u_{2 k, 2} u_{2 k+2,1} u_{2 k-1,1}, u_{2 k-1,1} u_{2 k+2,1} u_{2 k+1,3} \text { : } \\
& 1 \leq k \leq n\}, \quad \text { for } n \geq 3 \text {. } \\
& K_{n}=\left[\left\{u_{i, j, k} u_{i, j+1, k} u_{i, j+1, k+1}, u_{i, j, k} u_{i, j, k+1} u_{i, j+1, k+1}, u_{i, l, k} v_{i, l+1, k} u_{i, l, k+1},\right.\right. \\
& \left.u_{i, l, k+1} u_{i, l+1, k} u_{i, l+1, k+1}: 1 \leq j \leq 2,3 \leq l \leq 4,1 \leq k \leq 3,1 \leq i \leq n\right\} \backslash \\
& \left.\left\{u_{i, 1,1} u_{i, 2,2} u_{i, 1,2}, u_{i, 1,3} u_{i, 2,3} u_{i, 2,1}, u_{i, 3,2} u_{i, 4,1} u_{i, 4,2}, u_{i, 3,3} u_{i, 4,3} u_{i, 3,1}\right\}\right] \\
& \cup\left\{u_{i, 1,1} u_{i, 4,3} u_{i, 3,3}, u_{i, 1,1} u_{i, 3,3} u_{i, 1,2}, u_{i, 1,2} u_{i, 3,3} u_{i, 3,1}, u_{i, 1,2} u_{i, 3,1} u_{i, 2,2}\right. \text {, } \\
& u_{i, 2,2} u_{i, 3,1} u_{i, 4,3}, u_{i, 2,2} u_{i, 4,3} u_{i, 1,1}, u_{i, 4,1} u_{i+1,2,1} u_{i+1,2,3},
\end{aligned}
$$




$$
\begin{aligned}
& u_{i, 4,1} u_{i+1,2,3} u_{i, 3,2}, \quad u_{i, 3,2} u_{i+1,2,3} u_{i+1,1,3}, \quad u_{i, 3,2} u_{i+1,1,3} u_{i, 4,2}, \\
& \left.u_{i, 4,2} u_{i+1,1,3} u_{i+1,2,1}, \quad u_{i, 4,2} u_{i+1,2,1} u_{i, 4,1}: 1 \leq i \leq n\right\}, \quad \text { for } n \geq 1 .
\end{aligned}
$$

(Additions in the first and second subscripts are modulo $n$ and 3 , respectively.)

$\chi\left(J_{n}\right)=6 n-24 n+16 n=-2 n$ and the geometric carrier of $J_{n}$ is the orientable surface of genus $n+1$ for all $n \geq 3$. $\chi\left(K_{n}\right)=12 n-48 n+32 n=-4 n$ and the geometric carrier of $K_{n}$ is a non-orientable surface for all $n \geq 1$.

Example 6. Some sequences of equivelar polyhedra of type $\{4,6\}$ :

$$
\begin{aligned}
L_{2}= & \{1263,1374,1425,1536,1647,1752,8273,8346,8657,8724,8435,8562\} . \\
L_{n}= & \left\{a_{i, 1} a_{i+1,1} a_{i+1,2} a_{i, 2}, a_{i, 2} a_{i+1,2} b_{i+1,3} b_{i, 3}, b_{i, 3} b_{i+1,3} b_{i+1,4} b_{i, 4}, b_{i, 4} a_{i+1,4} a_{i+1,1} a_{i, 1}:\right. \\
& 1 \leq i \leq 2 n\} \backslash\left\{a_{2 k-1,1} a_{2 k, 1} a_{2 k, 2} a_{2 k-1,2}, b_{2 k-1,3} b_{2 k, 3} b_{2 k, 4} b_{2 k-1,4}: 1 \leq k \leq n\right\}, \\
& \text { where } \quad a_{2 k-1,1}=b_{2 k+1,4}, \quad a_{2 k-1,2}=b_{2 k+2,4}, \quad a_{2 k, 2}=b_{2 k+2,3}, \\
& a_{2 k, 1}=b_{2 k+1,3}, \\
& \text { for } n \geq 3 .
\end{aligned}
$$

(Additions in the subscripts are modulo $2 n$.)

$$
\begin{aligned}
P_{n}= & \left\{a_{i, j, k} a_{i, j+1, k} a_{i, j+1, k+1} a_{i, j, k+1}: 1 \leq j, k \leq 4,1 \leq i \leq n\right\} \backslash \\
& \left\{a_{i, j, k} a_{i, j+1, k} a_{i, j+1, k+1} a_{i, j, k+1}:(j, k)\right. \\
& \in\{(1,1),(1,3),(3,1),(3,3)\}, 1 \leq i \leq n\},
\end{aligned}
$$

where $\quad a_{i, j, k}=a_{i+1, j+2, k}$,

for $(j, k) \in\{(1,1),(1,2),(2,1),(2,2),(3,3),(3,4),(4,3),(4,4)\}$

and $1 \leq i \leq n$ for $n \geq 3$.

(Additions in the first subscripts are modulo $n$ and in the second and third subscripts are modulo 4.)

$\chi\left(L_{n}\right)=4 n-12 n+6 n=-2 n$ for all $n \geq 2$. The geometric carrier of $L_{2}$ is the orientable surface of genus 3 and the geometric carrier of $L_{n}$ is a non-orientable surface for all $n \geq 3$. $\chi\left(P_{n}\right)=8 n-24 n+12 n=-4 n$ and the geometric carrier of $P_{n}$ is the orientable surface of genus $2 n+1$ for all $n \geq 3$. (Note that $L_{n}$, for $n \geq 3$, is obtained from an $8 n$-vertex $\left(\left\{a_{i, j}, b_{i, k}: 1 \leq i \leq 2 n, 1 \leq j \leq 2,3 \leq k \leq 4\right\}\right)$ complex (torus with $2 n$ holes) by identifying $b_{l, k}$ 's with $a_{i, j}$ 's. $P_{n}$ is also obtained by some identifications.)

Example 7. Another 9-vertex neighbourly simplicial equivelar polyhedra:

$$
\begin{aligned}
M_{2}= & \{129,239, \ldots, 789,189,124,136,138,147,156,157, \\
& 237,245,258,267,268,346,357,358,468,478\},
\end{aligned}
$$

where the vertex set of $M_{2}$ is $\{0,1, \ldots, 8\}$. Clearly, $\chi\left(M_{2}\right)=-3$.

Example 8. Thirteen more 10-vertex neighbourly simplicial equivelar polyhedra:

$$
\begin{gathered}
N_{2}=A \cup\{134,136,156,158,178,179,237,248,257,259,268, \\
269,358,359,368,379,457,467,469,489\},
\end{gathered}
$$




$$
\begin{aligned}
& N_{3}=A \cup\{137,139,146,156,158,178,237,248,257,259,268 \text {, } \\
& 269,346,358,359,368,457,479,489,679\} \text {, } \\
& N_{4}=A \cup\{136,137,145,158,168,179,238,249,256,257,269 \text {, } \\
& 278,347,358,359,369,467,468,489,579\} \text {, } \\
& N_{5}=A \cup\{134,136,157,158,168,179,237,248,256,259,268 \text {, } \\
& 279,358,359,369,378,457,467,469,489\} \text {, } \\
& N_{6}=A \cup\{134,136,157,158,168,179,238,247,256,259,268 \text {, } \\
& 279,357,359,369,378,458,467,469,489\} \text {, } \\
& N_{7}=A \cup\{134,138,156,157,168,179,236,247,258,259,269 \text {, } \\
& 278,357,359,367,389,458,468,469,479\} \text {, } \\
& N_{8}=A \cup\{134,138,156,157,168,179,236,247,257,258,269 \text {, } \\
& 289,358,359,367,379,459,468,469,478\} \text {, } \\
& N_{9}=A \cup\{138,139,146,157,158,167,236,245,258,269,278 \text {, } \\
& 279,347,357,359,368,468,479,489,569\} \text {, } \\
& N_{10}=A \cup\{136,138,145,158,167,179,238,249,256,257,268 \text {, } \\
& 279,347,357,359,369,468,469,478,589\} \text {, } \\
& N_{11}=A \cup\{134,138,156,157,168,179,237,246,257,259,268 \text {, } \\
& 289,358,359,367,369,458,469,478,479\} \text {, } \\
& N_{12}=A \cup\{134,138,156,157,168,179,236,247,258,259,268 \text {, } \\
& 279,357,359,369,378,458,467,469,489\} \text {, } \\
& N_{13}=A \cup\{136,137,145,158,168,179,238,246,257,259,267 \text {, } \\
& 289,349,357,358,369,468,478,479,569\} \text {, } \\
& N_{14}=A \cup\{134,138,156,157,168,179,237,246,257,258,269 \text {, } \\
& 289,358,359,367,369,459,468,478,479\} \text {, }
\end{aligned}
$$

where the vertex set of $N_{i}(2 \leq i \leq 14)$ is $\{0,1, \ldots, 9\}$ and $A=\{012, \ldots, 089,019$, 124\}. Clearly, $\chi\left(N_{i}\right)=-5$ for $1 \leq i \leq 14$. Thus, all of them triangulate the same non-orientable surface of Euler characteristic -5 .

Ringel and Jungerman [13], [21]-[23], [14] have shown that there exist neighbourly simplicial polyhedra on $3 k$ and $3 k+1$ vertices, for each $k \geq 3$, i.e.,

Proposition 1. For $k \geq 2$, if $n=3 k$ or $3 k+1$, then there exists an $n$-vertex equivelar polyhedron of type $\{3, n-1\}$.

Thus, if $m=-k(3 k-7) / 2$ or $1-k(3 k-5) / 2$, for $k \geq 3$, then there exists an equivelar polyhedron of type $\left\{3, f_{0}-1\right\}$ of Euler characteristic $m$. In particular, there exist neighbourly equivelar polyhedra on nine and ten vertices $\left(M_{1}\right.$ and $N_{1}$, respectively, in Example 1). 


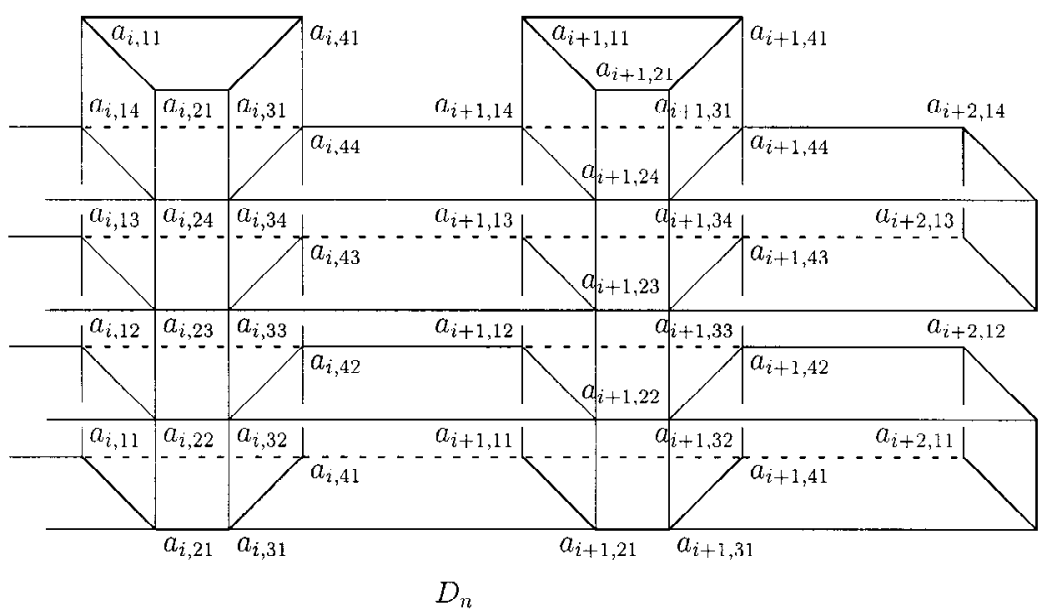

\begin{tabular}{|c|c|c|c|c|c|}
\hline $\begin{array}{l}a_{11} \\
a_{14}\end{array}$ & $a_{21}$ & $\begin{array}{l}a_{3 \mathrm{~J}} \\
a_{34} \\
\end{array}$ & $a_{41}$ & $\begin{array}{l}a_{51} \\
a_{54}\end{array}$ & $a_{61}$ \\
\hline$x$ & $a_{24}$ & $x$ & $a_{44}$ & $x$ & $a_{64}$ \\
\hline $\begin{array}{l}a_{13} \\
a_{12}\end{array}$ & $a_{23}$ & $\begin{array}{l}a_{33} \\
a_{32} \\
\end{array}$ & $a_{43}$ & $\begin{array}{l}a_{53} \\
a_{52}\end{array}$ & $a_{63}$ \\
\hline$x$ & $\overline{a_{22}}$ & $\times$ & $a_{42}$ & $x$ & $a_{62}$ \\
\hline$a_{11}$ & $a_{21}$ & $a_{31}$ & $a_{11}$ & $a_{5} \mathrm{l}$ & $a, 61$ \\
\hline
\end{tabular}
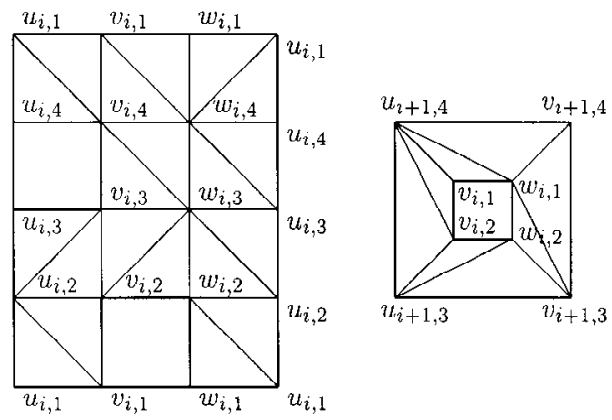

$H_{n}$
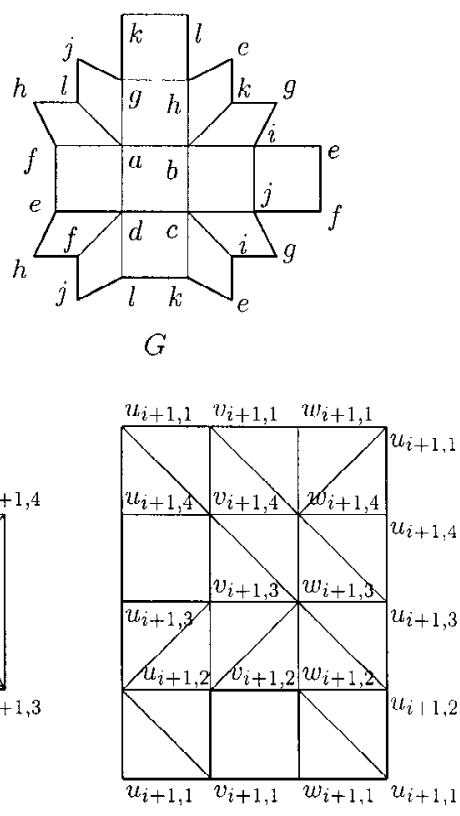

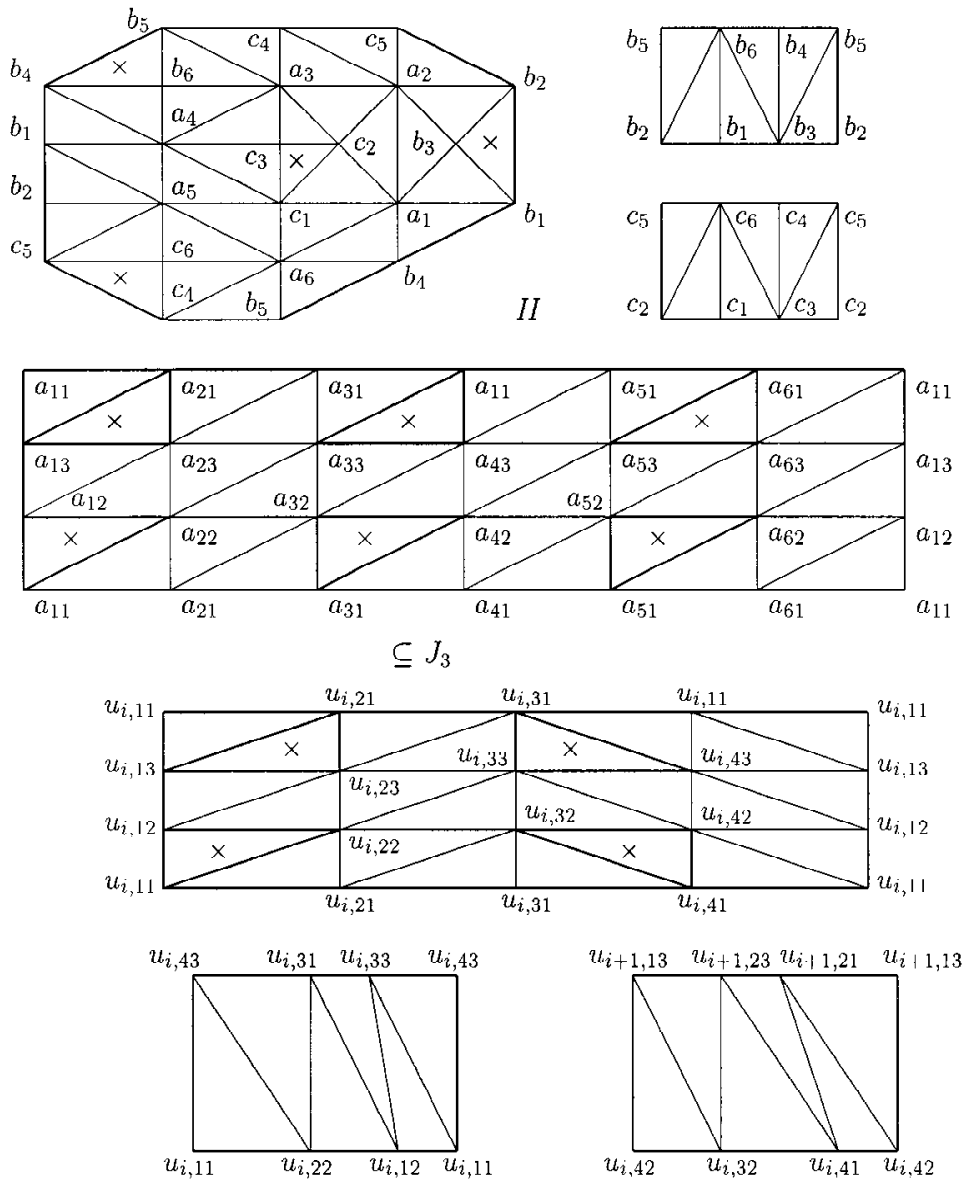

$u_{i \mid 1,13} \quad u_{i+1,23} u_{i \mid 1,21} \quad u_{i \mid 1,13}$
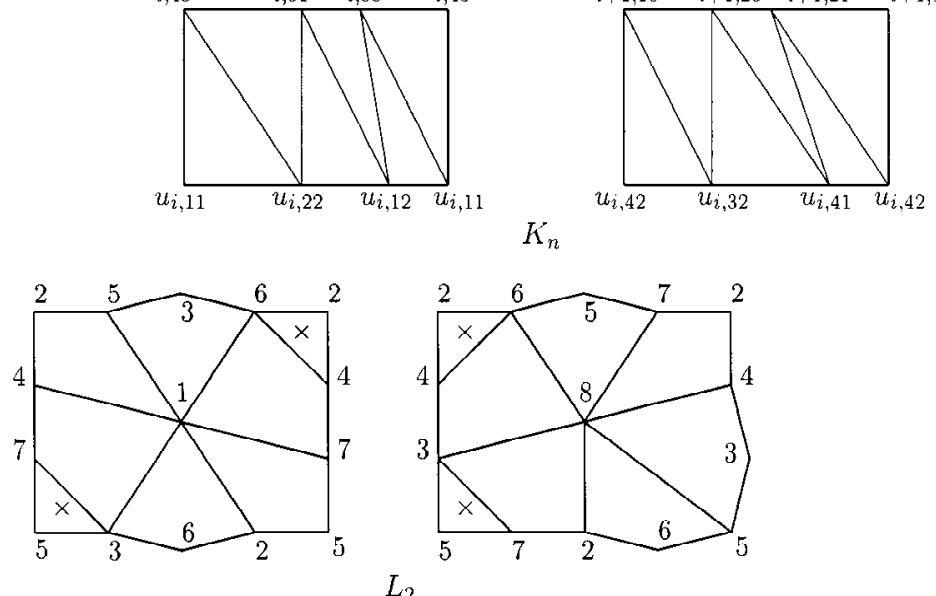


\begin{tabular}{|c|c|c|c|c|c|}
\hline $\begin{array}{l}a_{11} \\
b_{14}-a_{51}\end{array}$ & $a_{21}$ & $\begin{array}{l}a_{31} \\
b_{34}-a_{11}\end{array}$ & $a_{41}$ & $\begin{array}{l}a_{51} \\
a_{54}-a_{31}\end{array}$ & $a_{61}$ \\
\hline$x$ & $b_{24}=a_{52}$ & $x$ & $b_{14}=a_{12}$ & $x$ & $b_{61}=a_{32}$ \\
\hline $\begin{array}{l}b_{13}=a_{61} \\
a_{12}\end{array}$ & $b_{23}=a_{62}$ & $\begin{array}{l}b_{33}=a_{21} \\
a_{32}\end{array}$ & $b_{43}=a_{22}$ & $\begin{array}{l}b_{53}=a_{41} \\
a_{52}\end{array}$ & $b_{63}=a_{42}$ \\
\hline$\times$ & $a_{22}$ & $x$ & $a_{42}$ & $\times$ & $a_{62}$ \\
\hline$a_{11}$ & $a_{21}$ & $\begin{array}{l}a_{31} \\
\\
L_{3}\end{array}$ & $a_{41}$ & $a_{51}$ & $a_{61}$ \\
\hline
\end{tabular}

\begin{tabular}{|c|c|c|c|}
\hline $\begin{array}{l}a_{i, 11} \\
a_{i, 14}\end{array}$ & $a_{i, 21}$ & $\begin{array}{l}a_{i, 31} \\
a_{i, 34}\end{array}$ & $a_{i, 41}$ \\
\hline$x$ & $a_{\imath, 24}$ & $x$ & $a_{i, 44}$ \\
\hline $\begin{array}{l}a_{i, 13} \\
a_{2,12} \\
\end{array}$ & $a_{i, 23}$ & $\begin{array}{l}a_{i, 33} \\
a_{i, 32}\end{array}$ & $a_{i, 43}$ \\
\hline$x$ & $a_{i, 22}$ & $x$ & $a_{i, 42}$ \\
\hline
\end{tabular}

\begin{tabular}{|c|c|c|c|c|}
\hline $\begin{array}{l}a_{i+1,11} \\
a_{i+1,14}\end{array}$ & $a_{i+1,21}$ & $\begin{array}{l}a_{i+1,31} \\
a_{i+1,34}\end{array}$ & $a_{i+1,11}$ & $a_{i+1,11}$ \\
\hline$x$ & $a_{2+1,24}$ & $x$ & $a_{2+1,44}$ & $a_{i+1,14}$ \\
\hline $\begin{array}{l}a_{i+1,13} \\
a_{i+1,12} \\
\end{array}$ & $a_{i+1,23}$ & $\begin{array}{l}a_{i+1,33} \\
a_{i+1,32}\end{array}$ & $a_{i+1,43}$ & $a_{i+1,13}$ \\
\hline$x$ & $a_{i+1,22}$ & $x$ & $a_{i+1,42}$ & $a_{i+1,12}$ \\
\hline
\end{tabular}

$P_{n}$
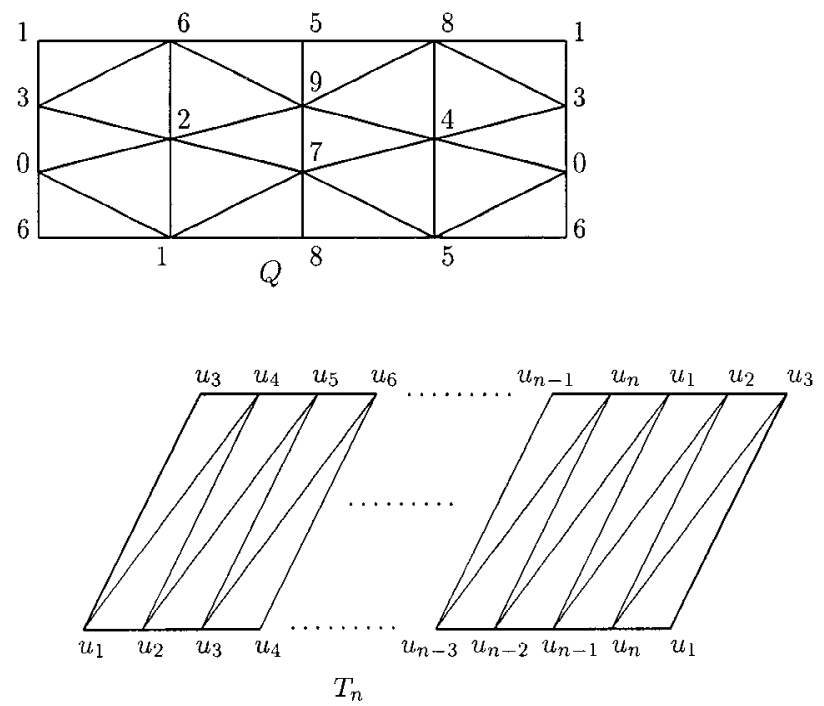


\section{Proofs}

Proof of Theorem 1. First observe that if $K$ is an equivelar polyhedron of type $\{p, q\}$, then $\widetilde{K}$ is an equivelar polyhedron of type $\{q, p\}$. Thus, $(p, q) \in \Phi(m)$ implies $(q, p) \in$ $\Phi(m)$.

If $K$ is a $\{p, q\}$-equivelar polyhedron with $f_{0}$ vertices, $f_{1}$ edges and $f_{2}$ faces, then

$$
q f_{0}=2 f_{1}=p f_{2} .
$$

This gives

$$
\frac{1}{p}+\frac{1}{q}-\frac{1}{2}=\frac{\chi(K)}{2 f_{1}}=\frac{\chi(K)}{q f_{0}} .
$$

Thus, $(p, q) \in \Phi(n)$, for $n \geq 0$, implies $1 / p+1 / q-\frac{1}{2} \geq 0$ and $(p, q) \in \Phi(-m)$, for $m>0$, implies $1 / p+1 / q-\frac{1}{2}<0$. This implies (i).

If there esists a $\{p, q\}$-equivelar polyhedron $K$ with $\chi(K)>0$, then $1 / p+1 / q>\frac{1}{2}$ or $(p-2)(q-2)<4$. This implies $(p, q) \in\{(3,3),(3,4),(4,3),(3,5),(5,3)\}$. Thus

$$
\Phi(1), \Phi(2) \subseteq\{(3,3),(3,4),(4,3),(3,5),(5,3)\} .
$$

From Example 1 we have $(3,3),(3,4),(4,3),(3,5),(5,3) \in \Phi(2)$. This proves (ii).

Let $K$ be an equivelar polyhedron of type $\{p, q\}$ and $\chi(K)=1$. If $(p, q)=(3,3)$, then, from (2), we get $f_{1}=3$, which is not possible. If $(p, q)=(3,4)$, then, from (2), $f_{0}=3$, which is again not possible. Similarly, $(p, q) \neq(4,3)$. Therefore, from (3), $(p, q)=(3,5)$ or $(5,3)$. This and Example $1\left(\mathbb{R} P_{6}^{2}\right.$ and $\left.R\right)$ imply (iii).

If there exists a $\{p, q\}$-equivelar polyhedron $K$ with $\chi(K)=0$, then, by (2), $1 / p+$ $1 / q=\frac{1}{2}$. Since $p, q \geq 3,(p, q)=(3,6),(6,3)$ or $(4,4)$. This together with Example 2 proves (iv).

Let $K$ be an equivelar polyhedron of type $\{p, q\}$ and $\chi(K)=-1$. Since, the existence of an equivelar polyhedron of type $\{p, q\}$ implies the existence of an equivelar polyhedron of type $\{q, p\}$, therefore we may assume $q \geq p$. Also $p, q \geq 3$ and $f_{0} \geq p+1$.

If $p=3$, then from (1) we get $-1=f_{0}-f_{1} / 3=f_{0}-q f_{0} / 6$ or $(q-6) f_{0}=6$. This implies $f_{0}=6$ and $q=7$, a contradiction. If $p=4$, then $-1=f_{0}-f_{1} / 2=f_{0}-q f_{0} / 4$ or $(q-4) f_{0}=4$, which is not possible. If $p \geq 5$, then $f_{0} \geq 6$ and hence $f_{1} \geq(6 \times 3) / 2=9$. Then, from (2), $1 / q=\frac{1}{2}-1 / p-1 /\left(2 f_{1}\right)>\frac{1}{2}-\frac{1}{5}-\frac{1}{18}>\frac{1}{5}$. This implies $q<5$, a contradiction to the assumption that $p \leq q$. This proves (v).

Observe that $F_{1}, H_{1}, \widetilde{F}_{1}, \widetilde{H}_{1} \in \Sigma(-2)$ and $F_{1}$ and $H_{1}$ are equivelar of type $\{4,5\}$ and $\{3,7\}$, respectively. Therefore, $\Phi(-2) \supseteq\{(5,4),(4,5),(7,3),(3,7)\}$.

Let $K$ be an equivelar polyhedron of type $\{p, q\}$ and $\chi(K)=-2$. In this case also we may assume that $3 \leq p \leq q \leq f_{0}-1$.

If $p \geq 5$, then $f_{0} \geq q+1 \geq 6$. Then, from (2), $\frac{1}{2}=1 / p+1 / q+2 /\left(q f_{0}\right) \leq$ $\frac{1}{5}+\frac{1}{5}+\frac{2}{5 \times 6}=\frac{14}{30}$, a contradiction.

If $p=4$, then $-2=f_{0}-f_{1} / 2=f_{0}-q f_{0} / 4$ or $(q-4) f_{0}=8$, which implies $\left(f_{0}, q\right)=(8,5)$.

If $p=3$, then from (1) we get $-2=f_{0}-f_{1} / 3=f_{0}-q f_{0} / 6$ or $(q-6) f_{0}=12$. This implies $\left(f_{0}, q\right)=(12,7)$. These imply $\Phi(-2) \subseteq\{(5,4),(4,5),(7,3),(3,7)\}$. This proves (vi). 
Observe that $H, M_{1}, G \in \Sigma(-3)$ and $H, M_{1}, G$ are equivelar of type $(3,7),(3,8)$ and $(4,5)$, respectively. Thus, $\{(3,7),(7,3),(3,8),(8,3),(4,5),(5,4)\} \subseteq \Phi(-3)$.

Let $K$ be an equivelar polyhedron of type $\{p, q\}$ and $\chi(K)=-3$. Assume $p \leq q \leq$ $f_{0}-1$.

If $p=3$, then, by (1), $(q-6) f_{0}=18$. This implies $\left(f_{0}, q\right)=(9,8)$ or $(18,7)$.

If $p=4$, then, by (1), $(q-4) f_{0}=12$, which implies $\left(f_{0}, q\right)=(12,5)$.

If $p=q=5$, then $f_{0}=6$. Now, $S_{1}=\{12345,15264,14536,16423,13652,24356\}$ is a $\{5,5\}$-equivelar polyhedron of Euler characteristic -3 . So, $(5,5) \in \Phi(-3)$.

Finally, assume that $p \geq 5$ and $q \geq 6$. Then, by (2), $\frac{1}{2} \leq \frac{1}{5}+\frac{1}{6}+\frac{3}{6 \times 7}=\frac{92}{210}$, a contradiction. This proves (vii).

$H_{m}$ (in Example 4) is an equivelar polyhedron of type $\{3,7\}$ and of Euler characteristic $-2 m$ for all $m \geq 1$, therefore $(3,7)$ (and hence $(7,3)) \in \Phi(-2 m)$ for all $m \geq 1$.

$K_{1}$ (in Example 5) is an equivelar polyhedron of type $\{3,8\}$ of Euler characteristic -4 and $J_{m}$ (in Example 5) is an equivelar polyhedron of type $\{3,8\}$ and of Euler characteristic $-2 m$ for all $m \geq 3$. Therefore $(3,8)$ (and hence $(8,3)) \in \Phi(-2 m)$ for all $m \geq 2$.

$F_{m}$ (in Example 3) is an equivelar polyhedron of type $\{4,5\}$ of Euler characteristic $-2 m$ for all $m \geq 2$. Therefore $(4,5)$ (and hence $(5,4)) \in \Phi(-2 m)$ for all $m \geq 2$.

$L_{m}$ (in Example 6) is an equivelar polyhedron of type $\{4,6\}$ of Euler characteristic $-2 m$ for all $m \geq 2$. Therefore $(4,6)$ (and hence $(6,4)) \in \Phi(-2 m)$ for all $m \geq 2$. Thus,

$$
\begin{gathered}
\{(3,7),(7,3),(3,8),(8,3),(4,5),(5,4),(4,6),(6,4)\} \\
\subseteq \Phi(-2 m), \quad \text { for all } m \geq 2 .
\end{gathered}
$$

Let $K$ be an $f_{0}$-vertex equivelar polyhedron of type $\{p, q\}$ and $\chi(K)=-4$. Assume $p \leq q$, i.e., $3 \leq p \leq q \leq f_{0}-1$.

If $p \geq 5$ and $q \geq 6$, then $f_{0} \geq q+1 \geq 7$ and hence $\frac{1}{2}=1 / p+1 / q+4 /\left(q f_{0}\right) \leq$ $\frac{1}{5}+\frac{1}{6}+\frac{4}{6 \times 7}=\frac{97}{210}$, a contradiction.

If $p=q=5$, then, by $(2),\left(f_{0}, f_{1}\right)=(8,20)$ and hence $f_{2}=8$. For each $m \geq 2$, $S_{2 m}=\left\{a_{i} a_{i+1} b_{i+m+1} b_{i+m} b_{i+m-1}, a_{i} a_{i+1} b_{i+1} a_{i+m+1} b_{i}: 1 \leq i \leq 2 m\right.$, additions in the subscripts are modulo $2 m\}$ is $\{5,5\}$-equivelar and belongs to $\Sigma(-2 m)$. So, $(5,5) \in$ $\Phi(-2 m)$ for all $m \geq 2$.

If $p=3$, then, from (1), $-4=f_{0}-f_{1} / 3=f_{0}-q f_{0} / 6$ or $(q-6) f_{0}=24$. This implies $\left(f_{0}, q\right)=(12,8)$ or $(24,7)$ and hence $(p, q)=(3,8)$ or $(3,7)$.

If $p=4$, then, from (1), $-4=f_{0}-q f_{0} / 4$ or $(q-4) f_{0}=16$. This implies $\left(f_{0}, q\right)=$ $(16,5)$ or $(8,6)$ and hence $(p, q)=(4,5)$ or $(4,6)$. Thus, $(p, q) \in \Phi(-4)$ and $p \leq q$ imply $(p, q)=(3,8),(3,7),(4,5),(5,5)$ or $(4,6)$. So, $\Phi(-4) \subseteq\{(3,7),(7,3),(3,8)$, $(8,3),(4,5),(5,4),(4,6),(6,4),(5,5)\}$. This, the examples $S_{2 m}$ 's and (4) imply (viii).

(ix) follows from the proposition stated in the previous section.

Let $K$ be an equivelar polyhedron of type $\{p, q\}$ and $\chi(K)=-m$, where $m>0$. In this case also, we may first assume that $p \leq q$, i.e., $3 \leq p \leq q \leq f_{0}-1$. Then, by (2), $\frac{1}{2}-1 / p=1 / q+m /\left(q f_{0}\right) \leq 1 / q+m /(q(q+1))$. This gives

$$
\begin{aligned}
2 p(q+1+m) & \geq q(q+1)(p-2) \geq p(q+1)(p-2) \quad \text { or } \\
2 m & \geq(q+1)(p-4) .
\end{aligned}
$$

Clearly, if $p>4$, then there are only finitely many $q$ such that $(p, q)$ satisfies (6). So, there are only finitely many $(p, q) \in \Phi(-m)$ such that $p, q>4$. 


$$
\begin{aligned}
& \text { If } p=3 \text {, then from (5) we get } 6(q+1+m) \geq 3 q(q+1) \text { or } \\
& \qquad 6 m \geq(q-6)(q+1) .
\end{aligned}
$$

Clearly, given $m>0$, only finitely many $q(\geq 3$ ) satisfy (7). This shows that there exists only finitely many $q$ such that $(3, q) \in \Phi(-m)$.

If $q \geq p=4$, then from (5) we get $4(q+1+m) \geq q(q+1)$ or

$$
4 m \geq(q-4)(q+1) .
$$

Clearly, given $m>0$, only finitely many $q(\geq 4)$ satisfy (8). This shows that there exists only finitely many $q \geq 4$ such that $(4, q) \in \Phi(-m)$. Therefore there are only finitely many $(p, q) \in \Phi(-m)$ such that $p$ or $q$ is 4 . So, $\Phi(-m)$ is finite for all $m>0$. This together with (ii), (iii) and (iv) imply (x).

For each $n \geq 7, T_{n}$ is an $n$-vertex $\{3,6\}$-equivelar polyhedron. This proves (xi).

Proof of Corollary 2. Let $(p, q) \in \Phi(n)$. Let $K$ be an equivelar polyhedron of type $\{p, q\}$ and $\chi(K)=n$. If $n \neq 0$, then, by (2) and (1), $\left(f_{0}(K), f_{1}(K), f_{2}(K)\right)$ is uniquely determined by $(p, q)$. Since, for each $f_{0}$, there exist finitely many polyhedra on $f_{0}$ vertices, therefore for a given $(p, q) \in \Phi(n)$ there are only finitely many equivelar polyhedra of type $\{p, q\}$. The corollary now follows from Theorem 1(x).

Lemma 1. If $K$ is a 9-vertex $\{3,6\}$-equivelar polyhedron, then $K$ is isomorphic to $T_{9}$, $A_{3,3}$ or $B_{3,3}$ defined in Example 2.

Proof. Let $K$ be a 9-vertex $\{3,6\}$-equivelar polyhedron. Then $f_{2}(K)=18$ and $\operatorname{NEG}(K)$ is a 2-regular graph and hence is either a cycle or disjoint union of cycles on nine vertices. So, $\operatorname{NEG}(K)$ is isomorphic to $C_{9}, C_{6} \sqcup C_{3}, C_{5} \sqcup C_{4}$ or $3 C_{3}:=C_{3} \sqcup C_{3} \sqcup C_{3}$. (Here $C_{n}$ denotes the cycle with $n$ vertices. A cycle with edges $v_{1} v_{2}, \ldots, v_{n-1} v_{n}, v_{n} v_{1}$ is also denoted by $C_{n}\left(v_{1}, \ldots, v_{n}\right)$.)

If $\operatorname{NEG}(K)=C_{5} \sqcup C_{4}$, then there exist four vertices, $a, b, c, d$ say, such that $a c$ and $b d$ are edges in $K$ but $a b, b c, c d$ and $d a$ are not edges in $K$. Consider the following six sets of faces, $S_{a c}=\{\sigma: a, c \in \sigma\}, S_{b d}=\{\sigma: b, d \in \sigma\}, S_{a}=\{\sigma: a \in \sigma, c \notin \sigma\}, S_{b}=\{\sigma: b \in$ $\sigma, d \notin \sigma\}, S_{c}=\{\sigma: c \in \sigma, a \notin \sigma\}$ and $S_{d}=\{\sigma: d \in \sigma, b \notin \sigma\}$. Clearly, these six sets are pairwise disjoint and $\#\left(S_{a c}\right)=\#\left(S_{b d}\right)=2$ and $\#\left(S_{a}\right)=\#\left(S_{b}\right)=\#\left(S_{c}\right)=\#\left(S_{d}\right)=4$. This implies that $f_{2}(K) \geq 20$, a contradiction. So, $\operatorname{NEG}(K)$ is $C_{9}, C_{6} \sqcup C_{3}$ or $3 C_{3}$.

First consider the case when the non-edge graph consists of three 3-cycles, i.e., $\operatorname{NEG}(K)=C_{3}(1,2,3) \sqcup C_{3}(4,5,6) \sqcup C_{3}(7,8,9) \cong \operatorname{NEG}\left(A_{3,3}\right)$. Then, up to an isomorphism, $\operatorname{Lk}_{K}(1)=C_{6}(4,7,5,9,6,8)$ and hence we may assume, without loss, $\mathrm{Lk}_{K}(4)=C_{6}(1,7,2,9,3,8)$. These imply $\operatorname{Lk}_{K}(7)=C_{6}(1,4,2,6,3,5), \mathrm{Lk}_{K}(8)=$ $C_{6}(1,6,2,5,3,4)$. These imply $\operatorname{Lk}_{K}(2)=C_{6}(4,9,5,8,6,7), \operatorname{Lk}_{K}(3)=C_{6}(4,8,5,7$, $6,9), \operatorname{Lk}_{K}(5)=C_{6}(1,9,2,8,3,7), \operatorname{Lk}_{K}(6)=C_{6}(1,8,2,7,3,9)$ and hence $\operatorname{Lk}_{K}(9)=$ $C_{6}(1,5,2,4,3,6)$. Clearly, these imply that $K$ is unique, up to an isomorphism, in this case and hence is isomorphic to $A_{3,3}$.

Now assume $\operatorname{NEG}(K)=C_{6}(1, \ldots, 6) \sqcup C_{3}(7,8,9) \cong \operatorname{NEG}\left(B_{3,3}\right)$. Then, we may assume, $\operatorname{Lk}_{K}(1)=C_{6}(3,8,4,9,5,7)$. This implies $\operatorname{Lk}_{K}(7)=C_{6}(1,5,2,4,6,3)$. Then $\mathrm{Lk}_{K}(3)=C_{6}(1,8,5,9,6,7)$ and $\mathrm{Lk}_{K}(5)=C_{6}(1,7,2,8,3,9)$. These imply $\mathrm{Lk}_{K}(8)=$ 
$C_{6}(1,3,5,2,6,4), \operatorname{Lk}_{K}(9)=C_{6}(1,4,2,6,3,5), \operatorname{Lk}_{K}(2)=C_{6}(4,7,5,8,6,9), \operatorname{Lk}_{K}(6)$ $=C_{6}(2,9,3,7,4,8)$. So, $\operatorname{Lk}_{K}(4)=C_{6}(1,9,2,7,6,8)$. These determine the polyhedron uniquely and therefore $K$ is isomorphic to $B_{3,3}$.

Finally assume $\operatorname{NEG}(K)=C_{9}(1, \ldots, 9)$. Then $\operatorname{Lk}_{K}(1)=C_{6}(4,6,8,3,5,7), C_{6}(4$, $6,8,5,3,7), C_{6}(4,6,3,8,5,7), C_{6}(4,7,5,3,6,8)$ or $C_{6}(4,6,3,7,5,8)$.

If $\operatorname{Lk}_{K}(1)=C_{6}(4,6,8,3,5,7)$, then $\operatorname{Lk}_{K}(3)=C_{6}(1,5,7,9,6,8)$. These imply $\operatorname{Lk}_{K}(5)=C_{3}(1,3,7)$, a contradiction. If $\operatorname{Lk}_{K}(1)=C_{6}(4,6,3,8,5,7)$, then $\operatorname{Lk}_{K}(3)=$ $C_{6}(1,6,9,7,5,8)$. These give $\mathrm{Lk}_{K}(5)=C_{4}(1,7,3,8)$, a contradiction. If $\mathrm{Lk}_{K}(1)=$ $C_{6}(4,7,5,3,6,8)$, then 15,16 are edges and $67,78,89$ are non-edges in $\operatorname{Lk}_{K}(3)$. It is then not possible to construct $\operatorname{Lk}_{K}(3)$, which is a 6-cycle with vertex-set $\{1,5,6,7,8,9\}$. Similarly, it is not possible to construct $\operatorname{Lk}_{K}(8)$ when $\operatorname{Lk}_{K}(1)=C_{6}(4,6,8,5,3,7)$. Thus, $\operatorname{Lk}_{K}(1)=C_{6}(4,6,3,7,5,8)$.

Similarly, replacing 1 by $i, \operatorname{Lk}_{K}(i)=C_{6}(i+3, i+5, i+2, i+6, i+4, i+$ 7 ), for $1 \leq i \leq 9$ (additions in the subscripts are modulo 9). This shows that the simplicial polyhedron is unique, up to an isomorphism, with the non-edge graph a 9cycle. Therefore, $K$ is isomorphic to $T_{9}$. This completes the proof.

Lemma 2. If $K$ is a 10-vertex $\{3,6\}$-equivelar polyhedron, then $K$ is isomorphic to $T_{10}$ or $Q$ defined in Example 2.

Proof. Let $K$ be a 10 -vertex $\{3,6\}$-equivelar polyhedron. Choose a vertex, say $u_{0}$. Let the link of $u_{0}$ be $C_{6}\left(u_{1}, \ldots, u_{6}\right)$. Since the link of each vertex is a 6-cycle, $u_{i} u_{i+1} u_{i+2}$ is not a face in $K$ for $i=1, \ldots, 6$ (additions in the subscripts are modulo 6). If either $u_{i} u_{i+1} u_{i+3}$ or $u_{i} u_{i+1} u_{i+4}$ is a face for each $i$, then we get 12 faces and hence the number of faces through the remaining three vertices is $\leq 8$, a contradiction. So, assume $u_{1} u_{2} u_{7}$ is a face, where $u_{7}$ is one of the remaining three vertices. Then $u_{1} u_{6} u_{7}$ and $u_{2} u_{3} u_{7}$ cannot be faces.

Case 1: $u_{1} u_{3} u_{6}$ or $u_{2} u_{3} u_{6}$ is a face. Assume, without loss, that $u_{1} u_{3} u_{6}$ is a face.

If $u_{5}$ is in the link of $u_{1}$, then $u_{1} u_{3} u_{5}, u_{1} u_{5} u_{7}$ are faces and hence $V\left(\operatorname{Lk}\left(u_{3}\right)\right)=$ $\left\{u_{0}, u_{1}, u_{2}, u_{4}, u_{5}, u_{6}\right\}$. This gives 12 faces through $u_{0}, u_{1}$ and $u_{3}$. Hence, the number of faces through the remaining two vertices is $\leq 8$, a contradiction. If $u_{4}$ is in the link of $u_{1}$, then $u_{1} u_{3} u_{4}$ and $u_{1} u_{4} u_{7}$ are faces. Here, five vertices of each of $\operatorname{Lk}\left(u_{3}\right)$ and $\operatorname{Lk}\left(u_{4}\right)$ are known. This implies $V\left(\operatorname{Lk}\left(u_{8}\right)\right)=\left\{u_{2}, u_{3}, u_{5}, u_{6}, u_{7}, u_{9}\right\}$ and $V\left(\operatorname{Lk}\left(u_{9}\right)\right)=$ $\left\{u_{2}, u_{4}, u_{5}, u_{6}, u_{7}, u_{8}\right\}$, where $u_{8}$ and $u_{9}$ are the remaining two vertices. Therefore, $\operatorname{Lk}\left(u_{4}\right)=C_{6}\left(u_{7}, u_{1}, u_{3}, u_{0}, u_{5}, u_{9}\right)$ and $\operatorname{Lk}\left(u_{3}\right)=C_{6}\left(u_{2}, u_{0}, u_{4}, u_{1}, u_{6}, u_{8}\right)$. Then $\operatorname{Lk}\left(u_{2}\right)=C_{6}\left(u_{7}, u_{1}, u_{0}, u_{3}, u_{8}, u_{9}\right)$. However, now $\operatorname{Lk}\left(u_{7}\right)$ contains $C_{4}\left(u_{1}, u_{4}, u_{9}, u_{2}\right)$, which is impossible. Therefore, the sixth vertex in the link of $u_{1}$ is one of the remaining two vertices, say $u_{8}$.

Clearly, $u_{1} u_{3} u_{8}$ and $u_{1} u_{7} u_{8}$ are faces. Thus, $V\left(\operatorname{Lk}\left(u_{3}\right)\right)=\left\{u_{0}, u_{1}, u_{2}, u_{4}, u_{6}, u_{8}\right\}$. Let $u_{9}$ be the remaining vertex. Then $V\left(\operatorname{Lk}\left(u_{9}\right)\right)=\left\{u_{2}, u_{4}, u_{5}, u_{6}, u_{7}, u_{8}\right\}$. The link of $u_{2}$ shows that $u_{2} u_{7} u_{9}$ is a face. Now, from the link of $u_{3}$, either $u_{2} u_{3} u_{8}$ and $u_{3} u_{4} u_{6}$ are faces or $u_{2} u_{3} u_{6}$ and $u_{3} u_{4} u_{8}$ are faces. In either case, $u_{2} u_{5}$ is not an edge and hence $V\left(\operatorname{Lk}\left(u_{5}\right)\right)=\left\{u_{0}, u_{4}, u_{6}, u_{7}, u_{8}, u_{9}\right\}$.

Subcase 1.1: $u_{2} u_{3} u_{6}$ and $u_{3} u_{4} u_{8}$ are faces. Then, by considering $\operatorname{Lk}\left(u_{2}\right), \operatorname{Lk}\left(u_{6}\right)$ and $\operatorname{Lk}\left(u_{5}\right)$ successively, $u_{2} u_{6} u_{9}, u_{5} u_{6} u_{9}$ are faces and $u_{4} u_{5} u_{9}$ is not a face. Finally, by 
completing $\operatorname{Lk}\left(u_{9}\right)$ and $\operatorname{Lk}\left(u_{5}\right)$ successively, the other faces are $u_{4} u_{7} u_{9}, u_{4} u_{8} u_{9}, u_{5} u_{8} u_{9}$, $u_{4} u_{5} u_{7}$ and $u_{5} u_{7} u_{8}$. In this case $K$ is isomorphic, via the map $f: u_{i} \mapsto i$, to $Q$.

Subcase 1.2: $u_{2} u_{3} u_{8}$ and $u_{3} u_{4} u_{6}$ are faces. Then, by considering $\operatorname{Lk}\left(u_{2}\right), \operatorname{Lk}\left(u_{8}\right), \operatorname{Lk}\left(u_{5}\right)$ and $\operatorname{Lk}\left(u_{4}\right)$ successively, the other faces are $u_{2} u_{8} u_{9}, u_{5} u_{7} u_{8}, u_{5} u_{8} u_{9}, u_{4} u_{5} u_{7}, u_{5} u_{6} u_{9}$, $u_{4} u_{6} u_{9}, u_{4} u_{7} u_{9}$. So, $K$ is isomorphic, via the composition of $f$ and $(1,2,3)(4,8)(5,7)(6$, 9), to $T_{10}$.

Case 2: $u_{1} u_{4} u_{6}$ or $u_{2} u_{3} u_{5}$ is a face. Assume, without loss, that $u_{1} u_{4} u_{6}$ is a face. Let the remaining two vertices be $u_{8}$ and $u_{9}$.

If either $u_{1} u_{8}$ or $u_{1} u_{9}$ is an edge, say $u_{1} u_{8}$ is an edge, then, by considering $\operatorname{Lk}\left(u_{1}\right)$ and $\operatorname{Lk}\left(u_{4}\right)$, successively, $u_{1} u_{4} u_{8}, u_{1} u_{7} u_{8}, u_{3} u_{4} u_{6}$ and $u_{4} u_{5} u_{8}$ are faces. Clearly, $V\left(\operatorname{Lk}\left(u_{9}\right)\right)=$ $\left\{u_{2}, u_{3}, u_{5}, u_{6}, u_{7}, u_{8}\right\}$. By considering $\operatorname{Lk}\left(u_{6}\right)$ and $\operatorname{Lk}\left(u_{5}\right), u_{3} u_{6} u_{9}$ and $u_{5} u_{6} u_{9}$ are faces and $u_{2} u_{3} u_{5}$ is not a face. Then, from $\operatorname{Lk}\left(u_{3}\right), u_{2} u_{3} u_{8}$ and $u_{3} u_{8} u_{9}$ are faces. This gives $\operatorname{deg}\left(u_{8}\right)>6$, a contradiction. So, the sixth vertex in the link of $u_{1}$ is either $u_{3}$ or $u_{5}$.

If neither $u_{8}$ nor $u_{9}$ is in the link of $u_{4}$, then from the links of $u_{0}, u_{1}$ and $u_{4}$ we get 12 faces. Therefore, the number of faces containing $u_{8}$ or $u_{9}$ is $\leq 8$, a contradiction. So, $\operatorname{Lk}\left(u_{4}\right)$ contains one of $u_{8}$ or $u_{9}$, say $u_{8}$. Then $V\left(\operatorname{Lk}\left(u_{9}\right)\right)=\left\{u_{2}, u_{3}, u_{5}, u_{6}, u_{7}, u_{8}\right\}$.

Subcase 2.1: $u_{3}$ is in the link of $u_{1}$. The links of $u_{1}$ and $u_{4}$ show that $u_{1} u_{3} u_{4}, u_{1} u_{3} u_{7}$, $u_{4} u_{5} u_{8}$ and $u_{4} u_{6} u_{8}$ are faces. It is easy to see, by considering the link of $u_{3}$, that $u_{2} u_{3} u_{9}$ and $u_{3} u_{7} u_{9}$ are faces. However, this implies $V\left(\operatorname{Lk}\left(u_{8}\right)\right)=\left\{u_{2}, u_{4}, u_{5}, u_{6}, u_{7}, u_{9}\right\}$. Then, by completing $\operatorname{Lk}\left(u_{2}\right), \operatorname{Lk}\left(u_{6}\right)$ and $\operatorname{Lk}\left(u_{5}\right)$ successively, the other faces are $u_{2} u_{7} u_{8}$, $u_{2} u_{8} u_{9}, u_{5} u_{6} u_{9}, u_{6} u_{8} u_{9}, u_{5} u_{7} u_{8}$ and $u_{5} u_{7} u_{9}$. In this case $K$ is isomorphic, via the composition of $f$ and $(1,9,5,3,8,4)(2,7,6)$, to $T_{10}$.

Subcase 2.2: $u_{5}$ is in the link of $u_{1}$. Clearly, $u_{1} u_{4} u_{5}$ and $u_{1} u_{5} u_{7}$ are faces. In this case, by considering $\operatorname{Lk}\left(u_{4}\right), \operatorname{Lk}\left(u_{5}\right), \operatorname{Lk}\left(u_{6}\right), \operatorname{Lk}\left(u_{7}\right)$ and $\operatorname{Lk}\left(u_{2}\right), u_{3} u_{4} u_{8}, u_{4} u_{6} u_{8}, u_{5} u_{6} u_{9}$, $u_{5} u_{7} u_{9}, u_{6} u_{8} u_{9}, u_{2} u_{7} u_{8}, u_{3} u_{7} u_{8}, u_{3} u_{7} u_{9}, u_{2} u_{3} u_{9}$ and $u_{2} u_{8} u_{9}$ are faces. Then $K$ is isomorphic, via the map $\left(u_{1}, u_{6}\right)\left(u_{2}, u_{5}\right)\left(u_{3}, u_{4}\right)\left(u_{7}, u_{9}\right)$, to $Q$.

Case 3: None of $u_{1} u_{3} u_{6}, u_{1} u_{4} u_{6}, u_{2} u_{3} u_{5}$ or $u_{2} u_{3} u_{6}$ is a face. In this case we can assume that $u_{1} u_{6} u_{8}$ is a face, where $u_{8}$ is one of the remaining two vertices. Let $u_{9}$ be the remaining vertex.

If $u_{3} \in \operatorname{Lk}\left(u_{1}\right)$, then, by considering the links of $u_{1}$ and $u_{3}, u_{1} u_{3} u_{7}, u_{1} u_{3} u_{8}, u_{2} u_{3} u_{8}$ and $u_{3} u_{4} u_{7}$ are faces in $K$. Clearly, $V\left(\operatorname{Lk}\left(u_{9}\right)\right)=\left\{u_{2}, u_{4}, u_{5}, u_{6}, u_{7}, u_{8}\right\}$. The links of $u_{2}$ and $u_{8}$ show that $\operatorname{deg}\left(u_{7}\right)>6$, a contradiction. Similarly, we get a contradiction if $u_{5} \in \operatorname{Lk}\left(u_{1}\right)$.

If $u_{4} \in \operatorname{Lk}\left(u_{1}\right)$, then $u_{1} u_{4} u_{7}$ and $u_{1} u_{4} u_{8}$ are faces in $K$. Here, $V\left(\operatorname{Lk}\left(u_{9}\right)\right)=$ $\left\{u_{2}, u_{3}, u_{5}, u_{6}, u_{7}, u_{8}\right\}$. To complete $\operatorname{Lk}\left(u_{4}\right)$, either $u_{3} u_{4} u_{7}$ or $u_{3} u_{4} u_{8}$ has to be a face. In both cases we see that $u_{6} u_{8} u_{9} \in K$. In the first case, the links of $u_{4}$ and $u_{5}$ show that $u_{4} u_{5} u_{8}$ and $u_{5} u_{8} u_{9}$ are faces, which imply that $\operatorname{deg}\left(u_{8}\right)<6$, a contradiction. In the second case, $\operatorname{Lk}\left(u_{4}\right), \operatorname{Lk}\left(u_{5}\right)$ and $\operatorname{Lk}\left(u_{7}\right)$ show that $u_{4} u_{5} u_{7}, u_{5} u_{7} u_{9}, u_{2} u_{7} u_{8}$ and $u_{7} u_{8} u_{9}$ are faces. This implies $\operatorname{deg}\left(u_{8}\right)>6$, a contradiction. Hence, the sixth vertex in $\operatorname{Lk}\left(u_{1}\right)$ is $u_{9}$.

Clearly, the edge $u_{2} u_{3}$ belongs to either $u_{2} u_{3} u_{8}$ or $u_{2} u_{3} u_{9}$.

If $u_{2} u_{3} u_{8}$ is a face, the sixth vertex, $y$, in $\operatorname{Lk}\left(u_{2}\right)$, is one of $u_{4}, u_{5}$ or $u_{6}$. If $y=u_{4}$ or $u_{5}$, the links of $u_{2}$ and $y$ show that $\operatorname{deg}\left(u_{8}\right)>6$, a contradiction. If $y=u_{6}$, by considering the links of $u_{2}$ and $u_{6}$, we see that $u_{2} u_{6} u_{7}, u_{2} u_{6} u_{8}$ and $u_{5} u_{6} u_{7}$ are faces. 
Clearly, $u_{3} u_{4} u_{9} \in K$. The sixth vertex in $\operatorname{Lk}\left(u_{7}\right)$ has to be $u_{3}$. However, this implies that $\operatorname{deg}\left(u_{3}\right)>6$, a contradiction. Hence, $u_{2} u_{3} u_{9} \in K$.

The sixth vertex, say $x$, in $\operatorname{Lk}\left(u_{2}\right)$, is one of $u_{4}, u_{5}$ or $u_{8}$.

If $x=u_{4}$ or $u_{5}$, by considering the links of $x$ and $u_{9}$, we observe that $\operatorname{deg}\left(u_{9}\right)>6$, a contradiction. Hence, $x=u_{8}$.

The links of $u_{2}, u_{8}, u_{4}, u_{7}$ and $u_{3}$ show that $u_{2} u_{7} u_{8}, u_{2} u_{8} u_{9}, u_{4} u_{6} u_{8}, u_{4} u_{7} u_{8}, u_{3} u_{4} u_{6}$, $u_{4} u_{5} u_{7}, u_{5} u_{7} u_{9}, u_{3} u_{5} u_{6}$ and $u_{3} u_{5} u_{9}$ are faces. Here, $K$ is isomorphic, via the map $(1,4)(2,5)(3,6)$, to $Q$. This completes the proof.

Lemma 3. If $K$ is an 11-vertex $\{3,6\}$-equivelar polyhedron, then $K$ is isomorphic to $T_{11}$ defined in Example 2.

Proof. Let $K$ be an 11-vertex $\{3,6\}$-equivelar polyhedron. Choose a vertex, say $u_{0}$, and let the link of $u_{0}$ be $C_{6}\left(u_{1}, \ldots, u_{6}\right)$. By an argument similar to that in the previous lemma, we assume that $u_{1} u_{2} u_{7}$ is a face.

Claim. One of $u_{1} u_{3} u_{6}, u_{1} u_{4} u_{6}, u_{2} u_{3} u_{5}$ or $u_{2} u_{3} u_{6}$ has to be a face.

If not assume $u_{1} u_{6} u_{8}$ is a face, where $u_{8}$ is one of the remaining three vertices. Let $u_{9}$ and $u_{10}$ be the remaining two vertices.

If $u_{3} \in \operatorname{Lk}\left(u_{1}\right)$, then the links of $u_{1}$ and $u_{3}$ show that $u_{1} u_{3} u_{7}, u_{1} u_{3} u_{8}, u_{2} u_{3} u_{8}$ and $u_{3} u_{4} u_{7}$ are faces in $K$. It is clear that $\operatorname{Lk}\left(u_{2}\right)$ contains either $u_{9}$ or $u_{10}$, say $u_{9}$. Then $V\left(\operatorname{Lk}\left(u_{10}\right)\right)=\left\{u_{4}, u_{5}, u_{6}, u_{7}, u_{8}, u_{9}\right\}$. The links of $u_{8}$ and $u_{7}$ now show that $\operatorname{deg}\left(u_{9}\right)<6$, a contradiction.

If $u_{4} \in \operatorname{Lk}\left(u_{1}\right)$, then it is clear that the edge $u_{5} u_{6}$ belongs to $u_{5} u_{6} u_{7}$ or $u_{5} u_{6} u_{9}$ (or $\left.u_{5} u_{6} u_{10}\right)$. If $u_{5} u_{6} u_{7} \in K$, then $\operatorname{deg}\left(u_{9}\right), \operatorname{deg}\left(u_{10}\right)<6$. If $u_{5} u_{6} u_{9}\left(\right.$ or $\left.u_{5} u_{6} u_{10}\right)$ is a face, then, by considering $\operatorname{Lk}\left(u_{4}\right)$, either $u_{3} u_{4} u_{7}$ or $u_{3} u_{4} u_{8} \in K$. In both cases we see that the link of $u_{5}$ has only five vertices, a contradiction.

Finally, assume (without loss) that $u_{9} \in \operatorname{Lk}\left(u_{1}\right)$. The edge $u_{2} u_{3}$ can belong to one of $u_{2} u_{3} u_{8}, u_{2} u_{3} u_{9}$ or $u_{2} u_{3} u_{10}$.

If $u_{2} u_{3} u_{8} \in K$, then the sixth vertex, say $y$, in $\operatorname{Lk}\left(u_{2}\right)$ is $u_{4}, u_{5}, u_{6}$ or $u_{10}$. If $y=u_{4}$ or $u_{5}$, then $V\left(\operatorname{Lk}\left(u_{10}\right)\right)=\left\{u_{3}, u_{4}, u_{5}, u_{6}, u_{7}, u_{9}\right\} \backslash\{y\}$ and hence $\operatorname{deg}\left(u_{10}\right)<6$. If $y=u_{6}$, we can easily see that $V\left(\operatorname{Lk}\left(u_{10}\right)\right)=\left\{u_{3}, u_{4}, u_{5}, u_{7}, u_{8}, u_{9}\right\}$. Then, by completing the links of $u_{6}, u_{7}$ and $u_{8}$, we see that $\operatorname{deg}\left(u_{9}\right)=4$, a contradiction. If $y=u_{10}$, then we see that either $u_{3} u_{6} u_{8}$ or $u_{6} u_{8} u_{10}$ has to be a face to complete $\operatorname{Lk}\left(u_{8}\right)$. In both the cases we see that there exists no vertex $x\left(\neq u_{0}\right)$ such that $u_{3} u_{4} x$ is a face in $K$, a contradiction.

If $u_{2} u_{3} u_{9}$ is a face in $K$, then, by an argument similar to the one above, we see that $u_{4}, u_{5} \notin \operatorname{Lk}\left(u_{2}\right)$. Hence either $u_{8}$ or $u_{10} \in \operatorname{Lk}\left(u_{2}\right)$. If $u_{8} \in \operatorname{Lk}\left(u_{2}\right)$, it is easy to see that $u_{10} \in \operatorname{Lk}\left(u_{8}\right)$. The last vertex in $\operatorname{Lk}\left(u_{9}\right)$ has to be $u_{5}$. Completing the link of $u_{7}$, we observe that $\operatorname{deg}\left(u_{5}\right)>6$, a contradiction. If $u_{10} \in \operatorname{Lk}\left(u_{2}\right)$, then $u_{2} u_{7} u_{10}$ and $u_{2} u_{9} u_{10}$ are simplices. To complete $\operatorname{Lk}\left(u_{9}\right), u_{3} u_{7} u_{9}$ and $u_{8} u_{9} u_{10}$ have to be faces. It is clear that $u_{4} u_{6} u_{8} \in K$. The last vertex in $\operatorname{Lk}\left(u_{7}\right)$ is one of $u_{4}, u_{5}$ or $u_{6}$, all of which are impossible.

If $u_{2} u_{3} u_{10} \in K$, then the sixth vertex, $z, \operatorname{in} \operatorname{Lk}\left(u_{2}\right)$ is $u_{4}, u_{5}$ or $u_{8}$. If $z=u_{4}$, the links of $u_{2}$ and $u_{4}$ show that $u_{2} u_{4} u_{7}, u_{2} u_{4} u_{10}, u_{4} u_{5} u_{10}$ and $u_{3} u_{4} u_{7}$ have to be faces. It is easy to see that $\operatorname{Lk}\left(u_{3}\right)$ has only five vertices, a contradiction. If $z=u_{8}$, considering the links of $u_{2}, u_{8}, u_{9}, u_{7}, u_{3}$ and $u_{6}$, we see that $u_{4} u_{6} u_{7}$ and $u_{4} u_{5} u_{6}$ are faces, a contradiction. If 
$z=u_{5}$, to complete $\operatorname{Lk}\left(u_{5}\right)$, we see that either $u_{4} u_{5} u_{7}$ or $u_{4} u_{5} u_{10}$ is a face. In the first case the links of $u_{7}$ and $u_{4}$ show that $\operatorname{deg}\left(u_{10}\right)>6$. In the second case we see that there exists no vertex $x\left(\neq u_{1}\right)$ such that $u_{7} u_{9} x$ is a face. This proves the claim.

By the claim we can assume without loss that $u_{1} u_{3} u_{6}$ or $u_{1} u_{4} u_{6}$ is a face.

Case 1: $u_{1} u_{3} u_{6}$ is a face in $K$. Let $u$ be the sixth vertex in $\operatorname{Lk}\left(u_{1}\right)$.

If $u=u_{5}$, we see from the links of $u_{1}$ and $u_{3}$ that $\operatorname{deg}\left(u_{5}\right)>6$, a contradiction. If $u=u_{4}$, the links of $u_{1}$ and $u_{3}$ show that $u_{1} u_{3} u_{4}, u_{1} u_{4} u_{7}, u_{2} u_{3} u_{8}$ and $u_{3} u_{6} u_{8}$ are faces, where $u_{8}$ is one of the remaining three vertices. Let $u_{9}$ and $u_{10}$ be the other two vertices. Now, one of $u_{9}$ or $u_{10}$, say $u_{9}$, has to be in the link of $u_{2}$. Hence, $V\left(\operatorname{Lk}\left(u_{10}\right)\right)=$ $\left\{u_{4}, u_{5}, u_{6}, u_{7}, u_{8}, u_{9}\right\}$. Then it is easy to see that $V\left(\operatorname{Lk}\left(u_{9}\right)\right)=\left\{u_{2}, u_{5}, u_{7}, u_{8}, u_{10}\right\}$, a contradiction. So, $u$ is one of the remaining three vertices, say $u_{8}$.

Clearly, $u_{1} u_{3} u_{8}$ and $u_{1} u_{7} u_{8}$ are faces. Let $u_{9}$ and $u_{10}$ be the other two vertices. To complete $\operatorname{Lk}\left(u_{3}\right)$ either $u_{2} u_{3} u_{6}$ or $u_{2} u_{3} u_{8} \in K$. If $u_{2} u_{3} u_{6} \in K$, we see that the sixth vertex in both $\operatorname{Lk}\left(u_{2}\right)$ and $\operatorname{Lk}\left(u_{6}\right)$ is the same, say $u_{9}$. Then $\operatorname{deg}\left(u_{10}\right)<6$, a contradiction. So, $u_{2} u_{3} u_{8}$ and $u_{3} u_{4} u_{6} \in K$.

Clearly, $V\left(\operatorname{Lk}\left(u_{9}\right)\right)=\left\{u_{2}, u_{4}, u_{5}, u_{7}, u_{8}, u_{10}\right\}$ and $V\left(\operatorname{Lk}\left(u_{10}\right)\right)=\left\{u_{4}, u_{5}, u_{6}, u_{7}, u_{8}\right.$, $\left.u_{9}\right\}$. Then, by considering the links of $u_{2}, u_{6}, u_{4}, u_{8}$ and $u_{5}, u_{2} u_{7} u_{9}, u_{2} u_{8} u_{9}, u_{4} u_{6} u_{10}$, $u_{5} u_{6} u_{10}, u_{4} u_{5} u_{9}, u_{4} u_{9} u_{10}, u_{7} u_{8} u_{10}, u_{8} u_{9} u_{10}, u_{5} u_{7} u_{9}$ and $u_{5} u_{7} u_{10}$ are faces in $K$. Here, $K$ is isomorphic, via the map $(1,2,3)(4,9,6,10,7,5,8)$, to $T_{11}$.

Case 2: $u_{1} u_{4} u_{6}$ is a face in $K$. Let $u$ be the sixth vertex in $\operatorname{Lk}\left(u_{1}\right)$.

If $u=u_{5}$, then $V\left(\operatorname{Lk}\left(u_{4}\right)\right)=\left\{u_{0}, u_{1}, u_{3}, u_{5}, u_{6}, u_{8}\right\}$ and $V\left(\operatorname{Lk}\left(u_{6}\right)\right)=\left\{u_{0}, u_{1}, u_{4}\right.$, $\left.u_{5}, u_{8}, u_{9}\right\}$ (otherwise $f_{2}<22$ ), where $u_{8}$ and $u_{9}$ are two of the remaining three vertices. Then the vertex set of the link of the remaining vertex is a subset of $\left\{u_{2}, u_{3}, u_{7}, u_{8}, u_{9}\right\}$, a contradiction. If $u$ is one of the remaining three vertices, say $u_{8}$, then the links of $u_{1}$ and $u_{4}$ show that $u_{1} u_{4} u_{8}, u_{1} u_{7} u_{8}, u_{3} u_{4} u_{6}$ and $u_{4} u_{5} u_{8}$ are faces in $K$. The face other that $u_{0} u_{5} u_{6}$ having $u_{5} u_{6}$ as an edge has to be $u_{5} u_{6} u_{9}$ (in all other cases $f_{2}<22$ ), where $u_{9}$ is one of the remaining two vertices. If $u_{10}$ is the remaining vertex, then $V\left(\operatorname{Lk}\left(u_{10}\right)\right)=\left\{u_{2}, u_{3}, u_{5}, u_{7}, u_{8}, u_{9}\right\}$. The links of $u_{3}, u_{5}$ and $u_{10}$ imply that $\operatorname{deg}\left(u_{2}\right)=$ 5 , a contradiction. So, $u=u_{3}$.

Clearly, $u_{1} u_{3} u_{4}$ and $u_{1} u_{3} u_{7}$ are faces. The sixth vertex in $\operatorname{Lk}\left(u_{4}\right)$ has to be one of the three remaining vertices, say $u_{8}$. Then $u_{4} u_{5} u_{8}$ and $u_{4} u_{6} u_{8} \in K$. If $u_{9}$ and $u_{10}$ are the remaining two vertices, then $V\left(\operatorname{Lk}\left(u_{9}\right)\right)=\left\{u_{2}, u_{3}, u_{5}, u_{7}, u_{8}, u_{10}\right\}$ and $V\left(\operatorname{Lk}\left(u_{10}\right)\right)=$ $\left\{u_{2}, u_{5}, u_{6}, u_{7}, u_{8}, u_{9}\right\}$. The links of $u_{3}, u_{2}, u_{6}, u_{5}$ and $u_{7}$ show that $u_{2} u_{3} u_{9}, u_{3} u_{7} u_{9}$, $u_{2} u_{7} u_{10}, u_{2} u_{9} u_{10}, u_{5} u_{6} u_{10}, u_{6} u_{8} u_{10}, u_{5} u_{8} u_{9}, u_{5} u_{9} u_{10}, u_{7} u_{8} u_{9}$ and $u_{7} u_{8} u_{10}$ are faces in $K$. In this case, $K$ is isomorphic, via the map $(0,1)(2,9,7,8,5,4)(3,10,6)$, to $T_{11}$. This completes the proof of the lemma.

Proof of Theorem 4. Let $K$ be a $\{3, q\}$-equivelar simplicial polyhedron on $n(\leq 11)$ vertices.

Since $\chi(K)=0$ we have $q=6$ and hence $n \geq 7$.

It is not difficult to show (also see in [9]) that $T_{7}$ is the only (up to isomorphism) 7-vertex neighbourly combinatorial 2-manifold. Hence, if $n=7$, then $K$ is isomorphic to $T_{7}$.

The case $n=8$ follows from the classification of combinatorial 2-manifolds on eight vertices in [10]. (It is also not difficult, by a similar argument as in the proof of Lemma 1, 
to show that there exists a unique combinatorial 2-manifold with non-edge graph $4 K_{2}$ (the disjoint union of four edges). This implies that $K$ is isomorphic to $T_{8}$.)

If $n=9$, then, by Lemma $1, K$ is isomorphic to $A_{3,3}, B_{3,3}$ or $T_{9}$.

If $n=10$, then, by Lemma $2, K$ is isomorphic to or $T_{10}$ or $Q$.

If $n=11$, then, by Lemma 3, $K$ is isomorphic to $T_{11}$. This completes the proof.

Lemma 4. If $M_{1}$ and $M_{2}$ are as in Examples 1 and 7 , then $M_{1} ¥ M_{2}$.

Proof. For $1 \leq i \leq 2$, let $A\left(\Lambda\left(M_{i}\right)\right)$ denote the adjacency matrix of the graph $\Lambda\left(M_{i}\right)$. Let $\mathcal{P}_{i}(x)$ denote the characteristic polynomial of $A\left(\Lambda\left(M_{i}\right)\right)$. Then

$$
\begin{aligned}
\mathcal{P}_{1}(x)= & (x-3)(x-2)^{6}(x-1)^{3} x^{4}(x+1)^{3}(x+2)^{6}(x+3), \\
\mathcal{P}_{2}(x)= & (x-3)(x-2)^{4}(x-1) x^{4}(x+1)^{2}(x+2)^{2}\left(x^{2}-3\right)\left(x^{2}+2 x-1\right) \\
& \cdot\left(x^{3}+2 x^{2}-4 x-6\right)^{2}
\end{aligned}
$$

If $M_{1}$ and $M_{2}$ are isomorphic, then $\Lambda\left(M_{1}\right)$ and $\Lambda\left(M_{2}\right)$ are isomorphic as graphs and hence $\mathcal{P}_{1}(x)=\mathcal{P}_{2}(x)$. Clearly, $\mathcal{P}_{1}(x) \neq \mathcal{P}_{2}(x)$. Hence, $M_{1} \neq M_{2}$.

Lemma 5. If $N_{1}, \ldots, N_{14}$ are as in Examples 1 and 8 , then $N_{i} ¥ N_{j}$ for $1 \leq i \neq j \leq$ 14.

Proof. For $1 \leq i \leq 14$, let $A\left(\Lambda\left(N_{i}\right)\right)$ denote the adjacency matrix of the graph $\Lambda\left(N_{i}\right)$. Let $\mathcal{P}_{i}(x)$ denote the characteristic polynomial of $A\left(\Lambda\left(N_{i}\right)\right)$. Then

$$
\begin{aligned}
\mathcal{P}_{1}(x)= & (x-3)(x-2)(x-1)(x+1)^{2}(x+2)\left(x^{3}+x^{2}-5 x-3\right)^{2} \\
& \cdot\left(x^{9}-12 x^{7}+2 x^{6}+45 x^{5}-12 x^{4}-52 x^{3}+9 x^{2}+15 x+1\right)^{2}, \\
\mathcal{P}_{2}(x)= & (x-3)(x-1)^{9}(x+2)^{4}\left(x^{2}+3 x+1\right)^{3}\left(x^{2}-x-3\right)^{5} \\
\mathcal{P}_{3}(x)= & (x-3)(x-1)^{3}(x+2)\left(x^{2}-x-3\right)^{2}\left(x^{2}+x-1\right)^{3} \\
& \cdot\left(x^{5}+x^{4}-8 x^{3}-5 x^{2}+13 x+6\right)^{3} \\
\mathcal{P}_{4}(x)= & (x-3)(x-1)^{2} x^{2}\left(x^{13}-20 x^{11}+154 x^{9}+8 x^{8}-576 x^{7}-82 x^{6}+1073 x^{5}\right. \\
& \left.+272 x^{4}-893 x^{3}-316 x^{2}+235 x+96\right) \\
& \cdot\left(x^{12}+5 x^{11}-7 x^{10}-67 x^{9}-25 x^{8}+315 x^{7}+315 x^{6}-599 x^{5}-850 x^{4}\right. \\
& \left.\quad+358 x^{3}+781 x^{2}+57 x-144\right) \\
\mathcal{P}_{5}(x)= & (x-3)(x-1) x^{3}\left(x^{12}-20 x^{10}+154 x^{8}+4 x^{7}-570 x^{6}-38 x^{5}+1015 x^{4}\right. \\
& \left.\quad+110 x^{3}-723 x^{2}-90 x+93\right) \\
& \cdot\left(x^{13}+4 x^{12}-12 x^{11}-60 x^{10}+42 x^{9}+340 x^{8}\right. \\
& \left.\quad+2 x^{7}-910 x^{6}-269 x^{5}+1170 x^{4}+453 x^{3}-650 x^{2}-207 x+112\right), \\
\mathcal{P}_{6}(x)= & (x-3)(x-1) x\left(x^{2}-5\right)\left(x^{2}+3 x+1\right)\left(x^{2}-x-1\right) \\
& \cdot\left(x^{11}+2 x^{10}-14 x^{9}-25 x^{8}+72 x^{7}+111 x^{6}-162 x^{5}-210 x^{4}+145 x^{3}\right. \\
& \left.\quad+156 x^{2}-28 x-24\right) \\
& \cdot\left(x^{10}-14 x^{8}-x^{7}+68 x^{6}+11 x^{5}-132 x^{4}-30 x^{3}+81 x^{2}+12 x-8\right),
\end{aligned}
$$




$$
\begin{aligned}
\mathcal{P}_{7}(x)= & (x-3)(x-1)(x+1) \\
& \cdot\left(x^{27}+3 x^{26}-35 x^{25}-105 x^{24}+541 x^{23}+1621 x^{22}-4851 x^{21}\right. \\
& -14513 x^{20}+27857 x^{19}+83335 x^{18}-106646 x^{17}-320442 x^{16} \\
& +274411 x^{15}+836409 x^{14}-466133 x^{13}-1470839 x^{12}+497081 x^{11} \\
& +1695261 x^{10}-297277 x^{9}-1217051 x^{8}+70531 x^{7}+501773 x^{6} \\
& \left.+9836 x^{5}-105408 x^{4}-6268 x^{3}+9416 x^{2}+568 x-228\right)
\end{aligned}
$$

Now, $N_{i} \cong N_{j}$ implies $\Lambda\left(N_{i}\right)$ and $\Lambda\left(N_{j}\right)$ are isomorphic as graphs and hence $\mathcal{P}_{i}(x)=$ 
$\mathcal{P}_{j}(x)$. Since $\mathcal{P}_{i}(x)$ and $\mathcal{P}_{j}(x)$ have different prime factorizations $\mathcal{P}_{i}(x) \neq \mathcal{P}_{j}(x)$, for $1 \leq i \neq j \leq 14$. Hence, for $1 \leq i \neq j \leq 14, N_{i} ¥ N_{j}$.

Proof of Theorem 5. Let $K$ be a 9-vertex neighbourly simplicial equivelar polyhedron. Choose a vertex, say $u_{9}$, and let the link of $u_{9}$ be $C_{8}\left(u_{1}, \ldots, u_{8}\right)$.

Claim. There exists a face of the form $u_{i} u_{i+1} u_{i+3}$ or $u_{i} u_{i+1} u_{i+6}$ for some $i \in\{1$, $\ldots, 8\}$ (additions in the subscripts are modulo 8).

If possible, assume that either $u_{i} u_{i+1} u_{i+4}$ or $u_{i} u_{i+1} u_{i+5}$ is a face for each $i$. Then we can assume without loss that $u_{1} u_{2} u_{5}$ is a face in $K$. Then, by repeated use of the assumption, it is clear that $u_{1} u_{4} u_{8}, u_{4} u_{5} u_{8}, u_{3} u_{7} u_{8}$ and $u_{3} u_{4} u_{7}$ are faces in $K$. To complete $\operatorname{Lk}\left(u_{4}\right)$ and $\operatorname{Lk}\left(u_{7}\right), u_{2} u_{4} u_{6}, u_{2} u_{4} u_{7}, u_{1} u_{4} u_{6}$ and $u_{1} u_{6} u_{7}$ have to be faces, a contradiction. This proves the claim.

By the claim, we can assume without loss that $u_{1} u_{2} u_{4} \in K$. We observe that the edge $u_{1} u_{8}$ belongs to one of $u_{1} u_{3} u_{8}, u_{1} u_{5} u_{8}$ or $u_{1} u_{6} u_{8}$.

Case 1: $u_{1} u_{3} u_{8}$ is a face. By considering $\operatorname{Lk}\left(u_{1}\right)$, one of $u_{1} u_{3} u_{5}, u_{1} u_{3} u_{6}$ or $u_{1} u_{3} u_{7}$ is a face.

If $u_{1} u_{3} u_{7} \in K$, then, by considering the links of $u_{1}$ and $u_{5}, u_{1} u_{5} u_{6}, u_{1} u_{4} u_{6}$ and $u_{1} u_{5} u_{7}$ are faces and hence, by considering $\operatorname{Lk}\left(u_{7}\right), u_{3} u_{5} u_{7} \notin K$. Then, by considering the links of $u_{5}, u_{8}$ and $u_{6}, u_{2} u_{3} u_{5}, u_{3} u_{5} u_{8} \in K, u_{5} u_{7} u_{8} \notin K$ and hence $u_{2} u_{5} u_{7}, u_{4} u_{5} u_{8}$, $u_{2} u_{7} u_{8}, u_{2} u_{6} u_{8}, u_{4} u_{6} u_{8}, u_{2} u_{3} u_{6}$ and $u_{3} u_{6} u_{7}$ are faces. Then $u_{2} u_{3} u_{5}, u_{2} u_{3} u_{6}$ and $u_{2} u_{3} u_{9}$ are the faces through $u_{2} u_{3}$, a contradiction.

If $u_{1} u_{3} u_{5} \in K$, then, by considering $\operatorname{Lk}\left(u_{1}\right)$ and $\operatorname{Lk}\left(u_{6}\right), u_{1} u_{6} u_{7}, u_{1} u_{4} u_{6}, u_{1} u_{5} u_{7}$ are faces. The edge $u_{4} u_{6}$, belongs to either $u_{3} u_{4} u_{6}$ or $u_{4} u_{6} u_{8}$. In the first case, $\operatorname{Lk}\left(u_{3}\right)$ and $\operatorname{Lk}\left(u_{6}\right)$ show that $u_{2} u_{3} u_{6} \notin K$ and $u_{2} u_{5} u_{6}, u_{2} u_{6} u_{8}, u_{3} u_{6} u_{8}, u_{2} u_{3} u_{7}, u_{3} u_{5} u_{7} \in K$. However, this implies that $\operatorname{Lk}\left(u_{5}\right)$ contains $C_{3}\left(u_{3}, u_{1}, u_{7}\right)$, a contradiction. In the second case $u_{2} u_{3} \in \operatorname{Lk}\left(u_{6}\right)$. Considering $\operatorname{Lk}\left(u_{7}\right)$ and $\operatorname{Lk}\left(u_{5}\right), u_{2} u_{4} u_{7}, u_{3} u_{4} u_{7}, u_{2} u_{5} u_{7}$ and $u_{3} u_{7} u_{8} \in K$. Then $\operatorname{Lk}\left(u_{8}\right)$ contains $C_{4}\left(u_{7}, u_{9}, u_{1}, u_{3}\right)$, a contradiction. So, $u_{1} u_{3} u_{6} \in K$.

From $\operatorname{Lk}\left(u_{1}\right), u_{1} u_{5} u_{7}$ is a face and either $u_{1} u_{4} u_{5}$ or $u_{1} u_{4} u_{7}$ is a face.

If $u_{1} u_{4} u_{5} \in K$, then so is $u_{1} u_{6} u_{7}$. To complete $\operatorname{Lk}\left(u_{7}\right)$, either $u_{2} u_{4} u_{7}$ and $u_{3} u_{4} u_{7}$ or $u_{2} u_{4} u_{7}$ and $u_{2} u_{3} u_{7}$ have to be faces. This shows that $\operatorname{deg}\left(u_{4}\right)<8$ in the first case and $\operatorname{deg}\left(u_{2}\right)<8$ in the second case, a contradiction. Thus, $u_{1} u_{4} u_{7} \in K$.

Clearly, $u_{1} u_{5} u_{6}$ is a face. By considering the links of $u_{3}$ and $u_{6}, u_{3} u_{6} u_{8} \notin K$ and $u_{4} u_{6} u_{8}, u_{2} u_{6} u_{8} \in K$. Then, by considering the links of $u_{6}, u_{4}$ and $u_{8}, u_{2} u_{4} u_{6}, u_{2} u_{4} u_{7}$ and $u_{2} u_{4} u_{8}$ are not faces and hence $u_{2} u_{4} u_{5}$ is a face. Now, by considering the links of $u_{2}, u_{5}$ and $u_{4}, u_{2} u_{5} u_{7}$ is not a face and $u_{2} u_{5} u_{8}, u_{2} u_{6} u_{7}, u_{2} u_{3} u_{7}, u_{3} u_{5} u_{7}, u_{3} u_{5} u_{8} u_{3} u_{4} u_{6}$, $u_{4} u_{7} u_{8}$ are faces. Here, $K$ is $M_{2}$.

Case 2: $u_{1} u_{5} u_{8}$ is a face. Clearly, one of $u_{1} u_{3} u_{5}, u_{1} u_{5} u_{6}$ or $u_{1} u_{5} u_{7}$ is a face.

Subcase 2.1: $u_{1} u_{3} u_{5}$ is a face. If $u_{1} u_{3} u_{7}$ is a face, then the link of $u_{1}$ shows that $u_{1} u_{4} u_{6}$ and $u_{1} u_{6} u_{7}$ are faces. By considering the links of $u_{3}, u_{8}, u_{7}, u_{4}$ and $u_{2}, u_{2} u_{5} u_{7}, u_{4} u_{5} u_{7}$, $u_{2} u_{3} u_{7}, u_{4} u_{7} u_{8}, u_{3} u_{4} u_{6}, u_{2} u_{4} u_{8}$ and $u_{2} u_{5} u_{6} \in K$. Then $\operatorname{Lk}\left(u_{5}\right)$ is $C_{5}\left(u_{7}, u_{4}, u_{9}, u_{6}, u_{2}\right)$, a contradiction. So, by considering $\operatorname{Lk}\left(u_{1}\right), u_{1} u_{3} u_{6}, u_{1} u_{6} u_{7}$ and $u_{1} u_{4} u_{7} \in K$.

Clearly, either $u_{3} u_{4}$ or $u_{4} u_{5} \in \operatorname{Lk}\left(u_{7}\right)$. If $u_{4} u_{5} u_{7} \in K$, then $u_{2} u_{3} u_{7}, u_{2} u_{7} u_{8}$ and $u_{3} u_{5} u_{7}$ are faces (if $u_{3} u_{7} u_{8} \in K$, then, to complete $\operatorname{Lk}\left(u_{3}\right), u_{3} u_{5} u_{8} \in K$, which implies 
$\left.\operatorname{deg}\left(u_{5}\right)<8\right) . \operatorname{Lk}\left(u_{5}\right)$ shows that $u_{2} u_{5} u_{6}$ and $u_{2} u_{5} u_{8}$ are faces. The link of $u_{8}$ now contains $C_{5}\left(u_{2}, u_{7}, u_{9}, u_{1}, u_{5}\right)$, a contradiction. So, $u_{3} u_{4} \in \operatorname{Lk}\left(u_{7}\right)$.

From the links of $u_{7}, u_{3}, u_{8}$ and $u_{2}, u_{3} u_{5} u_{7}, u_{2} u_{5} u_{7}, u_{2} u_{7} u_{8}, u_{2} u_{3} u_{8}, u_{3} u_{6} u_{8}$, $u_{4} u_{5} u_{8}, u_{4} u_{6} u_{8}, u_{2} u_{4} u_{6}$ and $u_{2} u_{5} u_{6}$ are faces. Here, $K$ is isomorphic, via the map $(1,3,5,7)(2,4,6,8)$, to $M_{2}$.

Subcase 2.2: $u_{1} u_{5} u_{6}$ is a face. To complete $\operatorname{Lk}\left(u_{1}\right), u_{1} u_{3} u_{6}, u_{1} u_{3} u_{7}$ and $u_{1} u_{4} u_{7}$ have to be faces. $\operatorname{Lk}\left(u_{5}\right)$ shows that the edge $u_{4} u_{5}$ belongs to either $u_{2} u_{4} u_{5}$ or $u_{4} u_{5} u_{7}$.

If $u_{4} u_{5} u_{7} \in K$, then, by considering $\operatorname{Lk}\left(u_{5}\right)$ and $\operatorname{Lk}\left(u_{7}\right), u_{2} u_{3} u_{5} \in K, u_{3} u_{5} u_{7} \notin K$ and hence $u_{2} u_{5} u_{7}$ and $u_{3} u_{5} u_{8} \in K$. To complete $\operatorname{Lk}\left(u_{3}\right), u_{3} u_{7} u_{8}$ and $u_{3} u_{4} u_{6}$ have to be faces (since $\operatorname{Lk}\left(u_{4}\right)$ shows that $u_{3} u_{4} u_{7} \notin K$ ). This implies that the link of $u_{8}$ contains $C_{5}\left(u_{3}, u_{7}, u_{9}, u_{1}, u_{5}\right)$, a contradiction. So, $u_{2} u_{4} u_{5} \in K$.

The links of $u_{5}, u_{2}, u_{8}$ and $u_{4}$ show that $u_{3} u_{5} u_{7}, u_{2} u_{5} u_{7}, u_{3} u_{5} u_{8}, u_{2} u_{6} u_{8}$ and $u_{4} u_{6} u_{8}$ are faces. Then, from $\operatorname{Lk}\left(u_{2}\right)$, either $u_{2} u_{3} u_{6} \in K$ or $u_{2} u_{3} u_{8} \in K$.

In the first case the links of $u_{2}, u_{6}$ and $u_{8}$ show that $u_{2} u_{7} u_{8}, u_{4} u_{6} u_{7}$ and $u_{3} u_{4} u_{8}$ are faces. Here, $K$ is isomorphic, via the map $(1,7,2,5,8,4,3,6,9)$, to $M_{2}$.

In the second case the links of $u_{2}, u_{6}$ and $u_{8}$ show that $u_{2} u_{6} u_{7}, u_{3} u_{4} u_{6}$ and $u_{4} u_{7} u_{8}$ are faces. Here, $K$ is isomorphic, via the map $(1,2,7,5,3)(4,8)$, to $M_{1}$.

Subcase 2.3: $u_{1} u_{5} u_{7}$ is a face. From $\operatorname{Lk}\left(u_{1}\right), u_{1} u_{3} u_{6}$ and one of $u_{1} u_{3} u_{4}$ or $u_{1} u_{4} u_{6}$ are faces.

If $u_{1} u_{3} u_{4} \in K$, then so is $u_{1} u_{6} u_{7}$. The links of $u_{3}, u_{6}$ and $u_{4}$ show that $u_{3} u_{5} u_{6}$, $u_{2} u_{3} u_{6} \notin K, u_{2} u_{4} u_{6}, u_{4} u_{6} u_{8}, u_{3} u_{6} u_{8}, u_{4} u_{5} u_{7}$ and $u_{4} u_{7} u_{8}$ are faces. This shows that the link of $u_{7}$ contains $C_{6}\left(u_{4}, u_{5}, u_{1}, u_{6}, u_{9}, u_{8}\right)$, a contradiction. So, $u_{1} u_{4} u_{6}$ is a face.

Clearly, $u_{1} u_{3} u_{7} \in K$. We now observe that either $u_{3} u_{4} u_{7}$ or $u_{3} u_{4} u_{8}$ is a face (since, $\operatorname{Lk}\left(u_{6}\right)$ shows that $\left.u_{3} u_{4} u_{6} \notin K\right)$.

In the first case the links of $u_{3}, u_{4}, u_{5}$ and $u_{6}$ show that $u_{3} u_{5} u_{8} \in K, u_{4} u_{5} u_{7} \notin K$ and hence $u_{2} u_{5} u_{7}, u_{2} u_{4} u_{5}, u_{3} u_{5} u_{6}, u_{2} u_{3} u_{8}, u_{4} u_{6} u_{8}, u_{4} u_{7} u_{8}, u_{2} u_{6} u_{8}$ and $u_{2} u_{6} u_{7}$ are faces. Here, $K$ is isomorphic, via the map $(1,4,6,3,9,7,5,2,8)$, to $M_{2}$.

In the second case $u_{4} u_{5} u_{7}$ has to be a face (if $u_{2} u_{4} u_{5} \in K$, then, from $\operatorname{Lk}\left(u_{4}\right)$ and $\left.\operatorname{Lk}\left(u_{7}\right), \operatorname{deg}\left(u_{7}\right)<8\right)$. Considering the links of $u_{5}, u_{3}, u_{7}$ and $u_{6}, u_{2} u_{3} u_{5} \in K$, $u_{3} u_{5} u_{8} \notin K$ and $u_{2} u_{5} u_{8}, u_{3} u_{5} u_{6}, u_{3} u_{7} u_{8}, u_{2} u_{4} u_{7}, u_{2} u_{6} u_{7}, u_{2} u_{6} u_{8}$ and $u_{4} u_{6} u_{8}$ are faces. Here, $K$ is isomorphic, via the map $(1,3,6,4,9,7)(5,8)$, to $M_{2}$.

Case 3: $u_{1} u_{6} u_{8}$ is a face. Clearly, $u_{3} u_{6}, u_{5} u_{6}$ or $u_{6} u_{7} \in \operatorname{Lk}\left(u_{1}\right)$.

If $u_{1} u_{6} u_{7} \in K$, then $u_{1} u_{3} u_{5} \in K$. The links of $u_{6}, u_{2}$ and $u_{1}$ show that $u_{2} u_{4} u_{6}$, $u_{3} u_{4} u_{6}, u_{1} u_{3} u_{7}$ and $u_{1} u_{4} u_{5}$ are faces. Here, $\operatorname{Lk}\left(u_{4}\right)$ contains $C_{6}\left(u_{6}, u_{3}, u_{9}, u_{5}, u_{1}, u_{2}\right)$, a contradiction.

If $u_{1} u_{5} u_{6} \in K$, then $u_{1} u_{3} u_{7} \in K$. It is clear from $\operatorname{Lk}\left(u_{6}\right)$ that $u_{2} u_{4} u_{6}$ and $u_{3} u_{4} u_{6}$ are faces. Considering the links of $u_{1}, u_{4}, u_{8}$ and $u_{5}, u_{1} u_{4} u_{7}, u_{1} u_{3} u_{5}, u_{4} u_{5} u_{8}, u_{4} u_{7} u_{8}$, $u_{3} u_{5} u_{7} \in K$, a contradiction. So, $u_{3} u_{6} \in \operatorname{Lk}\left(u_{1}\right)$.

Clearly, $u_{5} u_{7} \in \operatorname{Lk}\left(u_{1}\right)$. Now, either $u_{1} u_{4} u_{5}$ or $u_{1} u_{4} u_{7} \in K$.

If $u_{1} u_{4} u_{5} \in K$, then $u_{1} u_{3} u_{7} \in K$. The links of $u_{4}, u_{8}, u_{6}$ and $u_{3}$ show that $u_{4} u_{6} u_{8}$, $u_{4} u_{6} u_{7}, u_{2} u_{5} u_{6}, u_{2} u_{3} u_{6}$ and $u_{3} u_{5} u_{7}$ which imply $\operatorname{deg}\left(u_{7}\right)<8$. So, $u_{1} u_{4} u_{7}$ is a face.

The link of $u_{1}$ shows that $u_{1} u_{3} u_{5}$ is a face. Now, $u_{4} u_{5} u_{8}\left(\neq u_{4} u_{5} u_{9}\right)$ is the face having $u_{4} u_{5}$ as an edge. (If $u_{2} u_{4} u_{5} \in K$, considering the links of $u_{4}, u_{7}$ and $u_{2}$, we observe that $u_{3} u_{5} \in \operatorname{Lk}\left(u_{7}\right)$, a contradiction. $\operatorname{Lk}\left(u_{7}\right)$ shows that $u_{4} u_{5} u_{7} \notin K$.) The links of $u_{3}$ and $u_{5}$ 
show that $u_{2} u_{5} u_{6}$ is a face. To complete $\operatorname{Lk}\left(u_{5}\right), u_{2} u_{5} u_{7}$ and $u_{3} u_{5} u_{8}$ have to be faces (if $u_{2} u_{3} u_{5} \in K$, then $u_{2} u_{3} \in \operatorname{Lk}\left(u_{8}\right)$, a contradiction). Now, the second face through $u_{6} u_{8}$ is $u_{2} u_{6} u_{8}$ or $u_{4} u_{6} u_{8}$.

In the first case the links of $u_{6}, u_{4}$ and $u_{7}$ show that $u_{3} u_{4} u_{6}, u_{4} u_{6} u_{7}, u_{2} u_{4} u_{8}, u_{2} u_{3} u_{7}$ and $u_{3} u_{7} u_{8}$ are faces. Here, $K$ is isomorphic, via the map $(2,5)(3,9,6,8)(4,7)$, to $M_{2}$.

In the second case the links of $u_{8}, u_{7}$ and $u_{6}$ show that $u_{2} u_{3} u_{8}, u_{2} u_{7} u_{8}, u_{3} u_{4} u_{7}, u_{3} u_{6} u_{7}$ and $u_{2} u_{4} u_{6}$ are faces. Here, $K$ is isomorphic, via the map $(2,7,3)$, to $M_{1}$.

The theorem now follows from Lemma 4.

Proof of Theorem 6 . Let $K$ be a 10 -vertex neighbourly simplicial equivelar polyhedron. Choose a vertex, say $u$, and let the link of $u$ be $C_{9}\left(u_{1}, \ldots, u_{9}\right)$.

Case 1: There exists no face of the form $u_{i} u_{i+1} u_{i+3}$ or $u_{i} u_{i+1} u_{i+7}$. If $u_{i} u_{i+1} u_{i+5}$ is a face for each $i \in\{1, \ldots, 9\}$ (additions in the subscript are modulo 9), then $u_{1} u_{2} u_{6}, u_{1} u_{5} u_{6}$ and $u_{1} u_{5} u_{9} \in K$. This implies that $C_{5}\left(u_{2}, u_{6}, u_{5}, u_{9}, u\right)$ is in $\operatorname{Lk}\left(u_{1}\right)$, a contradiction. So assume, without loss, that $u_{1} u_{2} u_{5} \in K$.

Claim 1. $u_{1} u_{4} u_{9}$ is a face.

Since $u_{1} u_{8} u_{9}$ is not a face, the second face through $u_{1} u_{9}$ is $u_{1} u_{4} u_{9}, u_{1} u_{6} u_{9}$ or $u_{1} u_{7} u_{9}$. However, by the assumption, $u_{1} u_{7} u_{9}$ is not a face.

If $u_{1} u_{6} u_{9} \in K$, then $u_{1} u_{5}$ is in one of $u_{1} u_{3} u_{5}, u_{1} u_{4} u_{5}, u_{1} u_{5} u_{7}$ or $u_{1} u_{5} u_{8}$.

If $u_{1} u_{3} u_{5} \in K$, then $u_{1} u_{4} u_{7}$ and $u_{1} u_{4} u_{8} \in K$ (since $u_{3} u_{4}$ and $u_{7} u_{8} \notin \operatorname{Lk}\left(u_{1}\right)$ ). The edge $u_{5} u_{6}$ belongs to either $u_{2} u_{5} u_{6}$ or $u_{5} u_{6} u_{9}$. If $u_{2} u_{5} u_{6} \in K$, the links of $u_{5}, u_{1}, u_{3}$ and $u_{6}$ show that $u_{4} u_{5} u_{8}, u_{5} u_{8} u_{9}, u_{3} u_{5} u_{7}, u_{1} u_{3} u_{8}, u_{1} u_{6} u_{7}, u_{3} u_{4} u_{9}, u_{4} u_{6} u_{9}, u_{2} u_{4} u_{6}$ and $u_{2} u_{4} u_{7} \in K$. The link of $u_{6}$ contains $C_{7}\left(u_{2}, u_{5}, u, u_{7}, u_{1}, u_{9}, u_{4}\right)$, a contradiction. If $u_{5} u_{6} u_{9} \in K$, then the links of $u_{6}, u_{1}$ and $u_{8}$ imply $u_{1} u_{7} \notin \operatorname{Lk}\left(u_{6}\right), u_{1} u_{3} u_{7}, u_{1} u_{6} u_{8} \in K$, $u_{3} u_{4} u_{6}, u_{4} u_{6} u_{8} \notin K$ and hence $u_{4} u_{6} u_{7} \in K$, a contradiction to the assumption.

If $u_{1} u_{4} u_{5} \in K$, then, by considering the links of $u_{5}, u_{1}, u_{6}, u_{4}$ and $u_{3}, u_{1} u_{3} u_{7}, u_{1} u_{3} u_{8}$, $u_{5} u_{6} u_{9}, u_{1} u_{4} u_{7}, u_{1} u_{6} u_{8}, u_{4} u_{6} u_{8}, u_{2} u_{4} u_{6}, u_{2} u_{3} u_{6}, u_{3} u_{6} u_{7}$ (since $u_{3} u_{4}, u_{3} u_{8} \notin \operatorname{Lk}\left(u_{6}\right)$ ), $u_{3} u_{4} u_{9}, u_{4} u_{7} u_{9}, u_{4} u_{7} u_{8}, u_{3} u_{5} u_{8}$ and $u_{5} u_{7} u_{8}$ are faces. This gives a contradiction to the assumption.

If $u_{1} u_{5} u_{7} \in K$, then $u_{1} u_{3} u_{8}$ and $u_{1} u_{4} u_{8} \in K$. If $u_{2} u_{5} u_{6} \in K$, we see that $u_{8} u_{9} \in$ $\operatorname{Lk}\left(u_{5}\right)$ (if not, $\operatorname{Lk}\left(u_{5}\right)$ shows that $u_{3} u_{5} u_{8}$ and $u_{4} u_{5} u_{8} \in K$ which implies that $C_{4}\left(u_{3}, u_{1}\right.$, $\left.u_{4}, u_{5}\right)$ is in $\left.\operatorname{Lk}\left(u_{8}\right)\right)$. Hence, the links of $u_{5}, u_{1}, u_{6}, u_{7}$ and $u_{9}$ show that $u_{3} u_{5} u_{7}, u_{1} u_{4} u_{7}$, $u_{1} u_{3} u_{6}, u_{2} u_{6} u_{8}, u_{4} u_{6} u_{8}, u_{4} u_{6} u_{9}, u_{4} u_{7} u_{9}, u_{2} u_{7} u_{9}, u_{2} u_{7} u_{8}, u_{2} u_{3} u_{9}, u_{3} u_{5} u_{9}$ and $u_{4} u_{5} u_{8}$ are faces. Here, $u_{4} u_{8}$ is an edge in $u_{1} u_{4} u_{8}, u_{4} u_{5} u_{8}$ and $u_{4} u_{6} u_{8}$, a contradiction. If $u_{5} u_{6} u_{9} \in K$, then $u_{4} u_{8} \in \operatorname{Lk}\left(u_{5}\right), u_{3} u_{5} \notin \operatorname{Lk}\left(u_{8}\right)$. The links of $u_{5}, u_{1}$ and $u_{9}$ show that $u_{3} u_{5} u_{7}, u_{3} u_{5} u_{9}, u_{1} u_{4} u_{7}, u_{1} u_{3} u_{6}, u_{3} u_{4} u_{9}$ and $u_{4} u_{6} u_{9} \in K$. Here $u_{6} u_{9}$ is an edge in $u_{1} u_{6} u_{9}, u_{5} u_{6} u_{9}$ and $u_{4} u_{6} u_{9}$, a contradiction.

Finally, let $u_{1} u_{5} u_{8} \in K$. Then $u_{1} u_{3} u_{7}, u_{1} u_{4} u_{7} \in K$. If $u_{2} u_{5} u_{6} \in K$, then the links of $u_{5}, u_{1}, u_{6}, u_{9}$ and $u_{2}$ show that $u_{4} u_{5} u_{9}, u_{5} u_{7} u_{9}, u_{3} u_{5} u_{7}, u_{3} u_{5} u_{8}, u_{1} u_{4} u_{8}, u_{1} u_{3} u_{6}$, $u_{3} u_{6} u_{7}, u_{4} u_{6} u_{9}, u_{4} u_{6} u_{8}, u_{2} u_{6} u_{8}, u_{3} u_{8} u_{9}, u_{2} u_{3} u_{9}, u_{2} u_{7} u_{9}$ and $u_{2} u_{4} u_{8} \in K$. Hence $\operatorname{Lk}\left(u_{8}\right)$ contains $C_{3}\left(u_{2}, u_{6}, u_{4}\right)$, a contradiction. If $u_{5} u_{6} u_{9} \in K$, then $\operatorname{Lk}\left(u_{5}\right)$ shows that $u_{2} u_{4} u_{5}, u_{3} u_{5} u_{7}, u_{3} u_{5} u_{8}$ and $u_{5} u_{8} u_{9} \in K$. Hence $\operatorname{Lk}\left(u_{9}\right)$ contains $C_{5}\left(u_{5}, u_{8}, u, u_{1}, u_{6}\right)$, a contradiction. This proves Claim 1. 
Clearly, the second face through $u_{1} u_{4}$ is $u_{1} u_{4} u_{6}, u_{1} u_{4} u_{7}$ or $u_{1} u_{4} u_{8}$.

Claim 2. $u_{1} u_{4} u_{7}$ is a face.

If $u_{1} u_{4} u_{6} \in K$, then $u_{1} u_{3} u_{7}, u_{1} u_{3} u_{8}$ are faces. We see that $u_{4} u_{5}$ is an edge in either $u_{4} u_{5} u_{8}$ or $u_{4} u_{5} u_{9}$. If $u_{4} u_{5} u_{9} \in K$, then the links of $u_{5}, u_{1}$ and $u_{4}$ imply that $u_{2} u_{5} u_{6}$, $u_{3} u_{5} u_{7}, u_{3} u_{5} u_{8}, u_{1} u_{5} u_{8}, u_{5} u_{7} u_{9}, u_{1} u_{6} u_{7}, u_{3} u_{4} u_{7}$ and $u_{2} u_{4} u_{7} \in K$. This implies $u_{3} u_{7}$ is in $u_{1} u_{3} u_{7}, u_{3} u_{5} u_{7}$ and $u_{3} u_{4} u_{7}$, a contradiction. Hence, $u_{4} u_{5} u_{8} \in K$ and either $u_{1} u_{5} u_{8}$ or $u_{1} u_{5} u_{7} \in K$. In the former case the links of $u_{1}, u_{4}$ and $u_{6}$ show that $u_{1} u_{6} u_{8} \in K$, $u_{4} u_{6} u_{8}$ and $u_{4} u_{6} u_{9} \notin K$ and hence $u_{2} u_{4} u_{6}$ is a face. It is clear that $u_{1} u_{6} u_{8}$ is the only face having $u_{6} u_{8}$ as an edge, a contradiction. In the latter case the links of $u_{1}, u_{5}, u_{7}$ and $u_{4}$ show that $u_{1} u_{6} u_{7}, u_{2} u_{5} u_{7}, u_{3} u_{5} u_{7}, u_{3} u_{5} u_{9}, u_{2} u_{7} u_{9}, u_{4} u_{7} u_{9}, u_{4} u_{8} u_{9}$ and $u_{3} u_{4} u_{5} \in K$, a contradiction.

If $u_{1} u_{4} u_{8} \in K$, either $u_{4} u_{5} u_{9}$ or $u_{4} u_{5} u_{8} \in K$. In either case $\operatorname{Lk}\left(u_{4}\right)$ implies that $u_{3} u_{4} u_{7}, u_{2} u_{4} u_{7}$ and $u_{2} u_{4} u_{6}$ are faces. Hence $u_{1} u_{6} u_{7} \in K$. To complete $\operatorname{Lk}\left(u_{1}\right)$, either $u_{1} u_{3} u_{8}$ or $u_{1} u_{6} u_{8} \in K$. If $u_{1} u_{3} u_{8} \in K$, then to complete $\operatorname{Lk}\left(u_{6}\right)$, either $u_{6} u_{8} u_{9}$ or $u_{5} u_{6} u_{8} \in K$, a contradiction. Hence $u_{1} u_{6} u_{8}$ and therefore $u_{1} u_{3} u_{5}$ and $u_{1} u_{3} u_{7} \in K$. Clearly, $u_{2} u_{7} u_{8} \in K$. This implies $\operatorname{deg}\left(u_{7}\right)<9$, a contradiction. This proves Claim 2 .

Again, either $u_{4} u_{5} u_{9}$ or $u_{4} u_{5} u_{8} \in K$. If $u_{4} u_{5} u_{9} \in K$, then it is clear from $\operatorname{Lk}\left(u_{4}\right)$ that $u_{3} u_{4} u_{8}, u_{4} u_{6} u_{8}, u_{2} u_{4} u_{6}$ and $u_{2} u_{4} u_{7} \in K$. It is easy to see that $u_{3} u_{6} u_{7}, u_{2} u_{5} u_{6}, u_{2} u_{7} u_{8}$, $u_{2} u_{3} u_{9}$ and $u_{5} u_{8} u_{9} \in K$. Hence $\operatorname{deg}\left(u_{9}\right)<9$, a contradiction. Thus, $u_{4} u_{5} u_{8} \in K$.

Clearly, $u_{3} u_{4} u_{7}$ or $u_{3} u_{4} u_{9}$ is a face through $u_{3} u_{4}$. If $u_{3} u_{4} u_{9} \in K$, the links of $u_{4}, u_{1}$ and $u_{6}$ show that $u_{2} u_{4} u_{7}, u_{4} u_{6} u_{8}, u_{1} u_{3} u_{7}, u_{1} u_{6} u_{8}$ and $u_{3} u_{6} u_{7} \in K$. To complete $\operatorname{Lk}\left(u_{7}\right)$, either $u_{5} u_{7} u_{8}$ or $u_{7} u_{8} u_{9} \in K$, a contradiction. So, $u_{3} u_{4} u_{7} \in K$.

The links of $u_{4}, u_{1}$ and $u_{7}$ show that $u_{1} u_{6} u_{7}, u_{1} u_{3} u_{8}, u_{2} u_{4} u_{6}, u_{2} u_{7} u_{8}$ and $u_{5} u_{7} u_{9}$ are faces.

Now, either $u_{2} u_{5} u_{8}$ or $u_{5} u_{8} u_{9} \in K$. If $u_{5} u_{8} u_{9} \in K$, the links of $u_{5}, u_{1}, u_{4}, u_{6}$ show that $C_{3}\left(u_{3}, u_{1}, u_{6}\right)$ is in $\operatorname{Lk}\left(u_{8}\right)$, a contradiction. So, $u_{2} u_{5} u_{8} \in K$.

Now, the links of $u_{1}, u_{5}, u_{6}, u_{8}$ and $u_{2}$ show that $u_{1} u_{3} u_{5}, u_{1} u_{6} u_{8}, u_{3} u_{5} u_{7}, u_{5} u_{6} u_{9}$, $u_{4} u_{6} u_{8}, u_{2} u_{3} u_{6}, u_{3} u_{6} u_{9}, u_{3} u_{8} u_{9}, u_{2} u_{7} u_{9}$ and $u_{2} u_{4} u_{9}$ are faces. Here, $K$ is $N_{1}$.

Case 2: There exists a face of the form $u_{i} u_{i+1} u_{i+3}$ or $u_{i} u_{i+1} u_{i+7}$. We can assume without loss that $u_{1} u_{2} u_{4} \in K$. From $\operatorname{Lk}\left(u_{1}\right)$ we see that $u_{1} u_{9}$ is an edge in one of $u_{1} u_{3} u_{9}, u_{1} u_{5} u_{9}$, $u_{1} u_{6} u_{9}$ or $u_{1} u_{7} u_{9}$.

Subcase 2.1: $u_{1} u_{7} u_{9} \in K$. The edge $u_{1} u_{4}$ belongs to one of $u_{1} u_{3} u_{4}, u_{1} u_{4} u_{5}, u_{1} u_{4} u_{6}$ or $u_{1} u_{4} u_{8}$.

2.1.1: $u_{1} u_{3} u_{4} \in K$. From $\operatorname{Lk}\left(u_{1}\right)$ we see that one of $u_{1} u_{5} u_{7}, u_{1} u_{6} u_{7}$ or $u_{1} u_{7} u_{8} \in K$.

If $u_{1} u_{6} u_{7} \in K$, we see from $\operatorname{Lk}\left(u_{1}\right)$ and $\operatorname{Lk}\left(u_{6}\right)$ that $u_{1} u_{6} u_{8}, u_{1} u_{5} u_{8}$ and $u_{1} u_{3} u_{5} \in K$. The link of $u_{4}$ shows that one of $u_{4} u_{5} u_{7}, u_{4} u_{5} u_{8}$ or $u_{4} u_{5} u_{9} \in K$.

If $u_{4} u_{5} u_{7}$ is a face, the links of $u_{4}$ and $u_{7}$ show that either $u_{4} u_{7} u_{8}$ or $u_{4} u_{7} u_{9} \in K$. In both cases, after completing $\operatorname{Lk}\left(u_{4}\right)$, we see from $\operatorname{Lk}\left(u_{6}\right)$ and $\operatorname{Lk}\left(u_{7}\right)$ that $u_{2} u_{3} u_{6}$ and $u_{2} u_{3} u_{7}$ are faces, a contradiction (since $u u_{2} u_{3}$ is already a face).

If $u_{4} u_{5} u_{8}$ is a face, then it is clear that $u_{4} u_{6} \notin \operatorname{Lk}\left(u_{8}\right)$ and $u_{4} u_{8} u_{9} \notin K$ (since, if $u_{4} u_{8} u_{9} \in K$, then $\operatorname{Lk}\left(u_{4}\right)$ implies that $u_{4} u_{6} u_{7} \in K$, a contradiction). The links of $u_{4}$ and $u_{7}$ show that $u_{4} u_{7} u_{8}$ and $u_{4} u_{7} u_{9} \in K$, which imply that $C_{6}\left(u_{9}, u_{1}, u_{6}, u, u_{8}, u_{4}\right)$ is in $\operatorname{Lk}\left(u_{7}\right)$. So, $u_{4} u_{5} u_{9} \in K$. 
Since $u_{6} u_{7} \notin \operatorname{Lk}\left(u_{4}\right)$, we see on completing $\operatorname{Lk}\left(u_{4}\right)$ that $u_{4} u_{6} u_{8}$ and $u_{4} u_{7} u_{8} \in K$. The links of $u_{7}, u_{4}, u_{8}$ and $u_{6}$ show that $u_{4} u_{6} u_{9}, u_{2} u_{4} u_{7}, u_{2} u_{3} u_{8}$ and $u_{2} u_{3} u_{6} \in K$, a contradiction. Therefore, either $u_{1} u_{5} u_{7}$ or $u_{1} u_{7} u_{8} \in K$.

2.1.1.1: $u_{1} u_{5} u_{7} \in K$. The link of $u_{1}$ shows that either $u_{1} u_{5} u_{6}$ or $u_{1} u_{5} u_{8}$ is a face.

2.1.1.1.1: $u_{1} u_{5} u_{6}$ is a face. From $\operatorname{Lk}\left(u_{1}\right)$ we see that $u_{1} u_{6} u_{8}, u_{1} u_{3} u_{8} \in K$. Considering the links of $u_{4}$ and $u_{5}$, either $u_{4} u_{5} u_{8}$ or $u_{4} u_{5} u_{9} \in K$.

Subcase A: $u_{4} u_{5} u_{8}$ is a face. The links of $u_{5}$ and $u_{7}$ show that either $u_{2} u_{5} u_{7}$ or $u_{3} u_{5} u_{7} \in K$.

A.1: $u_{2} u_{5} u_{7}$ is a face. If $u_{2} u_{3} u_{5} \in K, \operatorname{Lk}\left(u_{5}\right), \operatorname{Lk}\left(u_{3}\right)$ and $\operatorname{Lk}\left(u_{8}\right)$ show that $u_{3} u_{5} u_{9}$, $u_{3} u_{6} u_{7}$ and $u_{3} u_{6} u_{8}$ are faces. This implies that $C_{3}\left(u_{3}, u_{1}, u_{6}\right)$ is in $\operatorname{Lk}\left(u_{8}\right)$.

To complete $\operatorname{Lk}\left(u_{5}\right), u_{2} u_{5} u_{9}, u_{3} u_{5} u_{8}$ and $u_{3} u_{5} u_{9} \in K$. The links of $u_{3}$ and $u_{6}$ show that $u_{3} u_{6} u_{7}, u_{4} u_{6} u_{9}$ and $u_{2} u_{4} u_{6} \in K$ (since, $u_{4} u_{6} \notin \mathrm{L}\left(u_{8}\right)$ and $\operatorname{Lk}\left(u_{3}\right)$ ). The links of $u_{4}, u_{8}, u_{7}$ and $u_{6}$ show that $u_{4} u_{7} u_{9}, u_{4} u_{7} u_{8}, u_{2} u_{6} u_{8}, u_{2} u_{8} u_{9}, u_{2} u_{3} u_{7}$ and $u_{3} u_{6} u_{9}$ are faces. In this case $K$ is $N_{11}$ (more precisely, $K$ is isomorphic to $N_{11}$ by the map $\varphi$, where $\varphi(u)=0$ and $\varphi\left(u_{i}\right)=i$, for $\left.1 \leq i \leq 9\right)$.

A.2: $u_{3} u_{5} u_{7} \in K$. If $u_{2} u_{3} u_{5} \in K$, the links of $u_{5}$ and $u_{3}$ show that $u_{5} u_{8} u_{9}$ and $u_{3} u_{8} u_{9} \in K$ (since $\left.u_{3} u_{6} \notin \mathrm{L}\left(u_{8}\right)\right)$, a contradiction. Hence, $u_{2} u_{5} u_{9}, u_{2} u_{5} u_{8}$ and $u_{3} u_{5} u_{9}$ are the faces required to complete $\operatorname{Lk}\left(u_{5}\right)$. If $u_{2} u_{3} u_{7} \in K, \operatorname{Lk}\left(u_{3}\right)$ shows that $u_{3} u_{5} u_{6} \in K$, a contradiction.

We now observe from the links of $u_{3}, u_{5}$ and $u_{9}$ that the edge $u_{2} u_{3}$ belongs to $u_{2} u_{3} u_{6}$. The edge $u_{3} u_{6}$ is in either $u_{3} u_{6} u_{7}$ or $u_{3} u_{6} u_{9}$.

In the first case the links of $u_{3}, u_{7}, u_{4}$ and $u_{2}$ show that $u_{3} u_{8} u_{9}, u_{2} u_{4} u_{7}, u_{4} u_{7} u_{9}$, $u_{4} u_{6} u_{9}, u_{4} u_{6} u_{8}, u_{2} u_{7} u_{8}$ and $u_{2} u_{6} u_{9}$ are faces. Here, $K$ is $N_{7}$.

In the second case the links of $u_{3}, u_{7}, u_{2}$ and $u_{4}$ show that $u_{3} u_{7} u_{8}, u_{2} u_{4} u_{7}, u_{4} u_{6} u_{7}$, $u_{2} u_{7} u_{9}, u_{2} u_{6} u_{8}, u_{4} u_{6} u_{9}$ and $u_{4} u_{8} u_{9}$ are faces. Now, $K$ is $N_{12}$.

Subcase B: $u_{4} u_{5} u_{9} \in K$. If $u_{2} u_{4} u_{8} \in K$, the links of $u_{4}$ and $u_{7}$ show that $u_{4} u_{6} u_{7}$, $u_{4} u_{6} u_{8}$ and $u_{4} u_{7} u_{9} \in K$. Here $C_{6}\left(u_{8}, u_{4}, u_{7}, u, u_{5}, u_{1}\right)$ is in $\operatorname{Lk}\left(u_{6}\right)$. We now observe from $\operatorname{Lk}\left(u_{4}\right)$ that either $u_{2} u_{4} u_{6}$ or $u_{2} u_{4} u_{7} \in K$.

In the first case the links of $u_{4}, u_{8}, u_{7}, u_{6}$ and $u_{9}$ show that $u_{4} u_{6} u_{8}, u_{4} u_{7} u_{8}, u_{4} u_{7} u_{9}$, $u_{2} u_{5} u_{8}, u_{2} u_{3} u_{7}, u_{3} u_{5} u_{8} u_{2} u_{8} u_{9}, u_{3} u_{6} u_{7}, u_{3} u_{6} u_{9}, u_{2} u_{6} u_{9}, u_{2} u_{5} u_{7}$ and $u_{3} u_{5} u_{9}$ are faces. Now, $K$ is $N_{14}$.

In the second case the links of $u_{4}, u_{6}, u_{7}, u_{2}$ and $u_{3}$ show that $u_{4} u_{6} u_{8}, u_{4} u_{7} u_{8}, u_{4} u_{6} u_{9}$, $u_{2} u_{3} u_{6}, u_{3} u_{6} u_{7}, u_{2} u_{6} u_{9}, u_{2} u_{5} u_{7}, u_{3} u_{7} u_{9}, u_{2} u_{5} u_{8}, u_{2} u_{8} u_{9}, u_{3} u_{5} u_{9}$ and $u_{3} u_{5} u_{8}$ are faces. Here, $K$ is $N_{8}$.

2.1.1.1.2: $u_{1} u_{5} u_{8} \in K$. The links of $u_{1}$ and $u_{4}$ show that $u_{1} u_{6} u_{8}, u_{1} u_{3} u_{6} \in K$ and one of $u_{4} u_{5} u_{7}, u_{4} u_{5} u_{8}$ or $u_{4} u_{5} u_{9} \in K$.

Subcase A: $u_{4} u_{5} u_{7} \in K$. If $u_{2} u_{4} u_{6} \in K$, then $\operatorname{Lk}\left(u_{4}\right)$ and $\operatorname{Lk}\left(u_{8}\right)$ show that $u_{4} u_{7} u_{9}$ and $u_{4} u_{8} u_{9} \in K$, thereby showing that $\operatorname{deg}\left(u_{9}\right)<9$. In the case when $u_{2} u_{4} u_{9} \in K$, $\operatorname{Lk}\left(u_{4}\right)$ and $\operatorname{Lk}\left(u_{7}\right)$ show that $u_{4} u_{6} u_{8}$ and $u_{2} u_{3} u_{7}$ are faces (since, from $\operatorname{Lk}\left(u_{4}\right)$, either $u_{4} u_{7} u_{8}$ or $\left.u_{4} u_{6} u_{7} \in K\right)$. Now, the links of $u_{5}$ and $u_{2}$ show that $u_{2} u_{5} u_{9}, u_{3} u_{5} u_{9}$ and $u_{2} u_{6} u_{8}$ are faces. This implies that $u_{6} u_{8}$ is an edge in $u_{1} u_{6} u_{8}, u_{2} u_{6} u_{8}$ and $u_{4} u_{6} u_{8}$, a contradiction. Thus, from $\operatorname{Lk}\left(u_{4}\right), u_{2} u_{4} u_{8} \in K$.

The links of $u_{4}, u_{7}, u_{8}, u_{2}$ and $u_{9}$ show that $u_{4} u_{6} u_{9}, u_{4} u_{6} u_{7}, u_{4} u_{8} u_{9}, u_{2} u_{3} u_{7}$, $u_{2} u_{7} u_{9}, u_{3} u_{7} u_{8}, u_{2} u_{5} u_{6}, u_{3} u_{5} u_{9}, u_{2} u_{5} u_{9}, u_{3} u_{6} u_{9}, u_{2} u_{6} u_{8}$ and $u_{3} u_{5} u_{8}$ are faces. Here, $\mathrm{K}$ is $\mathrm{N}_{5}$. 
Subcase B: $u_{4} u_{5} u_{8} \in K$. It is clear that $C_{4}\left(u_{4}, u_{5}, u_{1}, u_{6}\right)$ is in $\operatorname{Lk}\left(u_{8}\right)$ if $u_{4} u_{6} u_{8} \in K$. If $u_{4} u_{7} u_{8} \in K$, considering the links of $u_{4}, u_{7}$ and $u_{8}$ we see that $u_{2} u_{3} u_{7}$ and $u_{2} u_{3} u_{8}$ are faces, a contradiction. Hence, from $\operatorname{Lk}\left(u_{4}\right), u_{4} u_{8} u_{9} \in K$. Now, on considering $\operatorname{Lk}\left(u_{9}\right)$ also, $u_{4} u_{6} u_{9}, u_{4} u_{6} u_{7}$ and $u_{2} u_{4} u_{7} \in K$. The links of $u_{8}, u_{2}$ and $u_{9}$ show that $u_{2} u_{3} u_{8}$, $u_{3} u_{7} u_{8}, u_{2} u_{6} u_{8}, u_{2} u_{5} u_{9}$ and $u_{3} u_{5} u_{9}$ are faces. To complete $\operatorname{Lk}\left(u_{5}\right)$, either $u_{2} u_{5} u_{6}$ or $u_{3} u_{5} u_{6}$ is a face.

In the first case the links of $u_{2}, u_{5}$ and $u_{3}$ show that $u_{2} u_{7} u_{9}, u_{3} u_{5} u_{7}$ and $u_{3} u_{6} u_{9}$ are faces. Clearly, $K$ is $N_{6}$.

In the second case the links of $u_{3}, u_{9}$ and $u_{5}$ show that $u_{3} u_{7} u_{9}, u_{2} u_{6} u_{9}$ and $u_{2} u_{5} u_{7}$ are faces and therefore $K$ is isomorphic, via the map $(0,4,2,3,1)(5,7,6,9)$, to $N_{7}$.

Subcase C: $u_{4} u_{5} u_{9} \in K$. Considering the link of $u_{4}$, we see that one of $u_{4} u_{6} u_{9}$, $u_{4} u_{7} u_{9}$ or $u_{4} u_{8} u_{9}$ is a face.

C.1: $u_{4} u_{6} u_{9} \in K$. The links of $u_{4}$ and $u_{8}$ show that $u_{4} u_{7} u_{8}, u_{2} u_{4} u_{7}, u_{4} u_{6} u_{8}$ and $u_{2} u_{3} u_{8} \in K$. If $u_{6} u_{7} u_{9} \in K, \operatorname{Lk}\left(u_{7}\right)$ shows that $u_{2} u_{3} u_{7} \in K$, a contradiction. Hence, we can conclude from $\operatorname{Lk}\left(u_{7}\right)$ that $u_{3} u_{6} u_{7} \in K$. From $\operatorname{Lk}\left(u_{6}\right)$ and $\operatorname{Lk}\left(u_{3}\right)$, we see that $u_{2} u_{6} u_{9}, u_{2} u_{5} u_{6}$ and $u_{3} u_{5} u_{9} \in K$. To complete $\operatorname{Lk}\left(u_{8}\right)$, either $u_{2} u_{8} u_{9}$ or $u_{3} u_{8} u_{9}$ is a face.

In the first case the links of $u_{8}, u_{2}$ and $u_{7}$ show that $u_{3} u_{5} u_{8}, u_{2} u_{5} u_{7}$ and $u_{3} u_{7} u_{9}$ are faces. Here, $K$ is isomorphic, via the map $(0,1,3,2,4)(5,9,8,6,7)$, to $N_{14}$.

In the second case the links of $u_{8}, u_{3}$ and $u_{2}$ show that $u_{2} u_{5} u_{8}, u_{3} u_{5} u_{7}$ and $u_{2} u_{7} u_{9}$ are faces. Here, $K$ is isomorphic, via the map, $(0,1,3,2,4)(5,9,8,6,7)$, to $N_{11}$.

C.2: $u_{4} u_{7} u_{9} \in K$. The link of $u_{4}$ shows that either $u_{4} u_{6} u_{7}$ or $u_{4} u_{7} u_{8} \in K$. In both cases $u_{2} u_{3} \in \operatorname{Lk}\left(u_{7}\right)$. If $u_{3} u_{5} u_{6} \in K$, the link of $u_{3}$ and $u_{8}$ show that $u_{3} u_{7} u_{9}$ and $u_{3} u_{8} u_{9} \in K$, a contradiction. Hence, $u_{2} u_{5} u_{6} \in K$ (the links of $u_{8}$ and $u_{5}$ show that $u_{5} u_{6} u_{8}$ and $\left.u_{5} u_{6} u_{9} \notin K\right)$. Considering the links of $u_{5}$ and $u_{9}$, we see that $u_{3} u_{5} u_{9}$, $u_{2} u_{6} u_{9}, u_{2} u_{8} u_{9}$ and $u_{3} u_{6} u_{9} \in K$. The links of $u_{6}, u_{4}, u_{2}$ and $u_{8}$ show that $u_{4} u_{6} u_{8}$, $u_{4} u_{6} u_{7}, u_{2} u_{4} u_{8}, u_{2} u_{5} u_{7}, u_{3} u_{7} u_{8}$ and $u_{3} u_{5} u_{8}$ are faces. In this case $K$ is isomorphic, via the map $(0,2,1,4,3)(5,7,9,6)$, to $N_{11}$.

C.3: $u_{4} u_{8} u_{9} \in K$. The links of $u_{4}, u_{6}, u_{3}, u_{8}, u_{2}$ and $u_{5}$ imply that $u_{4} u_{6} u_{8}, u_{4} u_{6} u_{7}$, $u_{2} u_{4} u_{7}, u_{2} u_{5} u_{6}, u_{2} u_{6} u_{9}, u_{3} u_{6} u_{9}, u_{2} u_{3} u_{8}, u_{2} u_{5} u_{8}, u_{2} u_{7} u_{9}, u_{3} u_{5} u_{7}, u_{3} u_{7} u_{8}$ and $u_{3} u_{5} u_{9}$ are faces. Here, $K$ is isomorphic, via the map $(0,2,1,4,3)(5,7,8,9,6)$, to $N_{14}$.

2.1.1.2: $u_{1} u_{7} u_{8} \in K$. The link of $u_{1}$ shows that either $u_{1} u_{5} u_{8}$ or $u_{1} u_{6} u_{8}$ is a face.

Claim 3. $u_{1} u_{5} u_{8}$ is a face.

If $u_{1} u_{6} u_{8} \in K$, the links of $u_{1}$ and $u_{4}$ show that $u_{1} u_{5} u_{6}, u_{1} u_{3} u_{5}$ and one of $u_{4} u_{5} u_{7}$, $u_{4} u_{5} u_{8}, u_{4} u_{5} u_{9} \in K$. If $u_{4} u_{5} u_{7} \in K$, from $\operatorname{Lk}\left(u_{4}\right)$, either $u_{4} u_{6} u_{7}$ or $u_{4} u_{6} u_{9} \in K$ (since $\left.u_{4} u_{7} u_{8} \notin K\right)$ ). In both the cases the links of $u_{2}, u_{4}, u_{6}$ and $u_{7}$ show that $u u_{2} u_{3}$, $u_{2} u_{3} u_{6}, u_{2} u_{3} u_{7} \in K$, a contradiction. If $u_{4} u_{5} u_{8} \in K, \operatorname{Lk}\left(u_{4}\right)$ and $\operatorname{Lk}\left(u_{8}\right)$ show that either $u_{4} u_{6} u_{8}$ or $u_{4} u_{8} u_{9} \in K$. Again, in both cases, considering $\operatorname{Lk}\left(u_{4}\right), \operatorname{Lk}\left(u_{9}\right)$ and $\operatorname{Lk}\left(u_{8}\right)$ we see that $u_{2} u_{3} u_{6}, u_{2} u_{3} u_{8} \in K$. So, $u_{4} u_{5} u_{9} \in K$. Since $u_{7} u_{8}$ already belongs to two faces, $u_{4} u_{7} u_{8} \notin K$. Hence, $u_{4} u_{6} u_{7}$ and $u_{4} u_{6} u_{8} \in K$, thereby showing that $C_{6}\left(u_{8}, u_{4}, u_{7}, u, u_{5}, u_{1}\right)$ is in $\operatorname{Lk}\left(u_{6}\right)$. This proves Claim 3.

The links of $u_{1}, u_{4}$ and $u_{5}$ show that $u_{1} u_{3} u_{6}, u_{1} u_{5} u_{6}$ and either $u_{4} u_{5} u_{7}$ or $u_{4} u_{5} u_{9} \in K$.

Subcase A: $u_{4} u_{5} u_{7} \in K$. Considering $\operatorname{Lk}\left(u_{4}\right)$, either $u_{4} u_{6} u_{7}$ or $u_{4} u_{7} u_{9} \in K$. In both cases $u_{2} u_{3} \in \operatorname{Lk}\left(u_{7}\right)$ and therefore, from $\operatorname{Lk}\left(u_{5}\right), u_{2} u_{5} u_{9}$ and $u_{3} u_{5} u_{9} \in K$. 
If $u_{4} u_{6} u_{7} \in K$, the links of $u_{4}, u_{6}, u_{8}, u_{3}$ and $u_{5}$ show that $u_{4} u_{8} u_{9}, u_{2} u_{6} u_{8}, u_{3} u_{6} u_{8}$, $u_{2} u_{6} u_{9}, u_{2} u_{4} u_{8}, u_{3} u_{5} u_{8}, u_{4} u_{6} u_{9}, u_{3} u_{7} u_{9}$ and $u_{2} u_{5} u_{7}$ are faces and, thus, $K$ is $N_{2}$.

If $u_{4} u_{7} u_{9} \in K$, the links of $u_{4}, u_{9}, u_{6}, u_{7}, u_{5}$ and $u_{8}$ show that $u_{2} u_{4} u_{8}, u_{4} u_{6} u_{8}, u_{4} u_{6} u_{9}$, $u_{3} u_{5} u_{7}, u_{2} u_{6} u_{7}, u_{2} u_{5} u_{8}, u_{3} u_{6} u_{8}, u_{3} u_{8} u_{9}$ and $u_{2} u_{6} u_{9}$ are faces. Here, $K$ is isomorphic, via the map $(0,4,2,3,1)(5,8,7,6,9)$, to $N_{5}$.

Subcase B: $u_{4} u_{5} u_{9} \in K$. The links of $u_{4}, u_{7}, u_{6}, u_{8}, u_{5}$ and $u_{9}$ show that $u_{4} u_{6} u_{8}$, $u_{4} u_{6} u_{7}, u_{2} u_{4} u_{7}, u_{4} u_{8} u_{9}, u_{3} u_{5} u_{7}, u_{3} u_{6} u_{9}, u_{2} u_{6} u_{9}, u_{2} u_{6} u_{8}, u_{2} u_{3} u_{8}, u_{3} u_{5} u_{8}, u_{2} u_{5} u_{7}$, $u_{2} u_{5} u_{9}$ and $u_{3} u_{7} u_{9} \in K$. In this case $K$ is isomorphic, via the map $(0,4,6,8,1)(2,5,9,3$, 7), to $N_{5}$.

\subsection{2: $u_{1} u_{4} u_{5} \in K$.}

Claim 4. For a vertex $x \neq u, u_{2}, u_{3}$ and $u_{4}$ cannot occur together in any order in the link of any of the vertices.

Let $x$ be any vertex in $K$. If $u_{2} u_{3} x$ and $u_{3} u_{4} x$ are faces, then $C_{4}\left(u_{2}, x, u_{4}, u\right)$ is in $\operatorname{Lk}\left(u_{3}\right)$. If $u_{2} u_{4} x$ and $u_{3} u_{4} x$ are faces, then $C_{6}\left(u_{3}, u, u_{5}, u_{1}, u_{2}, x\right)$ is in $\operatorname{Lk}\left(u_{4}\right)$ and finally if $u_{2} u_{3} x$ and $u_{2} u_{4} x$ are faces, then $C_{5}\left(u_{4}, u_{1}, u, u_{3}, x\right)$ is in $\operatorname{Lk}\left(u_{2}\right)$. Hence, Claim 4 is proved.

The links of $u_{1}$ and $u_{5}$ show that $u_{1} u_{3} u_{5}$ or $u_{1} u_{5} u_{8} \in K$.

Claim 5. $u_{1} u_{5} u_{8}$ is a face.

If $u_{1} u_{3} u_{5} \in K$, then $\operatorname{Lk}\left(u_{1}\right)$ and $\operatorname{Lk}\left(u_{5}\right)$ show that $u_{1} u_{6} u_{8}$ and one of $u_{2} u_{5} u_{6}, u_{5} u_{6} u_{8}$ or $u_{5} u_{6} u_{9} \in K$.

If $u_{2} u_{5} u_{6} \in K, \operatorname{Lk}\left(u_{5}\right)$ shows that $u_{5} u_{7} u_{8}$ and $u_{5} u_{7} u_{9} \in K$ (if $u_{5} u_{8} u_{9}$ and $u_{5} u_{7} u_{9}$ are faces, then $\left.\operatorname{deg}\left(u_{9}\right)<9\right)$. Hence $u_{7} u_{8} \notin \operatorname{Lk}\left(u_{1}\right)$ and therefore $u_{1} u_{6} u_{7}$ and $u_{1} u_{3} u_{8} \in K$. This shows that $C_{6}\left(u_{1}, u_{9}, u_{5}, u_{8}, u, u_{6}\right)$ is in $\operatorname{Lk}\left(u_{7}\right)$.

If $u_{5} u_{6} u_{8} \in K$, considering the links of $u_{6}$ and $u_{1}$, we see that $u_{1} u_{3} u_{6}$ and $u_{1} u_{7} u_{8}$ are faces, which imply that $u_{2}, u_{3}$ and $u_{4}$ are together in $\operatorname{Lk}\left(u_{8}\right)$, which is impossible from Claim 4. Thus, $u_{5} u_{6} u_{9} \in K$.

It is clear that $u_{1} u_{7} u_{8}$ and $u_{1} u_{3} u_{6}$ are faces (since $u_{1} u_{6} u_{7} \notin K$, from $\operatorname{Lk}\left(u_{6}\right)$ and Claim 4). Since $u_{7} u_{8} \notin \operatorname{Lk}\left(u_{5}\right), u_{2} u_{5} u_{7}$ and $u_{2} u_{5} u_{8}$ are faces. To complete $\operatorname{Lk}\left(u_{5}\right)$, either $u_{5} u_{7} u_{9}$ or $u_{5} u_{8} u_{9} \in K$, both of which are impossible by looking at $\operatorname{Lk}\left(u_{7}\right)$, $\operatorname{Lk}\left(u_{8}\right)$ and Claim 4. This proves Claim 5.

From Claim 5 and $\operatorname{Lk}\left(u_{1}\right), u_{1} u_{5} u_{8}$ and therefore $u_{1} u_{3} u_{6} \in K$.

The link of $u_{5}$ shows that $u_{5} u_{6}$ is an edge in one of $u_{2} u_{5} u_{6}, u_{3} u_{5} u_{6}$ or $u_{5} u_{6} u_{9}$.

If $u_{3} u_{5} u_{6} \in K$, considering the links of $u_{6}$ and $u_{1}, u_{1} u_{6} u_{8}$ and $u_{1} u_{3} u_{7}$ are faces. The links of $u_{5}, u_{7}$ and Claim 4 show that $u_{2} u_{3} u_{5} \notin K$. Hence, $\operatorname{Lk}\left(u_{5}\right)$ and $\operatorname{Lk}\left(u_{3}\right)$ show that $u_{3} u_{5} u_{9}$ and $u_{2} u_{5} u_{7} \in K$. The links of $u_{8}, u_{5}$ and Claim 4 show that $u_{5} u_{8} u_{9} \notin K$, $u_{5} u_{7} u_{9}$ and $u_{2} u_{5} u_{8} \in K$. Since $u_{6} u_{9} \notin \operatorname{Lk}\left(u_{3}\right)$, it is clear from $\operatorname{Lk}\left(u_{9}\right)$ and Claim 4 that $u_{2} u_{6} u_{9}$ and $u_{4} u_{6} u_{9}$ are faces. Since either $u_{2} u_{3} u_{9}$ or $u_{3} u_{4} u_{9}$ is a face, $\operatorname{Lk}\left(u_{3}\right)$ implies that $u_{3} u_{7} u_{8} \in K$. The links of $u_{7}, u_{6}$ and $u_{4}$ show that $u_{2} u_{4} u_{7}, u_{4} u_{6} u_{7}, u_{2} u_{6} u_{8}, u_{4} u_{8} u_{9}$ and $u_{3} u_{4} u_{8}$ are faces, thereby showing that $C_{5}\left(u_{3}, u_{4}, u_{9}, u, u_{7}\right)$ is in $\operatorname{Lk}\left(u_{8}\right)$. Therefore, either $u_{2} u_{5} u_{6}$ or $u_{5} u_{6} u_{9}$ is a face.

Subcase A: $u_{2} u_{5} u_{6} \in K$. The links of $u_{5}$ and $u_{7}$ and Claim 4 show that $u_{2} u_{3} u_{5} \notin K$. 
If $u_{2} u_{5} u_{9} \in K$, so is $u_{3} u_{5} u_{7}$. If $u_{5} u_{7} u_{8} \in K, \operatorname{Lk}\left(u_{7}\right), \operatorname{Lk}\left(u_{8}\right)$ and Claim 4 show that neither $u_{1} u_{6} u_{7}$ nor $u_{1} u_{6} u_{8}$ is a face, a contradiction. Hence, from $\operatorname{Lk}\left(u_{5}\right), u_{3} u_{5} u_{8}$ and $u_{5} u_{7} u_{9} \in K$. Considering the links of $u_{1}$ and $u_{7}$ we see that $u_{1} u_{6} u_{7}$ and $u_{1} u_{3} u_{8}$ are faces. This is seen to be impossible by applying Claim 4 to $\operatorname{Lk}\left(u_{7}\right)$. Hence, $\operatorname{Lk}\left(u_{5}\right)$ shows that $u_{2} u_{5} u_{7} \in K$.

We now observe, from $\operatorname{Lk}\left(u_{5}\right)$, that either $u_{3} u_{5} u_{7}$ or $u_{5} u_{7} u_{9}$ is a face.

In the first case the links of $u_{5}, u_{8}$ and $u_{1}$ show that $u_{5} u_{8} u_{9}, u_{3} u_{5} u_{9}, u_{1} u_{3} u_{8}$ and $u_{1} u_{6} u_{7}$ are faces (if $u_{1} u_{6} u_{8} \in K$, the remaining three vertices in $\operatorname{Lk}\left(u_{8}\right)$ are $u_{2}, u_{3}$ and $\left.u_{4}\right)$. Since $u_{6} u_{8} \notin \operatorname{Lk}\left(u_{3}\right), \operatorname{Lk}\left(u_{8}\right)$ shows that $u_{2} u_{6} u_{8}$ and $u_{4} u_{6} u_{8}$ are faces. The links of $u_{6}, u_{9}, u_{7}$ and $u_{8}$ show that $u_{3} u_{6} u_{9}, u_{4} u_{6} u_{9}, u_{2} u_{4} u_{9}, u_{2} u_{7} u_{9}, u_{3} u_{4} u_{7}, u_{4} u_{7} u_{8}$ and $u_{2} u_{3} u_{8}$ are faces and hence $K$ is $N_{10}$.

In the second case the links of $u_{5}, u_{8}$ and $u_{1}$ show that $u_{3} u_{5} u_{8}, u_{3} u_{5} u_{9}, u_{1} u_{6} u_{8}$ and $u_{1} u_{3} u_{7}$ are faces. It is easy to see from $\operatorname{Lk}\left(u_{3}\right), \operatorname{Lk}\left(u_{6}\right)$ and $\operatorname{Lk}\left(u_{9}\right)$ that $u_{4} u_{6} u_{7}$ and $u_{3} u_{6} u_{9}$ are faces (if either $u_{2} u_{3} u_{9}$ or $u_{3} u_{4} u_{9}$ is a face, then from Claim 4 and on completing $\operatorname{Lk}\left(u_{9}\right)$, we get $\left.\operatorname{deg}\left(u_{6}\right)<9\right)$. The links of $u_{9}, u_{4}, u_{6}, u_{7}$ and $u_{8}$ show that $u_{2} u_{4} u_{9}$, $u_{2} u_{6} u_{9}, u_{4} u_{8} u_{9}, u_{4} u_{6} u_{8}, u_{3} u_{4} u_{7}, u_{2} u_{7} u_{8}$, and $u_{2} u_{3} u_{8}$ are faces. Here, $K$ is $N_{4}$.

Subcase B: $u_{5} u_{6} u_{9}$ be a face. The link of $u_{9}$ and Claim 4 show that $u_{5} u_{7} u_{9} \notin K$ and hence $\operatorname{Lk}\left(u_{5}\right)$ shows that either $u_{2} u_{5} u_{9}$ or $u_{3} u_{5} u_{9}$ is a face.

B.1: $u_{2} u_{5} u_{9} \in K$. Here, $u_{3} u_{7} \in \operatorname{Lk}\left(u_{5}\right)$ and the links of $u_{5}, u_{7}, u_{8}$ and Claim 4 show that $u_{5} u_{7} u_{8} \notin K$. Now, $\operatorname{Lk}\left(u_{5}\right), \operatorname{Lk}\left(u_{8}\right)$ and $\operatorname{Lk}\left(u_{1}\right)$ show that $u_{3} u_{5} u_{8}, u_{2} u_{5} u_{7}, u_{1} u_{6} u_{8}$ and $u_{1} u_{3} u_{7} \in K$. Now, the links of $u_{7}$ and $u_{6}$ show that the edge $u_{7} u_{9}$ belongs to $u_{4} u_{7} u_{9}$ and therefore $u_{2} u_{6} u_{7}$ and $u_{4} u_{7} u_{8}$ are faces (if $u_{4} u_{6} u_{7} \in K$, then considering $\operatorname{Lk}\left(u_{4}\right)$, $\operatorname{Lk}\left(u_{9}\right)$ and $\operatorname{Lk}\left(u_{6}\right), u_{4} u_{6} u_{8}$ and $u_{2} u_{6} u_{9}$ are faces which show that $C_{3}\left(u_{2}, u_{5}, u_{6}\right)$ is in $\left.\operatorname{Lk}\left(u_{9}\right)\right)$. To complete $\operatorname{Lk}\left(u_{6}\right)$, either $u_{2} u_{4} u_{6}$ or $u_{4} u_{6} u_{9} \in K$.

In the first case the links of $u_{2}, u_{3}$ and $u_{8}$ show that $u_{2} u_{3} u_{8}, u_{2} u_{8} u_{9}, u_{3} u_{6} u_{9}, u_{3} u_{4} u_{9}$ and $u_{4} u_{6} u_{8}$ are faces. In this case, $K$ is $N_{13}$.

In the second case the links of $u_{9}, u_{8}$ and $u_{6}$ show that $u_{3} u_{8} u_{9}, u_{2} u_{3} u_{9}, u_{2} u_{4} u_{8}, u_{2} u_{6} u_{8}$ and $u_{3} u_{4} u_{6} \in K$. Here, $K$ is isomorphic, via the map $(0,1,5,7)(2,8)(3,6,9,4)$, to $N_{13}$.

B.2: $u_{3} u_{5} u_{9} \in K$. It is clear that if $u_{2} u_{3} u_{5}$ and $u_{5} u_{7} u_{8} \in K$, then the links of $u_{2}, u_{7}$, $u_{1}$ and $u_{3}$ show that $C_{7}\left(u_{3}, u_{6}, u_{1}, u_{5}, u_{7}, u, u_{9}\right)$ is in $\operatorname{Lk}\left(u_{8}\right)$. Therefore, from $\operatorname{Lk}\left(u_{5}\right)$, $u_{2} u_{5} u_{8}, u_{2} u_{5} u_{7}$ and $u_{3} u_{5} u_{7} \in K$. Now, if $u_{3} u_{7} u_{8} \in K$, the links of $u_{7}, u_{1}, u_{4}$ and $u_{8}$ show that $C_{5}\left(u_{4}, u_{8}, u, u_{1}, u_{7}\right)$ is in $\operatorname{Lk}\left(u_{9}\right)$. Hence, $u_{4} u_{7} u_{8}$ is a face.

In case $u_{4} u_{7} u_{9} \in K$, the links of $u_{7}, u_{1}, u_{6}$ and $u_{2}$ show that $u_{1} u_{3} u_{7}, u_{2} u_{6} u_{7}$, $u_{1} u_{6} u_{8} \in K$ and $u_{2} u_{6} u_{8}, u_{2} u_{3} u_{6} \notin K$ (if $u_{2} u_{3} u_{6} \in K$, to complete $\operatorname{Lk}\left(u_{6}\right), u_{4} u_{6} u_{8}$ and $u_{4} u_{6} u_{9} \in K$ which implies that $C_{4}\left(u_{6}, u_{9}, u_{7}, u_{8}\right)$ is in $\left.\operatorname{Lk}\left(u_{4}\right)\right)$. Since, $u_{3} u_{6} u_{9} \notin K$ (from $\operatorname{Lk}\left(u_{9}\right)$ ), to complete $\operatorname{Lk}\left(u_{6}\right), u_{2} u_{4} u_{6}$ and $u_{3} u_{4} u_{6} \in K$, a contradiction. Further, $u_{2} u_{4} u_{7} \notin K$ (if $u_{2} u_{4} u_{7} \in K$, completing $\operatorname{Lk}\left(u_{7}\right)$, we get $u_{2} u_{4} u_{9} \in K$, a contradiction). Hence, from $\operatorname{Lk}\left(u_{7}\right), u_{3} u_{4} u_{7} \in K$.

The links of $u_{7}, u_{1}, u_{4}, u_{6}$ and $u_{9}$ show that $u_{1} u_{6} u_{7}, u_{2} u_{7} u_{9}, u_{1} u_{3} u_{8}, u_{4} u_{6} u_{8}, u_{4} u_{6} u_{9}$, $u_{2} u_{4} u_{9}, u_{2} u_{3} u_{6}, u_{2} u_{6} u_{8}$ and $u_{3} u_{8} u_{9}$ are faces. In this case, $K$ is isomorphic, via the map $(2,9)(3,8)(4,7)(5,6)$, to $N_{10}$.

2.1.3: $u_{1} u_{4} u_{6} \in K$. Using the same method as the one above, we find that $K$ is isomorphic to one of $N_{2}, N_{4}, N_{5}, N_{7}, \ldots, N_{12}$.

2.1.4: $u_{1} u_{4} u_{8} \in K$. In this case we find that $K$ is isomorphic to one of $N_{4}, \ldots, N_{7}$, $N_{9}, \ldots, N_{14}$. 
Subcase 2.2: $u_{1} u_{3} u_{9} \in K$. The link of $u_{1}$ shows that one of $u_{1} u_{4} u_{6}, u_{1} u_{4} u_{5}, u_{1} u_{4} u_{7}$ or $u_{1} u_{4} u_{8} \in K$.

2.2.1: $u_{1} u_{4} u_{6} \in K$. We observe that either $u_{7} u_{8} \in \operatorname{Lk}\left(u_{1}\right)$ or $u_{7} u_{8} \notin \operatorname{Lk}\left(u_{1}\right)$

2.2.1.1: $u_{7} u_{8} \in \operatorname{Lk}\left(u_{1}\right)$. The link of $u_{1}$ shows that either $u_{1} u_{5} u_{7}$ or $u_{1} u_{5} u_{8} \in K$.

2.2.1.1.1: $u_{1} u_{5} u_{7} \in K$. In this case, $K$ is isomorphic to one of $N_{1}, N_{7}, N_{11}$ or $N_{14}$.

2.2.1.1.2: $u_{1} u_{5} u_{8} \in K$. The links of $u_{1}$ and $u_{7}$ show that $u_{1} u_{5} u_{6}$ and $u_{1} u_{3} u_{7} \in K$. Considering $\operatorname{Lk}\left(u_{6}\right)$ and $\operatorname{Lk}\left(u_{7}\right)$, we see that either $u_{2} u_{6} u_{7}$ or $u_{6} u_{7} u_{9} \in K$.

Claim 6. $u_{6} u_{7} u_{9}$ is a face.

If $u_{2} u_{6} u_{7} \in K$, then $\operatorname{Lk}\left(u_{7}\right)$ and $\operatorname{Lk}\left(u_{3}\right)$ shows that either $u_{3} u_{4} u_{7}$ or $u_{3} u_{5} u_{7} \in K$.

If $u_{3} u_{4} u_{7} \in K$, the links of $u_{7}, u_{4}, u_{5}$ and $u_{3}$ show that $u_{2} u_{5} u_{7}, u_{5} u_{7} u_{9}, u_{4} u_{7} u_{9}$, $u_{2} u_{4} u_{5}, u_{4} u_{6} u_{8}, u_{4} u_{8} u_{9}, u_{3} u_{5} u_{8}, u_{3} u_{5} u_{9}, u_{2} u_{3} u_{6}$ and $u_{3} u_{6} u_{8} \in K$. Here, $\operatorname{deg}\left(u_{6}\right)<9$. Hence, $u_{3} u_{5} u_{7} \in K$.

To complete $\operatorname{Lk}\left(u_{7}\right), u_{2} u_{4} u_{7}, u_{4} u_{7} u_{9}$ and $u_{5} u_{7} u_{9}$ have to be faces (if $u_{4} u_{5} u_{7} \in K$, $\operatorname{Lk}\left(u_{5}\right)$ implies that either $u_{3} u_{5} u_{9}$ or $u_{5} u_{8} u_{9}$ is a face, which is seen to be impossible from the links of $u_{3}$ and $u_{8}$ ). This shows that the face $\left(\neq u u_{4} u_{5}\right)$ having $u_{4} u_{5}$ as an edge is $u_{2} u_{4} u_{5}$, a contradiction. This proves Claim 6.

From $\operatorname{Lk}\left(u_{7}\right)$ and Claim 6 , we see that one of $u_{2} u_{3} u_{7}, u_{3} u_{4} u_{7}$ or $u_{3} u_{5} u_{7}$ is a face.

If $u_{3} u_{4} u_{7}$ is a face, the links of $u_{7}, u_{4}, u_{8}$ and $u_{5}$ show that $u_{2} u_{4} u_{7}, u_{2} u_{5} u_{7}, u_{5} u_{7} u_{9}$, $u_{4} u_{5} u_{9}$ and $u_{2} u_{3} u_{5}$ are faces which implies that $\operatorname{deg}\left(u_{2}\right)<9$.

If $u_{2} u_{3} u_{7}$ is a face, the links of $u_{7}, u_{2}, u_{5}, u_{3}$ and $u_{6}$ show that $u_{2} u_{5} u_{7}, u_{4} u_{5} u_{7}, u_{4} u_{7} u_{9}$, $u_{2} u_{5} u_{9}, u_{3} u_{5} u_{9}, u_{3} u_{5} u_{8}, u_{3} u_{4} u_{6}, u_{3} u_{6} u_{8}, u_{2} u_{6} u_{8}, u_{2} u_{6} u_{9}, u_{2} u_{4} u_{8}$ and $u_{4} u_{8} u_{9}$ are faces. Here, $K$ is $N_{3}$.

In the last case the link of $u_{7}$ shows that $u_{2} u_{4} u_{7}, u_{2} u_{5} u_{7}$ and $u_{4} u_{7} u_{9} \in K$ (if $u_{4} u_{5} u_{7} \in$ $K$, to complete $\operatorname{Lk}\left(u_{5}\right)$, either $u_{3} u_{5} u_{9}$ or $u_{5} u_{8} u_{9} \in K$, a contradiction). It is clear that $u_{4} u_{5} u_{9} \in K$, from $\operatorname{Lk}\left(u_{4}\right)$ and $\operatorname{Lk}\left(u_{5}\right)$. The links of $u_{4}, u_{6}, u_{2}$ and $u_{3}$ show that $u_{3} u_{4} u_{8}$, $u_{4} u_{6} u_{8}, u_{2} u_{3} u_{6}, u_{2} u_{6} u_{8}, u_{3} u_{6} u_{9}, u_{2} u_{8} u_{9}, u_{2} u_{5} u_{9}$ and $u_{3} u_{5} u_{8}$ are faces. Here, $K$ is isomorphic, via the map $(0,9,7)(1,2,5,4,6,8)$, to $N_{5}$.

2.2.1.2: $u_{7} u_{8} \notin \operatorname{Lk}\left(u_{1}\right)$. Hence, $u_{1} u_{5} u_{7}$ and $u_{1} u_{5} u_{8}$ are faces. To complete $\operatorname{Lk}\left(u_{1}\right)$, either $u_{1} u_{6} u_{7}$ and $u_{1} u_{3} u_{8}$ or $u_{1} u_{6} u_{8}$ and $u_{1} u_{3} u_{7}$ are faces

2.2.1.2.1: $u_{1} u_{6} u_{7}$ and $u_{1} u_{3} u_{8}$ are faces. The edge $u_{7} u_{8}$ belongs to one of $u_{2} u_{7} u_{8}$, $u_{3} u_{7} u_{8}$ or $u_{4} u_{7} u_{8}$.

Subcase A: $u_{2} u_{7} u_{8} \in K$. If $u_{2} u_{3} u_{7} \in K$, the links of $u_{7}$ and $u_{3}$ show that $u_{3} u_{7} u_{9}$, $u_{4} u_{7} u_{9}, u_{4} u_{5} u_{7}$ and $u_{3} u_{5} u_{8}$ are faces. This implies that $C_{3}\left(u_{5}, u_{1}, u_{3}\right)$ is in $\operatorname{Lk}\left(u_{8}\right)$. Hence, either $u_{2} u_{4} u_{7}$ or $u_{2} u_{7} u_{9} \in K$.

A.1: $u_{2} u_{7} u_{9} \in K$. To complete $\operatorname{Lk}\left(u_{7}\right), u_{3} u_{5} u_{7}, u_{3} u_{4} u_{7}$ and $u_{4} u_{7} u_{9}$ have to be faces. Considering $\operatorname{Lk}\left(u_{4}\right)$, we see that either $u_{4} u_{6} u_{9}$ or $u_{4} u_{8} u_{9} \in K$ (since, $\left.u_{2} u_{4} \notin \operatorname{Lk}\left(u_{9}\right)\right)$.

In the first case the links of $u_{4}, u_{8}, u_{9}$ and $u_{6}$ show that $u_{2} u_{4} u_{8}, u_{4} u_{5} u_{8}, u_{3} u_{6} u_{8}$, $u_{6} u_{8} u_{9}, u_{2} u_{5} u_{9}, u_{3} u_{5} u_{9}, u_{2} u_{3} u_{6}$ and $u_{2} u_{5} u_{6}$ are faces and, hence, $K$ is isomorphic, via the map $(0,3,1,2,8,4,6,5,9)$, to $N_{10}$.

In the second case the links of $u_{4}, u_{6}, u_{8}, u_{2}$ and $u_{9}$ show that $u_{4} u_{6} u_{8}, u_{2} u_{4} u_{5}, u_{2} u_{5} u_{8}$, $u_{3} u_{6} u_{8}, u_{2} u_{3} u_{6}, u_{2} u_{6} u_{9}, u_{5} u_{6} u_{9}$ and $u_{3} u_{5} u_{9}$ are faces. Here, $K$ is $N_{9}$.

A.2: $u_{2} u_{4} u_{7} \in K$. The link of $\left(u_{7}\right)$ shows that $u_{3} u_{7} u_{9} \in K$. Considering the links of $u_{8}$ and $u_{9}, u_{2} u_{8} u_{9}$ and $u_{2} u_{3} u_{9} \notin K$ and hence the link of $u_{2}$ shows that $u_{2} u_{5} u_{9}$ and $u_{2} u_{6} u_{9}$ are faces. To complete $\operatorname{Lk}\left(u_{7}\right)$, either $u_{3} u_{4} u_{7}$ or $u_{4} u_{7} u_{9} \in K$. 
In the first case the links of $u_{7}, u_{9}, u_{4}, u_{8}$ and $u_{5}$ show that $u_{5} u_{7} u_{9}, u_{4} u_{6} u_{9}, u_{4} u_{8} u_{9}$, $u_{4} u_{5} u_{8}, u_{2} u_{6} u_{8}, u_{3} u_{6} u_{8}, u_{2} u_{3} u_{5}$ and $u_{3} u_{5} u_{6}$ are faces. Now, $K$ is isomorphic, via the map $(0,2)(1,3)(5,9,8,6,7)$, to $N_{10}$.

In the second case $\operatorname{Lk}\left(u_{4}\right)$ shows that $u_{4} u_{6} u_{9} \notin K$. The links of $u_{7}, u_{9}, u_{4}, u_{8}$ and $u_{6}$ now show that $u_{3} u_{5} u_{7}, u_{4} u_{5} u_{9}, u_{6} u_{8} u_{9}, u_{2} u_{5} u_{8}, u_{2} u_{3} u_{6}, u_{3} u_{4} u_{8}, u_{4} u_{6} u_{8}$ and $u_{3} u_{5} u_{6}$ are faces. Here, $K$ is isomorphic, via the map $(0,4,5,1,7,9,8,6,2,3)$, to $N_{10}$.

Subcase B: $u_{3} u_{7} u_{8} \in K$. If $u_{2} u_{3} u_{7} \in K$, the links of $u_{7}, u_{2}$ and $u_{3}$ show that $u_{2} u_{7} u_{9}$, $u_{4} u_{7} u_{9}, u_{4} u_{5} u_{7}, u_{3} u_{5} u_{6}, u_{3} u_{5} u_{9}$ and $u_{3} u_{4} u_{6}$ are faces. This implies that $C_{6}\left(u_{3}, u_{4}, u_{1}, u_{7}\right.$, $\left.u, u_{5}\right)$ is in $\operatorname{Lk}\left(u_{6}\right)$. It is easy to see that $C_{4}\left(u_{7}, u_{8}, u_{1}, u_{9}\right)$ is in $\operatorname{Lk}\left(u_{3}\right)$ if $u_{3} u_{7} u_{9} \in K$. Hence, from $\operatorname{Lk}\left(u_{7}\right), u_{3} u_{4} u_{7}$ and $u_{2} u_{7} u_{9}$ are faces.

The link of $u_{3}$ shows that $u_{3} u_{5} u_{6}, u_{2} u_{3} u_{5}$ and $u_{3} u_{6} u_{9} \in K$. (If $u_{2} u_{3} u_{6}$ and $u_{3} u_{5} u_{9} \in$ $K$, the links of $u_{6}, u_{8}, u_{7}$ and $u_{2}$ show that $u_{6} u_{8} u_{9}, u_{2} u_{4} u_{8}, u_{2} u_{5} u_{7}, u_{4} u_{7} u_{9}, u_{2} u_{5} u_{8}$ and $u_{4} u_{6} u_{8} \in K$. Here, $C_{4}\left(u_{8}, u_{2}, u_{1}, u_{6}\right)$ is in $\operatorname{Lk}\left(u_{4}\right)$.) The links of $u_{6}, u_{9}, u_{7}$, $u_{4}$ and $u_{8}$ show that $u_{4} u_{6} u_{8}, u_{2} u_{6} u_{8}, u_{2} u_{6} u_{9}, u_{4} u_{5} u_{9}, u_{4} u_{8} u_{9}, u_{5} u_{7} u_{9}, u_{2} u_{4} u_{7}$ and $u_{2} u_{5} u_{8}$ are faces. Here, $K$ is isomorphic, via the map $(0,9,5,2)(1,8,3)(4,7,6)$, to $N_{10}$.

Subcase C: $u_{4} u_{7} u_{8} \in K . \operatorname{Lk}\left(u_{7}\right)$ shows that one of $u_{2} u_{4} u_{7}, u_{3} u_{4} u_{7}$ or $u_{4} u_{7} u_{9}$ is a face.

C.1: $u_{2} u_{4} u_{7} \in K$. The links of $u_{2}$ and $u_{7}$ show that $u_{2} u_{7} u_{9}, u_{3} u_{7} u_{9}$ and $u_{3} u_{5} u_{9} \in K$. Since $u_{4} u_{9} \notin \operatorname{Lk}\left(u_{2}\right)$ and $\operatorname{Lk}\left(u_{9}\right)$, the links of $u_{9}, u_{4}, u_{3}, u_{6}$ and $u_{5}$ show that $u_{4} u_{5} u_{9}$, $u_{4} u_{6} u_{9}, u_{3} u_{4} u_{8}, u_{2} u_{3} u_{6}, u_{3} u_{5} u_{6}, u_{2} u_{6} u_{8}, u_{6} u_{8} u_{9}, u_{2} u_{5} u_{8}$ and $u_{2} u_{5} u_{9}$ are faces. In this case, $K$ is isomorphic, via the map $(0,7,2,8,5,1,4,6,9,3)$, to $N_{10}$.

C.2: $u_{3} u_{4} u_{7} \in K$. It is easy to see from $\operatorname{Lk}\left(u_{4}\right)$ that $u_{4} u_{5} u_{9} \in K$ (since $u_{4} u_{5} u_{8} \notin K$ from $\left.\operatorname{Lk}\left(u_{8}\right)\right)$. The links of $u_{7}, u_{3}, u_{8}, u_{5}$ and $u_{6}$ show that $u_{2} u_{5} u_{7}, u_{2} u_{7} u_{9}, u_{3} u_{7} u_{9}$, $u_{2} u_{3} u_{5}, u_{3} u_{5} u_{6}, u_{3} u_{6} u_{8}, u_{5} u_{8} u_{9}, u_{2} u_{6} u_{8}, u_{2} u_{4} u_{8}, u_{2} u_{6} u_{9}$ and $u_{4} u_{6} u_{9}$ are faces. In this case, $K$ is isomorphic, via the map $(0,5,3,1,2,8)(4,7,6,9)$, to $N_{9}$.

C.3: $u_{4} u_{7} u_{9} \in K$. The link of $\left(u_{7}\right)$ shows that $u_{3} u_{5} u_{7}, u_{2} u_{3} u_{7}$ and $u_{2} u_{7} u_{9} \in K$ (if $u_{3} u_{7} u_{9} \in K$, the links of $u_{7}, u_{3}$ and $u_{8}$ show that $\left.u_{3} u_{4} u_{5} \in K\right)$. Since $u_{3} u_{5} \notin \operatorname{Lk}\left(u_{8}\right)$, either $u_{3} u_{5} u_{6}$ or $u_{3} u_{5} u_{9} \in K$. If $u_{3} u_{5} u_{6} \in K$, the links of $u_{5}$ and $u_{2}$ show that $u_{2} u_{4} u_{6} \in$ $K$, which implies that $C_{3}\left(u_{2}, u_{1}, u_{6}\right)$ is in $\operatorname{Lk}\left(u_{4}\right)$. Hence, $u_{3} u_{5} u_{9} \in K$.

The links of $u_{3}, u_{2}, u_{4}, u_{5}$ and $u_{6}$ show that $u_{3} u_{4} u_{6}, u_{3} u_{6} u_{8}, u_{2} u_{4} u_{8}, u_{4} u_{5} u_{9}, u_{2} u_{5} u_{6}$, $u_{2} u_{5} u_{8}, u_{2} u_{6} u_{9}$ and $u_{6} u_{8} u_{9} \in K$. Now, $K$ is isomorphic, via the map $(0,8,7,4,3,1,9)$ $(2,5)$, to $N_{10}$.

2.2.1.2.2: $u_{1} u_{6} u_{8}$ and $u_{1} u_{3} u_{7}$ are faces. In this case, using the above method, $K$ is isomorphic to one of $N_{5}, N_{6}, N_{7}, N_{9}, N_{10}$ or $N_{12}$.

2.2.2: $u_{1} u_{4} u_{5} \in K$. In this case, $K$ is isomorphic to $N_{11}$.

2.2.3: $u_{1} u_{4} u_{7} \in K$. In this case, $K$ is isomorphic to $N_{4}, N_{5}, N_{7}, N_{10}, \ldots, N_{12}$ or $N_{14}$.

2.2.4: $u_{1} u_{4} u_{8} \in K$. In this case, $K$ is isomorphic to one of $N_{4}, N_{7}, N_{10}, N_{11}$ or $N_{14}$.

Subcase 2.3: $u_{1} u_{5} u_{9} \in K$. In this case, $K$ is isomorphic to one of $N_{1}, N_{4}, \ldots, N_{14}$.

Subcase 2.4: $u_{1} u_{6} u_{9} \in K$. In this case, $K$ is isomorphic to one of $N_{1}, N_{3}, \ldots, N_{14}$.

The theorem now follows from Lemma 5.

Proof of Theorem 7. Let $K$ be an $n$-vertex $(n \leq 11)\{3, q\}$-equivelar polyhedron. If $\chi(K)>0$, then, by Corollary $3, K$ is isomorphic to $S_{4}^{2}, O$ or $\mathbb{R} P_{6}^{2}$. 
If $\chi(K) \leq 0$, then, by (2), $q \geq 6$ and hence $n \geq 7$. From (1), $n q$ is divisible by 6 . So, $(n, q)=(7,6),(8,6),(9,6),(9,8),(10,6),(10,9)$ or $(11,6)$.

If $q=6$, then $\chi(K)=0$ and hence, by Theorem $4, K$ is isomorphic to $T_{7}, \ldots, T_{11}$, $A_{3,3}, B_{3,3}$ or $Q$. Since, the non-edge graphs of $T_{9}, A_{3,3}$ and $B_{3,3}$ are pairwise nonisomorphic, $T_{9}, A_{3,3}$ and $B_{3,3}$ are pairwise non-isomorphic. Observe that $\operatorname{NEG}(Q)$ is a bipartite graph. As $\operatorname{NEG}\left(T_{10}\right)$ contains an induced pentagon, it is therefore not isomorphic to $\operatorname{NEG}(Q)$. Hence $Q \neq T_{10}$. Thus, all these eight polyhedra are distinct (non-isomorphic).

If $(n, q)=(9,8)$, then, by Theorem $5, K$ is isomorphic to $M_{1}$ or $M_{2}$. Moreover, $M_{1} ¥ M_{2}$.

If $(n, q)=(10,9)$, then, by Theorem $6, K$ is isomorphic to $N_{1}, \ldots, N_{14}$. Moreover, $N_{i} \not N_{j}$ for $1 \leq i \neq j \leq 14$. This completes the proof.

\section{Acknowledgments}

The authors thank Bhaskar Bagchi for the idea to calculate the characteristic polynomials of adjacency matrices to prove Lemmas 4 and 5 and for making them aware of several important papers in the literature related to this work. The authors are thankful to C. R. Pranesachar for useful conversations. Finally, the authors thank an anonymous referee for many useful comments which led to substantial improvements in the presentation of this paper and for making them aware of several important papers in the literature related to this work. Remark 4 is also due to the referee.

\section{References}

[1] B. Bagchi and B. Datta, A structure theorem for pseudomanifolds, Discrete Math. 168 (1998), 41-60.

[2] J. A. Bondy and U. S. R. Murty, Graph Theory with Applications, Elsevier North-Holland, Amsterdam, 1976.

[3] U. Brehm, Polyhedral maps with few edges, in Topics in Combinatorics and Graph Theory (RingelFetstschrift) (eds. R. Bodendiek and R. Henn), Physica-Verlag, Heidelberg, 1990, pp. 153-162.

[4] U. Brehm and E. Schulte, Polyhedral maps, in Handbooks of Discrete and Computational Geometry (eds. J. E. Goodman and J. O’Rourke), CRC Press, Boca Raton, FL, 1997, pp. 345-358.

[5] U. Brehm and J. M. Wills, Polyhedral manifolds, in Handbook of Convex Geometry (eds. P. M. Gruber and J. M. Wills), Elsevier, Dordrecht, 1993, pp. 535-554.

[6] H. S. M. Coxeter, Regular Polytopes, Dover, New York, 1973.

[7] H. S. M. Coxeter and W. O. J. Moser, Generators and Relations for Discrete Groups, Springer-Verlag, New York, 1972.

[8] L. Danzer and E. Schulte, Reguläre Inzidenzkomplexe I, Geom. Dedicata 13 (1982), 295-308.

[9] B. Datta, Two dimensional weak pseudomanifolds on seven vertices, Bol. Soc. Mat. Mexicana 5 (1999), 419-426.

[10] B. Datta and N. Nilakantan, Two dimensional weak pseudomanifolds on 8 vertices (preprint).

[11] A. L. Edmonds, J. H. Ewing and R. S. Kulkarni, Regular tessellations of surfaces and $(p, q, 2)$-triangle groups, Ann. of Math. 116 (1982), 113-132.

[12] B. Grünbaum, Convex Polytopes, Interscience, New York, 1967.

[13] M. Jungerman and G. Ringel, Minimal triangulations on orientable surfaces, Acta Math. 145 (1980), $121-154$.

[14] W. Kühnel, Triangulations of manifolds with few vertices, in Advances in Differential Geometry and Topology (ed. F. Tricerri), World Scientific, Singapore, 1990, pp. 59-114. 
[15] W. Magnas, Noneuclidean Tesselations and Their Groups, Academic Press, New York, 1974.

[16] P. McMullen, B. Monson and A. Ivic Weiss, Regular maps constructed from linear groups, European J. Combin. 14 (1993), 541-552.

[17] P. McMullen and E. Schulz, Finite quotients of infinite universal polytopes, in Discrete and Computational Geometry (eds. J. Goodman, R. Pollack and W. Steiger), DIMACS Series in Discrete Mathematics and Theoretical Computer Science, vol. 6, American Mathematical Society, Providence, RI, 1991, pp. 231236.

[18] P. McMullen, Ch. Schulz and J. M. Wills, Equivelar polyhedral manifolds in $E^{3}$, Israel J. Math. 41 (1982), 331-346.

[19] P. McMullen, Ch. Schulz and J. M. Wills, Polyhedral 2-manifolds in $E^{3}$ with unusually large genus, Israel J. Math. 46 (1983), 127-144.

[20] P. McMullen, Ch. Schulz and J. M. Wills, Two remarks on equivelar manifolds, Israel J. Math. 52 (1985), $28-32$.

[21] G. Ringel, Bestimmung der Maximalzahl der Nachbargebiete auf nichtorientierbaren Flächen, Math. Ann. 127 (1954), 181-214.

[22] G. Ringel, Wie man die geschlossenen nichtorientierbaren Flächen in möglichst wenig Dreiecke zerlegen kann, Math. Ann. 130 (1955), 317-326.

[23] G. Ringel, Map Color Theorem, Springer-Verlag, Berlin, 1974.

[24] E. Schulte, Reguläre Inzidenzkomplexe II \& III, Geom. Dedicata 14 (1983), 33-56 \& 57-79.

[25] A. Vince, Regular combinatorial maps, J. Combin. Theory Ser. B 35 (1983), 256-277.

Received February 14, 2000, and in revised form August 15, 2000. Online publication March 26, 2001. 